\title{
An Order-Theoretic Property of the Commutator
}

\author{
Keith A. Kearnes
}

\begin{abstract}
We describe a new order-theoretic property of the commutator for finite algebras. As a corollary we show that any right nilpotent congruence on a finite algebra is left nilpotent. The result is false for infinite algebras and the converse is false even for finite algebras. We show further that any solvable $E$-minimal algebra is left nilpotent, any finite algebra whose congruence lattice contains a 0,1 -sublattice isomorphic to $\mathbf{M}_{\mathbf{3}}$ is left nilpotent and any homomorphic image of a finite abelian algebra is left and right nilpotent.
\end{abstract}

\section{Introduction}

The introduction of the commutator into universal algebra was an extraordinary boon to researchers in the field. Where previously the congruence lattice was one of the most important invariants associated with an algebra, one can now study the "commutation lattice" and extract even more information about the algebra. The commutator - a binary operation on the congruence lattice of an algebra - has found its deepest applications in the study of varieties whose algebras have modular congruence lattices. For algebras in congruence modular varieties one can prove that the commutator satisfies

$$
[\beta, \alpha]=[\alpha, \beta] \leq \alpha \wedge \beta
$$

(we say that the commutator is symmetric and submultiplicative) and

$$
\left[\bigvee_{i \in I} \alpha_{i}, \beta\right]=\bigvee_{i \in I}\left[\alpha_{i}, \beta\right]
$$

(the commutator is completely additive.) The theory of the modular commutator is developed in [2].

For varieties which are non-modular the commutator has been important, but less so, since it no longer has the nice properties described above. It is still a binary operation definable on the congruence lattice of any algebra which is submultiplicative, monotone in each variable and satisfies

$$
\left[\alpha_{i}, \beta\right]=\gamma \text { for all } i \in I \longrightarrow\left[\bigvee_{i \in I} \alpha_{i}, \beta\right]=\gamma
$$


(We say that the commutator is semidistributive with respect to arbitrary joins in its left variable.) We call these properties order-theoretic properties of the commutator because they express connections between the commutator operation and the order relation of the congruence lattice.

The loss of symmetry and additivity severely limits the commutator's usefulness for nonmodular varieties. However, in Chapter 7 of [4], D. Hobby and R. McKenzie show that for finite algebras and locally finite varieties the commutator has non-obvious properties which make the notion of solvability well-behaved. For example, it is a consequence of their work that homomorphic images of finite solvable algebras are solvable - a result which fails for infinite algebras. In [3], Freese, Kearnes and Nation refine Hobby and McKenzie's commutator result.

In this paper we exhibit another non-obvious property of the commutator for finite algebras. We begin by analyzing what it means for a congruence $\beta$ on a finite algebra $\mathbf{A}$ to "annihilate" a tame quotient $\langle\delta, \theta\rangle$ of $\mathbf{A}$. We compare the relationship between four possible interpretations of this sentence: the sentence may be interpreted to mean that $(i) C(\beta, \theta ; \delta)$ holds, $(i i)[\beta, \theta] \leq \delta$ holds, (iii) $C(\theta, \beta ; \delta)$ holds, or $(i v)[\theta, \beta] \leq \delta$ holds. Here $C(\alpha, \phi ; \psi)$ denotes the ternary centralizer relation. These four interpretations are distinct from each other, but the implications $(i) \rightarrow(i i)$ and $(i i i) \rightarrow(i v)$ follow immediately from the definitions of the commutator and the centralizer. Our main result is to show that, under certain additional hypotheses, other implications hold. As a consequence we are able to prove that the right nilpotent congruences on a finite algebra are left nilpotent (and we give examples to prove that this statement is false for infinite algebras and that the converse is false even for finite algebras). Other results which follow are: Every solvable E-minimal algebra is left nilpotent. Every finite algebra whose congruence lattice contains $\mathbf{M}_{\mathbf{3}}$ as a 0 ,1-sublattice is left nilpotent. Every homomorphic image of a finite, abelian algebra is left and right nilpotent.

Our reference for algebra is [6] and our reference for tame congruence theory is [4]. However, the next section summarizes most of what we use from [4].

\section{Tame Congruence Theory}

The set of $n$-ary polynomials of an algebra $\mathbf{A}$ will be denoted by $\operatorname{Pol}_{n} \mathbf{A}$ and the clone of all polynomials of $\mathbf{A}$ will be denoted $\mathrm{Pol} \mathbf{A}$. If $X$ is a subset of $A$, then $\left.(\mathrm{Pol} \mathbf{A})\right|_{X}$ denotes the set of all restrictions to $X$ of polynomials $f \in \operatorname{Pol}$ A which satisfy $f\left(X^{n}\right) \subseteq X$ for appropriate $n$. $\left.\mathbf{A}\right|_{X}$ denotes the non-indexed algebra $\left\langle X ;\left.(\operatorname{Pol} \mathbf{A})\right|_{X}\right\rangle$. The idempotent unary polynomials of $\mathbf{A}$ will be denoted by $E(\mathbf{A})$. If $\mathbf{A}$ is a finite algebra with congruences $\delta<\theta$, then $\mathbf{U}_{\mathbf{A}}(\delta, \theta)$ denotes the set of all sets of the form $f(A)$ for some $f \in \operatorname{Pol}_{1} \mathbf{A}$ which satisfies $f(\theta) \nsubseteq \delta \delta$ and $\mathrm{M}_{\mathbf{A}}(\delta, \theta)$ denotes the set of members of $\mathrm{U}_{\mathbf{A}}(\delta, \theta)$ which are minimal with respect to inclusion. The members of $\mathrm{M}_{\mathbf{A}}(\delta, \theta)$ are called the $\langle\delta, \theta\rangle$-minimal sets.

If $\delta<\theta$ in Con $\mathbf{A}$, then $\langle\delta, \theta\rangle$ is called a congruence quotient. If $\mathbf{A}$ is finite, $\langle\delta, \theta\rangle$ is called a tame quotient precisely when there is a $U \in \mathrm{M}_{\mathbf{A}}(\delta, \theta)$ and $e \in E(\mathbf{A})$ such that $(i)$ $e(A)=U$ and $(i i)$ whenever $\delta<\psi<\theta$ holds in Con $\mathbf{A}$ we have $\left.\delta\right|_{U}<\left.\psi\right|_{U}<\left.\theta\right|_{U}$. Here $\left.\right|_{U}$ denotes restriction to $U$. A congruence quotient $\langle\delta, \theta\rangle$ where $\delta \prec \theta$ is called a prime quotient. Prime quotients are tame. If $\langle\delta, \theta\rangle$ is tame and $U \in \mathrm{M}_{\mathbf{A}}(\delta, \theta)$, then any $\left.\theta\right|_{U^{-c l a s s}}$ 
of $U$ which is different from a $\left.\delta\right|_{U}$-class is called a trace of $U$ and also a $\langle\delta, \theta\rangle$-trace of $\mathbf{A}$. The union of the traces of $U$ is called the body of $U$. The rest of $U$ is called the tail.

If $\langle\delta, \theta\rangle$ is tame, then the members of $\mathrm{M}_{\mathbf{A}}(\delta, \theta)$ are polynomially isomorphic in the sense that if $U, V \in \mathrm{M}_{\mathbf{A}}(\delta, \theta)$, then there are polynomials $f, g \in \mathrm{Pol}_{1} \mathbf{A}$ such that $f(U)=V$, $g(V)=U,\left.g f\right|_{U}=\operatorname{id}_{U}$ and $\left.f g\right|_{V}=\operatorname{id}_{V}$.

If $(a, b) \in \theta-\delta$ and $U \in \mathrm{M}_{\mathbf{A}}(\delta, \theta)$, then there is an $f \in \mathrm{Pol}_{1} \mathbf{A}$ such that $f(A)=U$ and $(f(a), f(b)) \in \theta-\delta$. Further, there are elements $a=x_{0}, x_{1}, \ldots, x_{n}=b$ such that $\left(x_{i}, x_{i+1}\right) \in \delta$ when $i$ is even and $\left\{x_{i}, x_{i+1}\right\} \subseteq T_{i}$ for some $\langle\delta, \theta\rangle$-trace $T_{i}$ when $i$ is odd. We say that $a$ and $b$ are connected modulo $\delta$ by $\langle\delta, \theta\rangle$-traces.

The type of a tame quotient $\langle\delta, \theta\rangle$ depends on the structure of the non-indexed algebra $\left.\mathbf{A}\right|_{T} /\left.\delta\right|_{T}$ where $T$ is some (any) $\langle\delta, \theta\rangle$-trace of $\mathbf{A}$. We write $\operatorname{typ}(\delta, \theta)=\mathbf{i}$ where $\mathbf{i} \in\{\mathbf{1}, \mathbf{2}, \mathbf{3}, \mathbf{4}, \mathbf{5}\}$ according to the following rules:

$\mathbf{i}=\mathbf{1}$ if $\left.\mathbf{A}\right|_{T} /\left.\delta\right|_{T}$ is polynomially equivalent to a G-set;

$\mathbf{i}=\mathbf{2}$ if $\left.\mathbf{A}\right|_{T} /\left.\delta\right|_{T}$ is polynomially equivalent to a vector space;

$\mathbf{i}=\mathbf{3}$ if $\left.\mathbf{A}\right|_{T} /\left.\delta\right|_{T}$ is polynomially equivalent to a 2-element Boolean algebra;

$\mathbf{i}=4$ if $\left.\mathbf{A}\right|_{T} /\left.\delta\right|_{T}$ is polynomially equivalent to a 2-element lattice;

$\mathbf{i}=\mathbf{5}$ if $\left.\mathbf{A}\right|_{T} /\left.\delta\right|_{T}$ is polynomially equivalent to a 2-element semilattice.

These are the only possibilities. Further information about the structure of $\left.\mathbf{A}\right|_{U}$ for any $U \in \mathrm{M}_{\mathbf{A}}(\delta, \theta)$ can be found in Chapter 4 of [4]

Let $\mathbf{A}$ be an algebra and $R$ and $S$ be binary relations on $A$. We say that the $\langle R, S\rangle$-term condition holds if for all $n$ whenever $(a, b) \in R,\left(u_{i}, v_{i}\right) \in S, i<n$, and $p(x, \bar{y}) \in \operatorname{Pol}_{n+1} \mathbf{A}$ one has

$$
p(a, \bar{u})=p(a, \bar{v}) \longleftrightarrow p(b, \bar{u})=p(b, \bar{v}) .
$$

If $\gamma$ is a congruence on $\mathbf{A}$ and the $\langle R / \gamma, S / \gamma\rangle$-term condition holds in $\mathbf{A} / \gamma$ we say that $R$ centralizes $S$ modulo $\gamma$ and we write $C(R, S ; \gamma)$.

Observe that $C(R, S ; \gamma)$ holds if and only if $C(\alpha, \beta ; \gamma)$ holds, where $\alpha=\operatorname{Cg}^{\mathrm{A}}(R \cup \gamma)$ and $\beta$ is the reflexive, symmetric, compatible relation generated by $S$. For this paper we will only be interested in $C(\alpha, \beta ; \gamma)$ when $\alpha, \beta$ and $\gamma$ are congruences; but we will use the fact that if $C(\alpha, \beta ; \gamma)$ fails and $\alpha=\operatorname{Cg}^{\mathrm{A}}(R \cup \gamma)$ for some $R$, then $C(R, \beta ; \gamma)$ fails.

In some examples we will use the following equivalent condition for $C(\alpha, \beta ; \gamma)$. When $\alpha, \beta$ and $\gamma$ are congruences on $\mathbf{A}, C(\alpha, \beta ; \gamma)$ holds if and only if the following statement is true: if $\mathbf{B}$ is equal to the subalgebra of $\mathbf{A}^{2}$ whose universe is $\beta$ and $\delta$ is the congruence on B generated by the pairs $S=\{\langle(x, x),(y, y)\rangle \mid(x, y) \in \alpha\}$, then $\beta \wedge \gamma$, as a subset of $B$, is a union of $\delta$-classes. It suffices for $\beta \wedge \gamma$ to be a union of $\delta^{\prime}$-classes for any $\delta^{\prime} \in$ Con $\mathbf{B}$ which contains $S$.

For any congruences $\alpha, \beta \in \operatorname{Con} \mathbf{A}$ the set $\{\delta \in \operatorname{Con} \mathbf{A} \mid C(\alpha, \beta ; \delta)\}$ is closed under arbitrary intersection, so there is a least congruence $\delta$ such that $\alpha$ centralizes $\beta$ modulo $\delta$. We write $[\alpha, \beta]$ for this $\delta$. If

$$
[\alpha, \beta] \leq \delta<\theta \leq \alpha \wedge \beta
$$

and $\langle\delta, \theta\rangle$ is tame, then the type of $\langle\delta, \theta\rangle$ is either $\mathbf{1}$ or $\mathbf{2}$. 
We define

$$
(\alpha]^{1}=[\alpha)^{1}=[\alpha]^{1} \stackrel{\text { def }}{=} \alpha,(\alpha, \beta]^{1}=[\alpha, \beta)^{1} \stackrel{\text { def }}{=}[\alpha, \beta]
$$

and then set

1. $(\alpha]^{n+1}=\left[\alpha,(\alpha]^{n}\right]$,

2. $[\alpha)^{n+1}=\left[[\alpha)^{n}, \alpha\right]$,

3. $[\alpha]^{n+1}=\left[[\alpha]^{n},[\alpha]^{n}\right]$,

4. $(\alpha, \beta]^{n+1}=\left[\alpha,(\alpha, \beta]^{n}\right]$ and

5. $[\alpha, \beta)^{n+1}=\left[[\alpha, \beta)^{n}, \beta\right]$.

If $(\alpha]^{n+1}=0$ for some $n$ we will say that $\alpha$ is $n$-step left nilpotent. If $[\alpha)^{n+1}=0$ for some $n$ we will say that $\alpha$ is $n$-step right nilpotent. If $[\alpha]^{n+1}=0$ for some $n$ we will say that $\alpha$ is $n$-step solvable. If $\alpha$ and $\beta$ are congruences on a finite algebra, then we will write $\alpha \stackrel{s}{\sim} \beta$ if $[\alpha \vee \beta]^{n} \leq \alpha \wedge \beta$ for some $n$. (Our notation for nilpotence is taken from Definition 4.35 of [4]. Our definition of $\stackrel{s}{\sim}$ is not the same as Definition 7.3 of [4], but the definitions are equivalent for finite algebras as Corollary 7.5 of [4] proves.)

Theorem 2.1 (See Proposition 3.4 of [4]) For an algebra $\mathbf{A}$ and congruences $\alpha, \alpha_{i}, \ldots, \delta_{j}$ the following hold.

(i) If $C(\alpha, \beta ; \delta)$ and $\alpha^{\prime} \leq \alpha, \beta^{\prime} \leq \beta$, then $C\left(\alpha^{\prime}, \beta^{\prime} ; \delta\right)$.

(ii) If $C\left(\alpha_{i}, \beta ; \delta\right)$ for all $i \in I$, then $C\left(\bigvee_{i \in I} \alpha_{i}, \beta ; \delta\right)$.

(iii) If $C\left(\alpha, \beta ; \delta_{j}\right)$ for all $j \in J$, then $C\left(\alpha, \beta ; \bigwedge_{j \in J} \delta_{j}\right)$.

(iv) $C(\alpha, \beta ; \delta) \leftrightarrow C(\alpha, \beta ; \beta \wedge \delta)$.

(v) If $\beta \wedge(\alpha \vee(\beta \wedge \delta)) \leq \delta$, then $C(\alpha, \beta ; \delta)$.

Related to the term condition is the strong term condition. We say that $\theta$ is strongly abelian over $\delta$ if $\delta \leq \theta$ and for all $n$ and all $p(\bar{x}) \in \operatorname{Pol}_{n} \mathbf{A}, \bar{u}, \bar{v} \in A^{n}$ and $\bar{w} \in A^{n-1}$ with $u_{i} \theta v_{i} \theta w_{i}$ for $i<n-1$ and $u_{n-1} \theta v_{n-1}$ we have

$$
\begin{gathered}
p\left(u_{0}, \ldots, u_{n-2}, u_{n-1}\right) \delta p\left(v_{0}, \ldots, v_{n-2}, v_{n-1}\right) \longrightarrow \\
p\left(w_{0}, \ldots, w_{n-2}, u_{n-1}\right) \delta p\left(w_{0}, \ldots, w_{n-2}, v_{n-1}\right) .
\end{gathered}
$$

If $\langle\delta, \theta\rangle$ is a tame quotient of a finite algebra $\mathbf{A}$, then $\theta$ is strongly abelian over $\delta$ if and only if $\langle\delta, \theta\rangle$ is of type $\mathbf{1}$. When $\mathbf{A}$ is finite and $\alpha$ and $\beta$ are congruences on $\mathbf{A}$ we will write $\alpha \stackrel{s s}{\sim} \beta$ if there are congruences $\gamma_{i}, i<n$, such that $\alpha \wedge \beta=\gamma_{0} \leq \cdots \leq \gamma_{n-1}=\alpha \vee \beta$ and $\gamma_{i+1}$ is strongly abelian over $\gamma_{i}$ for each $i$.

All of the material of this section until this point can be found in the early chapters of [4]. Now we begin describing a ramification of the usual type labeling of tame congruence theory. 
In this paragraph, $\langle\delta, \theta\rangle$ will always denote a prime congruence quotient. As mentioned above, a prime quotient $\langle\delta, \theta\rangle$ of a finite algebra $\mathbf{A}$ is tame and may be assigned a type label from the set $\{\mathbf{1}, \mathbf{2}, \mathbf{3}, \mathbf{4}, \mathbf{5}\}$ depending on the structure of the algebra $\left.\mathbf{A}\right|_{T} /\left.\delta\right|_{T}$ where $T$ is a $\langle\delta, \theta\rangle$-trace. For the nonabelian types, $\mathbf{3}, \mathbf{4}$ and $\mathbf{5}$, the structure of $\left.\mathbf{A}\right|_{T} /\left.\delta\right|_{T}$ up to polynomial equivalence does not depend on $\mathbf{A}$ or $\langle\delta, \theta\rangle$. For the abelian types the situation is more complicated. If $\operatorname{typ}(\delta, \theta)=\mathbf{2}$, then $\left.\mathbf{A}\right|_{T} /\left.\delta\right|_{T}$ is polynomially equivalent to a 1-dimensional vector space over a finite field. The structure of $\left.\mathbf{A}\right|_{T} /\left.\delta\right|_{T}$ in this case is determined by specifying that $\operatorname{typ}(\delta, \theta)=\mathbf{2}$ and giving the cardinality of $T /\left.\delta\right|_{T}$ which equals the cardinality of the scalar field for $\left.\mathbf{A}\right|_{T} /\left.\delta\right|_{T}$. If $\operatorname{typ}(\delta, \theta)=\mathbf{2}$, we will say that the subtype of $\langle\delta, \theta\rangle$ is $q=\left|\left(T /\left.\delta\right|_{T}\right)\right|$. We suggest writing both $\operatorname{subtyp}(\delta, \theta)=\mathbf{q}$ and $\operatorname{typ}(\delta, \theta)=\mathbf{2}_{\mathbf{q}}$ to express this. If $\operatorname{typ}(\delta, \theta)=\mathbf{1}$, then $\left.\mathbf{A}\right|_{T} /\left.\delta\right|_{T}$ is polynomially equivalent to a simple faithful G-set. The structure of $\left.\mathbf{A}\right|_{T} /\left.\delta\right|_{T}$ in this case is determined by specifying $G$, the group of unary polynomial permutations of $\left.\mathbf{A}\right|_{T} /\left.\delta\right|_{T}$, and $H$, any one point stabilizer subgroup of $G$. We will write both $\operatorname{subtyp}(\delta, \theta)=(\mathbf{G}, \mathbf{H})$ and $\operatorname{typ}(\delta, \theta)=\mathbf{1}_{(\mathbf{G}, \mathbf{H})}$ to mean that $\left.\mathbf{A}\right|_{T} /\left.\delta\right|_{T}$ is polynomially equivalent to the G-set of left cosets, $G / H$, under left multiplication by elements of $G$. If $G$ is trivial, then $\left.\mathbf{A}\right|_{T} /\left.\delta\right|_{T}$ is polynomially equivalent to a 2-element set. Instead of writing $\operatorname{typ}(\delta, \theta)=$ $\mathbf{1}_{\left(\mathbf{z}_{1}, \mathbf{z}_{1}\right)}$ in this case we will follow the suggestion in [5] and say that $\operatorname{typ}(\delta, \theta)=\mathbf{0}$. If $H$ is trivial, then $G$ is cyclic of prime order or trivial; because when $G$ is not trivial, $H$ is a maximal subgroup of $G$. For brevity we will say that both $\left.\mathbf{A}\right|_{T} /\left.\delta\right|_{T}$ and $\langle\delta, \theta\rangle$ are cyclic in this case. $\left.\mathbf{A}\right|_{T} /\left.\delta\right|_{T}$ is cyclic exactly when the non-identity polynomial permutations of $\left.\mathbf{A}\right|_{T} /\left.\delta\right|_{T}$ have no fixed points.

Analogous to the idea of a cyclic prime quotient of type $\mathbf{1}$ is the idea of a regular tame quotient of type $\mathbf{1}$. First, if $R \subseteq A \times A, p(x, \bar{y}) \in \operatorname{Pol}_{n+1} \mathbf{A}$ and $\bar{a}, \bar{b} \in A^{n}$ with $\left(a_{i}, b_{i}\right) \in R$ for all $i$, then we will call the unary polynomials $p(x, \bar{a})$ and $p(x, \bar{b}) R$-twins. Now let $\langle\delta, \theta\rangle$ be a tame quotient of type $\mathbf{1}$. For each $\langle\delta, \theta\rangle$-trace $T$ let $H_{T, R}$ be the subset of $\operatorname{Pol}_{1}\left(\left.\mathbf{A}\right|_{T}\right)$ consisting of those $R$-twins of the identity polynomial, $\mathrm{id}_{T}(x)$, which are permutations of $T$. If, for all $\langle\delta, \theta\rangle$-traces $T$, every $g \in H_{T, R}$ which satisfies $g(u) \delta u$ for some $u \in T$ also satisfies $g(x) \delta x$ on $T$, we will call $\langle\delta, \theta\rangle$ an $R$-regular tame quotient. Cyclic prime quotients are $R$-regular for all $R \subseteq A \times A$ since every $g(x) \in \operatorname{Pol}_{1}\left(\left.\mathbf{A}\right|_{T}\right)$ either satisfies $g(x) \delta x$ for all $x \in T$ or for no $x \in T$. Therefore cyclic prime quotients are 1-regular. We will find that the concepts of left and right nilpotence coincide for finite algebras whose type $\mathbf{1}$ prime quotients are 1-regular.

\section{Annihilating Tame Quotients}

Throughout this section we will be looking at the relationship between the four conditions (i) $C(\beta, \theta ; \delta),(i i)[\beta, \theta] \leq \delta,($ iii $) C(\theta, \beta ; \delta)$ and $(i v)[\theta, \beta] \leq \delta$. We will maintain the assumptions, without restating them each time, that $\beta, \delta$ and $\theta$ are congruences on the finite algebra $\mathbf{A},\langle\delta, \theta\rangle$ is a tame quotient of $\mathbf{A}$ and $U$ is a $\langle\delta, \theta\rangle$-minimal set with body $B$ and tail $T$.

Lemma 3.1 If $\operatorname{typ}(\delta, \theta) \in\{\mathbf{3}, \mathbf{4}, \mathbf{5}\}$, then for the conditions listed below $(i) \rightarrow(i i) \leftrightarrow($ iii $) \leftrightarrow$ (iv) holds.

(i) $C(\beta, \theta ; \delta)$. 
(ii) $[\beta, \theta] \leq \delta$.

(iii) $C(\theta, \beta ; \delta)$.

(iv) $[\theta, \beta] \leq \delta$.

If $\left.(\beta]^{k}\right|_{U} \subseteq B^{2} \cup T^{2}$ for some $k$, then all conditions are equivalent.

Proof: For the first step of the proof we will prove that whenever $\langle\delta, \theta\rangle$ is a nonabelian tame quotient we have

$$
C(\beta, \theta ; \delta) \leftrightarrow \beta \vee \delta \nsupseteq \theta
$$

and

$$
C(\theta, \beta ; \delta) \leftrightarrow \beta \wedge \theta \leq \delta
$$

We will use the fact that the tame quotients of $\mathbf{A}$ which have type $\mathbf{3}, \mathbf{4}$ or $\mathbf{5}$ are precisely the nonabelian prime quotients. This result is Theorem $5.7(2)$ of [4].

If $C(\beta, \theta ; \delta)$ holds, then since $C(\delta, \theta ; \delta)$ also holds we have $C(\beta \vee \delta, \theta ; \delta)$. But $C(\theta, \theta ; \delta)$ fails because $\langle\delta, \theta\rangle$ is nonabelian; therefore we must have $\beta \vee \delta \nsupseteq \theta$ or we contradict the monotonicity of the centralizer. On the other hand, $\beta \vee \delta \nsupseteq \theta$ implies that

$$
\theta \wedge(\beta \vee(\theta \wedge \delta))=\delta \leq \delta
$$

so by Theorem $2.1(v)$ we get that $C(\beta, \theta ; \delta)$ holds.

Now assume that $C(\theta, \beta ; \delta)$ holds. By the monotonicity of the centralizer we have $C(\beta \wedge$ $\theta, \beta \wedge \theta ; \delta)$. We also have $C(\beta \wedge \theta, \beta \wedge \theta ; \beta)$ clearly, so $C(\beta \wedge \theta, \beta \wedge \theta ; \beta \wedge \delta)$ holds. This means that $\beta \wedge \theta \stackrel{s}{\sim} \beta \wedge \delta$. Therefore,

$$
\delta=\delta \vee(\beta \wedge \delta) \stackrel{s}{\sim} \delta \vee(\beta \wedge \theta) \text { and } \delta \leq \delta \vee(\beta \wedge \theta) \leq \theta
$$

But $\delta \prec \theta$ and $\delta \stackrel{\&}{*} \theta$, so we must have $\delta=\delta \vee(\beta \wedge \theta)$ and therefore $\beta \wedge \theta \leq \delta$. Conversely, if $\beta \wedge \theta \leq \delta$ holds, then

$$
\beta \wedge(\theta \vee(\beta \wedge \delta)) \leq \delta
$$

so $C(\theta, \beta ; \delta)$.

We have already shown enough to conclude that $(i) \rightarrow($ iii $)$; i.e., $C(\beta, \theta ; \delta) \rightarrow C(\theta, \beta ; \delta)$; since

$$
\beta \vee \delta \nsupseteq \theta \rightarrow(\beta \vee \delta) \wedge \theta=\delta \rightarrow \beta \wedge \theta \leq \delta .
$$

In addition, the type of argument used in the last paragraph shows that $(i i),(i v) \rightarrow(i i i)$. Specifically, if $($ iii $)(=C(\theta, \beta ; \delta))$ fails, then $\beta \wedge \theta \not \leq \delta$ so, as argued above, $\beta \wedge \delta \mathcal{\psi} \beta \wedge \theta$. Hence $[\beta \wedge \theta, \beta \wedge \theta] \not \leq \delta$. Since $[\beta, \theta]$ and $[\theta, \beta]$ are $\geq[\beta \wedge \theta, \beta \wedge \theta]$ we must have $[\beta, \theta] \not \leq \delta$ and $[\theta, \beta] \not \leq \delta$.

As mentioned before this lemma, $(i) \rightarrow(i i)$ and $(i i i) \rightarrow(i v)$ follow from the definitions. At this point, therefore, we have proven that $(i) \rightarrow(i i) \rightarrow(i i i) \leftrightarrow(i v)$. To show that $($ iii $) \rightarrow($ ii $)$ assume that $\beta \wedge \theta \leq \delta$ (which is equivalent to $(i i i)$ ). Then $[\beta, \theta] \leq \beta \wedge \theta \leq \delta$ and we are done with the first claim of the lemma.

To finish, assume that $\left.(\beta]^{k}\right|_{U} \subseteq B^{2} \cup T^{2}$ for some $k$. Let us first simplify matters by showing that this condition implies the apparently stronger condition that $\left.\beta\right|_{U} \subseteq B^{2} \cup T^{2}$. 
Assume otherwise that $\left.\beta\right|_{U} \nsubseteq B^{2} \cup T^{2}$. If $\lambda$ is a minimal congruence below $\beta$ such that $\left.\lambda\right|_{U} \not \subset B^{2} \cup T^{2}$, then $\lambda \stackrel{\leq}{\not}(\beta]^{k}$. Since $\beta \stackrel{s}{\sim}(\beta]^{k}$, we get that $\lambda \stackrel{s}{\sim} \lambda \wedge(\beta]^{k}<\lambda$. Choose $\gamma$ such that $\lambda \wedge(\beta]^{k} \leq \gamma \prec \lambda$. By our choices, $\left.\gamma\right|_{U} \subseteq B^{2} \cup T^{2}$ and $\operatorname{typ}(\gamma, \lambda) \in\{\mathbf{1}, \mathbf{2}\}$. We can find $a \in T$ and $u \in B$ such that $S=\{a, u\}$ is contained in a $\langle\gamma, \lambda\rangle$-trace. $\left.\mathbf{A}\right|_{S}$ is abelian by Exercise 5.11 (1) of [4]. Thus, $\left.\mathbf{A}\right|_{S}$ has no binary semilattice polynomial. But, if $\operatorname{typ}(\delta, \theta) \in\{\mathbf{3}, \mathbf{4}\}$ or if $\operatorname{typ}(\delta, \theta)=\mathbf{5}$ and $u \in I$ where $I$ is the $\left.\delta\right|_{U^{-c l a s s}}$ of $U$ described by Lemma 4.15 (1) of [4], then $S$ is closed under a binary semilattice polynomial of $\mathbf{A}$. This is proved by Lemmas 4.15 (3) and 4.17 (3) of [4]. We must have that $\operatorname{typ}(\delta, \theta)=\mathbf{5}$ and that $u$ belongs to the $\left.\delta\right|_{U}$-class of $U$ which is labeled $O$ in Lemma 4.15 of [4]. The pseudo-meet polynomial, $p(x, y)$, of $\left.\mathbf{A}\right|_{U}$ satisfies $p(x, x)=x$ and $p(x, p(x, y))=p(x, y)$ on $U$ and also $p(a, u) \delta p(u, a) \delta a$. Let $a^{\prime}=p(u, a)$. Then $\left(u, a^{\prime}\right)=(p(u, u), p(u, a)) \in \lambda \cap(B \times T)$, so $\left(u, a^{\prime}\right) \in \lambda-\gamma$. Also,

$$
p\left(u, a^{\prime}\right)=a^{\prime}=p\left(a^{\prime}, a^{\prime}\right)
$$

and

$$
p(u, u)=u \neq p\left(a^{\prime}, u\right)\left(=a^{\prime \prime} \delta a^{\prime}\right) .
$$

Since $\left(u, a^{\prime \prime}\right) \in \lambda \cap(B \times T)$ we get that $\left(u, a^{\prime \prime}\right) \in \lambda-\gamma$ and, consequently, $[\lambda, \lambda] \not \leq \gamma$. This contradicts $\operatorname{typ}(\gamma, \lambda) \in\{\mathbf{1}, \mathbf{2}\}$. This contradiction succeeds in showing that $\left.(\beta]^{k}\right|_{U} \subseteq B^{2} \cup T^{2}$ for some $k$ is equivalent to $\left.\beta\right|_{U} \subseteq B^{2} \cup T^{2}$ when $\operatorname{typ}(\delta, \theta) \in\{\mathbf{3}, \mathbf{4}, \mathbf{5}\}$.

We may now assume that $\left.\beta\right|_{U} \subseteq B^{2} \cup T^{2}$ and that $\beta \wedge \theta \leq \delta$ to prove that $\beta \vee \delta \nsupseteq \theta$. It suffices to prove it on $U$, so assume instead that $\left.\left.\beta\right|_{U} \vee \delta\right|_{U} \geq\left.\theta\right|_{U}$. The body of $U$ is a single $\left.\theta\right|_{U}$-class and also a union of $\left.\beta\right|_{U^{-c}}$-lasses and (exactly 2 ) $\left.\delta\right|_{U^{-c l a s s e s}}$. It follows that

$$
B \times B=\left.\theta\right|_{B}=\left.\left.\left.\delta\right|_{B} \circ \beta\right|_{B} \circ \delta\right|_{B}
$$

If $\left.(b, c) \in \theta\right|_{B}-\left.\delta\right|_{B}$, then we can find $d, e \in B$ such that

$$
\left.\left.b \delta\right|_{B} d \beta\right|_{B} \text { e }\left.\delta\right|_{B} c
$$

But now $\left.(d, e) \in\left(\left.\theta\right|_{U}-\left.\delta\right|_{U}\right) \cap \beta\right|_{U}$. This contradicts our assumption that $\beta \wedge \theta \leq \delta$ and finishes the proof.

Lemma 3.2 If $\operatorname{typ}(\delta, \theta)=\mathbf{2}$, then for the conditions listed below $(i) \rightarrow(i i) \rightarrow(i i i) \leftrightarrow(i v)$ holds.

(i) $C(\beta, \theta ; \delta)$.

(ii) $[\beta, \theta] \leq \delta$.

(iii) $C(\theta, \beta ; \delta)$.

(iv) $[\theta, \beta] \leq \delta$.

If $\left.(\beta]^{k}\right|_{U} \subseteq B^{2} \cup T^{2}$ for some $k$, then all conditions are equivalent. 
Proof: Verification of our first statement only requires showing that either of the assumptions $[\beta, \theta] \leq \delta$ or $[\theta, \beta] \leq \delta$ implies $C(\theta, \beta ; \delta)$. Assume that $C(\theta, \beta ; \delta)$ fails. Then $C\left(N^{2}, \beta ; \delta\right)$ fails for $N$ equal to any $\langle\delta, \theta\rangle$-trace. There is a $\langle\delta, \theta\rangle$-trace $N \subseteq U$, a polynomial $p(x, \bar{y}) \in \operatorname{Pol}_{n+1}$ A and pairs $(u, v) \in N \times N,\left(a_{i}, b_{i}\right) \in \beta$ such that

$$
p(u, \bar{a}) \delta p(u, \bar{b})
$$

while

$$
p(v, \bar{a}) \theta-\delta p(v, \bar{b}) .
$$

We may assume that $p\left(A, A^{n}\right) \subseteq U$. All four elements in the last two displayed equivalences are $\left.\theta\right|_{U}$-related, but not all are $\left.\delta\right|_{U}$-related. Hence they all lie in some trace which we may assume is $N$. Let $d(x, y, z)$ be the pseudo-malcev operation of $U$ and define $p^{\prime}(x, \bar{y})=$ $d(p(x, \bar{y}), p(x, \bar{a}), p(v, \bar{a}))$. With this polynomial we have

$$
p^{\prime}(u, \bar{a})=p(v, \bar{a})=p^{\prime}(v, \bar{a})
$$

and

$$
p^{\prime}(u, \bar{b}) \delta p(v, \bar{a}) \theta-\delta p(v, \bar{b})=p^{\prime}(v, \bar{b}) .
$$

This shows that $[\beta, \theta] \not \leq \delta$ and establishes that $(i i) \rightarrow($ iii $)$. Continuing on, notice that since $N$ is closed under $d$ the elements $p^{\prime}(u, \bar{a}), p^{\prime}(v, \bar{a}), p^{\prime}(u, \bar{b})$ and $p^{\prime}(v, \bar{b})$ all belong to $N$. We can repeat the kind of argument we just used by letting $p^{\prime \prime}(x, \bar{y})=d\left(p^{\prime}(x, \bar{y}), p^{\prime}(u, \bar{y}), p^{\prime}(u, \bar{b})\right)$. Then

$$
p^{\prime \prime}(u, \bar{a})=p^{\prime}(u, \bar{b})=p^{\prime \prime}(u, \bar{b})
$$

and

$$
p^{\prime \prime}(v, \bar{a})=p^{\prime}(u, \bar{b}) \theta-\delta p^{\prime}(v, \bar{b})=p^{\prime \prime}(v, \bar{b}) .
$$

We use the fact that $p^{\prime}(u, \bar{b}) \theta-\delta p^{\prime}(v, \bar{b})$ which follows from $p^{\prime}(u, \bar{b}) \delta p^{\prime}(v, \bar{a}) \theta-\delta p^{\prime}(v, \bar{b})$. Now we've shown that $[\theta, \beta] \not \leq \delta$ too, so $(i v) \rightarrow($ iii $)$.

For the second statement of the lemma we will show that $(i v) \rightarrow(i)$ if $\left.(\beta]^{k}\right|_{U} \subseteq B^{2} \cup T^{2}$ for some $k$. As in the proof of Lemma 3.1, it can be shown that this hypothesis on $(\beta]^{k}$ is equivalent to the assumption that $\left.\beta\right|_{U} \subseteq B^{2} \cup T^{2}$. This can be proved by invoking Lemma 4.27 (4) (ii) of [4] which says, in the notation of the proof of Lemma 3.1, that since $\left.\gamma\right|_{U} \subseteq B^{2} \cup T^{2}$ and $\left.\lambda\right|_{U} \not \subset B^{2} \cup T^{2},\langle\gamma, \lambda\rangle$ is nonabelian. But if our claim that $\left.\beta\right|_{U} \subseteq B^{2} \cup T^{2}$ is false it can be shown (as in Lemma 3.1) that $\operatorname{typ}(\gamma, \lambda) \in\{\mathbf{1}, \mathbf{2}\}$. So now assume that $\left.\beta\right|_{U} \subseteq B^{2} \cup T^{2}$ and that $C(\beta, \theta ; \delta)$ fails. We can find a polynomial $p(x, \bar{y}) \in \operatorname{Pol}_{n+1} \mathbf{A}$ and pairs $(a, b) \in \beta$, $\left(u_{i}, v_{i}\right) \in \theta$ such that

$$
p(a, \bar{u}) \delta p(a, \bar{v})
$$

while

$$
p(b, \bar{u}) \theta-\delta p(b, \bar{v}) .
$$

We may assume that $p\left(A, A^{n}\right) \subseteq U$. The two elements in the last displayed equivalence belong to a trace of $U$ and, since the elements of the penultimate displayed equivalence are $\left.\beta\right|_{U}$-related to these elements, all four elements belong to the body of $U$. Now we can argue as in the early part of this proof: let $p^{\prime}(x, \bar{y})=d(p(x, \bar{y}), p(x, \bar{u}), p(b, \bar{u}))$. With this polynomial we have

$$
p^{\prime}(a, \bar{u})=p(b, \bar{u})=p^{\prime}(b, \bar{u})
$$


and

$$
p^{\prime}(a, \bar{v}) \delta p(b, \bar{u}) \theta-\delta p(b, \bar{v})=p^{\prime}(b, \bar{v}) .
$$

Thus, $[\theta, \beta] \not \subset \delta$ showing that $(i v) \rightarrow(i)$. From the first part of the lemma we conclude that if $\left.(\beta]^{k}\right|_{U} \subseteq B^{2} \cup T^{2}$ for some $k$, then all conditions are equivalent.

What is left is to analyze the situation when $\operatorname{typ}(\delta, \theta)=\mathbf{1}$. It turns out that in this situation there are no non-obvious relationships between the four properties that we are considering. (The obvious ones are $(i) \rightarrow(i i)$ and $(i i i) \rightarrow(i v)$. Showing that no others exist requires four counterexamples: one must show that $(i) \nrightarrow(i v),(i i) \nrightarrow(i),(i i i) \nrightarrow(i i)$ and $(i v) \nrightarrow($ iii $)$. Such counterexamples can be found among the algebras of similarity type $\langle 2,1\rangle$ which have no more than 13 elements.) However, there are two non-obvious implications under the hypothesis that $\left.(\beta]^{k}\right|_{U} \subseteq B^{2} \cup T^{2}$. This explains our wording of Lemmas 3.1 and 3.2. When $\operatorname{typ}(\delta, \theta) \neq \mathbf{1}$ it turns out that the hypotheses

1. $\left.\beta\right|_{U} \subseteq B^{2} \cup T^{2}$.

2. $\left.(\beta]^{k}\right|_{U} \subseteq B^{2} \cup T^{2}$ for some $k$.

3. $\left.[\beta]^{l}\right|_{U} \subseteq B^{2} \cup T^{2}$ for some $l$.

are equivalent. They are not equivalent when $\operatorname{typ}(\delta, \theta)=\mathbf{1}$ and for the results of this paper the second hypothesis seems to be the right one. This will become clear in the proof of Lemma 3.3. Moreover, if $\operatorname{typ}(\delta, \theta) \notin\{\mathbf{1}, \mathbf{5}\}$, then one can show that $C(\beta, \theta ; \delta)$ is equivalent to the conjunction of $C(\theta, \beta ; \delta)$ and $\left.(\beta]^{k}\right|_{U} \subseteq B^{2} \cup T^{2}$ for some $k$, so the hypothesis on $(\beta]^{k}$ is natural for the second claim in both Lemma 3.1 and Lemma 3.2.

Lemma 3.3 If $\operatorname{typ}(\delta, \theta)=1$ and $\left.(\beta]^{k}\right|_{U} \subseteq B^{2} \cup T^{2}$ for some $k$, then for the conditions listed below $($ iii $) \rightarrow($ iv $) \rightarrow(i) \leftrightarrow($ ii $)$ holds.

(i) $C(\beta, \theta ; \delta)$.

(ii) $[\beta, \theta] \leq \delta$.

(iii) $C(\theta, \beta ; \delta)$.

(iv) $[\theta, \beta] \leq \delta$.

Proof: We must prove that each of the conditions $[\theta, \beta] \leq \delta$ and $[\beta, \theta] \leq \delta$ imply that $C(\beta, \theta ; \delta)$ holds if $\left.(\beta]^{k}\right|_{U} \subseteq B^{2} \cup T^{2}$ for some $k$. Assume that $C(\beta, \theta ; \delta)$ fails. There is a polynomial $p(x, \bar{y}) \in \operatorname{Pol}_{n+1} \mathbf{A},(a, b) \in \beta$ and $\left(u_{i}, v_{i}\right) \in \theta$, for $i<n$, where

$$
p(a, \bar{u}) \delta p(a, \bar{v})
$$

but

$$
p(b, \bar{u}) \theta-\delta p(b, \bar{v}) .
$$

We may assume that $p\left(A, A^{n}\right) \subseteq U$. This implies that the elements $p(b, \bar{u})$ and $p(b, \bar{v})$ lie in a trace. 
Claim 1 The polynomial $p(x, \bar{y})$ which witnesses the fact that $C(\beta, \theta ; \delta)$ fails can be chosen to be binary.

Proof of Claim 1: We have $p\left(x, y_{0}, \ldots, y_{n-1}\right) \in \operatorname{Pol}_{n+1} \mathbf{A}$ such that $(p(a, \bar{u}), p(a, \bar{v})) \in \delta$ and $(p(b, \bar{u}), p(b, \bar{v})) \in \theta-\delta$. If $n=1$ we are done, so assume that $n>1$. Write $p_{a}(\bar{y})$ for the $n$-ary polynomial $p(a, \bar{y})$ and similarly write $p_{b}(\bar{y})$ for $p(b, \bar{y})$. Since $\operatorname{typ}(\delta, \theta)=$ $\mathbf{1}, \theta$ is strongly abelian over $\delta$. We also have $p_{a}\left(u_{0}, u_{1}, \ldots, u_{n-1}\right) \delta p_{a}\left(v_{0}, v_{1} \ldots, v_{n-1}\right)$, so from the strong term condition we get that $p_{a}\left(u_{0}, v_{1} \ldots, v_{n-1}\right) \delta p_{a}\left(v_{0}, v_{1}, \ldots, v_{n-1}\right)$. Further, $p_{b}\left(u_{0}, v_{1}, \ldots, v_{n-1}\right) \theta p_{b}\left(v_{0}, v_{1}, \ldots, v_{n-1}\right)$. If these last two elements are not $\delta$-congruent, then we can choose $p^{\prime}(x, y)=p\left(x, y, v_{1}, \ldots, v_{n}\right)$. Then

$$
p^{\prime}\left(a, u_{0}\right) \delta p^{\prime}\left(a, v_{0}\right),
$$

but

$$
p^{\prime}\left(b, u_{0}\right) \theta-\delta p^{\prime}\left(b, v_{0}\right) .
$$

This would finish the proof of the claim. Therefore, assume that

$$
p_{b}\left(u_{0}, v_{1}, \ldots, v_{n-1}\right) \delta p_{b}\left(v_{0}, v_{1}, \ldots, v_{n-1}\right) .
$$

In this case,

$$
p\left(a, u_{0}, u_{1}, \ldots, u_{n-1}\right) \delta p\left(a, v_{0}, v_{1}, \ldots, v_{n-1}\right) \delta p\left(a, u_{0}, v_{1}, \ldots, v_{n-1}\right)
$$

and

$$
p\left(b, u_{0}, u_{1}, \ldots, u_{n-1}\right) \theta-\delta p\left(b, v_{0}, v_{1}, \ldots, v_{n-1}\right) \delta p\left(b, u_{0}, v_{1}, \ldots, v_{n-1}\right) .
$$

Let $p^{\prime \prime}\left(x, y_{1}, \ldots, y_{n-1}\right)=p\left(x, u_{0}, y_{1}, \ldots, y_{n-1}\right)$. The previous two displayed equations prove that $p^{\prime \prime}$ is a polynomial which witnesses the fact that $C(\beta, \theta ; \delta)$ fails and $p^{\prime \prime}$ has arity smaller than the arity of $p$. Since this reduction can be accomplished whenever the arity of $p$ is more than 2 , we can assume that $p$ is a binary polynomial. This finishes Claim 1.

We now know that if $C(\beta, \theta ; \delta)$ fails, then we can find $p(x, y) \in \operatorname{Pol}_{2} \mathbf{A},(u, v) \in \theta-\delta$, $(a, b) \in \beta$ and a $\langle\delta, \theta\rangle$-minimal set $U$ such that $p(A, A) \subseteq U$ and $\left(p_{a}(u), p_{a}(v)\right) \in \delta$ while $\left(p_{b}(u), p_{b}(v)\right) \in \theta-\delta$. By Theorem 2.8 of [4], we know that $u$ and $v$ are connected modulo $\delta$ by $\langle\delta, \theta\rangle$-traces. Let $u=x_{0}, \ldots, x_{n}=v$ be a sequence of elements where, for each $i<n$, $\left(x_{i}, x_{i+1}\right) \in \delta$ or $\left\{x_{i}, x_{i+1}\right\}$ is a subset of some $\langle\delta, \theta\rangle$-trace. There is no loss of generality if we assume that this sequence is the shortest possible sequence among all sequences connecting elements $u^{\prime}$ and $v^{\prime}$ where $\left(u^{\prime}, v^{\prime}\right) \in \theta$ and for which there is a polynomial $p^{\prime}(x, y) \in \mathrm{Pol}_{2} \mathbf{A}$ and elements $\left(a^{\prime}, b^{\prime}\right) \in \beta$ such that $\left(p^{\prime}\left(a^{\prime}, u^{\prime}\right), p^{\prime}\left(a^{\prime}, v^{\prime}\right)\right) \in \delta$ and $\left(p^{\prime}\left(b^{\prime}, u^{\prime}\right), p^{\prime}\left(b^{\prime}, v^{\prime}\right)\right) \in \theta-\delta$. We shall now split our argument into two cases depending on whether this shortest sequence connecting $u$ and $v$ has only these two elements or whether all such sequences have more than two elements.

In Case 1 we have $u=x_{0}$ and $x_{1}=x_{n}=v$. We have $(u, v) \in N^{2}-\delta$ for some $\langle\delta, \theta\rangle$-trace $N$ which we may assume is a subset of $U$. Since $\left(p_{a}(u), p_{a}(v)\right) \in \delta$ and $(u, v) \in \theta-\delta$, it follows that $p_{a}(y)$ is not a permutation of $U$ and therefore $\left.p_{a}\left(\left.\theta\right|_{U}\right) \subseteq \delta\right|_{U}$. On the other hand, $\left(p_{b}(u), p_{b}(v)\right) \in \theta-\delta$ so $p_{b}(y)$ is a permutation of $U$. There is no harm with replacing $p_{x}(y)$ with an iterate $p_{x}\left(\cdots p_{x}(y) \cdots\right)=r_{x}(y)$ which satisfies $r_{x}\left(r_{x}(y)\right)=r_{x}(y)$ for all $x, y \in A$. 
All of our assumptions for $p_{x}(y)$ hold for $r_{x}(y)$ except that we also have that $r_{b}(y)$ is an idempotent permutation of $U$, so $r_{b}(y)=y$ on $U$.

To summarize, in Case 1 we have a $\langle\delta, \theta\rangle$-minimal set $U,(a, b) \in \beta, r(x, y) \in \operatorname{Pol}_{2} \mathbf{A}$ satisfying $r(A, A) \subseteq U$ and $r_{x}\left(r_{x}(y)\right)=r_{x}(y)$ for all $x, y \in A .\left.r_{a}\left(\left.\theta\right|_{U}\right) \subseteq \delta\right|_{U}$ and $r_{b}(y)=y$ on $U$. We must show that the situation described in this paragraph cannot happen if $[\theta, \beta] \leq \delta$ or $[\beta, \theta] \leq \delta$ holds (i.e., if condition $(i v)$ or (ii) holds).

If Case 1 does not occur, then for every choice of $p(x, y) \in \operatorname{Pol}_{2} \mathbf{A}$ and $(a, b) \in \beta$ we have $p_{a}\left(N^{2}\right) \subseteq \delta \leftrightarrow p_{b}\left(N^{2}\right) \subseteq \delta$ when $N$ is a $\langle\delta, \theta\rangle$-trace. For when this condition fails, we can choose any $(u, v) \in N^{2}-\delta$ and, after possibly interchanging $a$ and $b$, we are back in Case 1 . As mentioned earlier, in Case 2 the chain $x_{0}, \ldots, x_{n}$ connecting $u$ and $v$ has more than just the two elements $u$ and $v$. If $\left(p_{a}\left(x_{0}\right), p_{a}\left(x_{1}\right)\right) \in \delta$ and $\left(p_{b}\left(x_{0}\right), p_{b}\left(x_{1}\right)\right) \in \delta$, then after setting $u^{\prime}=x_{1}$ and $v^{\prime}=v$ we obtain a pair of elements connected by a shorter chain where

$$
p\left(a, u^{\prime}\right) \delta p(a, u) \delta p(a, v)=p\left(a, v^{\prime}\right)
$$

but

$$
p\left(b, u^{\prime}\right) \delta p(b, u) \theta-\delta p(b, v)=p\left(b, v^{\prime}\right) .
$$

This contradicts our minimality assumption about the sequence $x_{0}, \ldots, x_{n}$. We must have $\left(x_{0}, x_{1}\right) \in M^{2}$ for some $\langle\delta, \theta\rangle$-trace $M$ and either $p_{a}\left(M^{2}\right) \nsubseteq \delta$ or $p_{b}\left(M^{2}\right) \nsubseteq \delta$. In fact, both $p_{a}\left(M^{2}\right) \nsubseteq \delta$ and $p_{b}\left(M^{2}\right) \nsubseteq \delta$ must hold as we explained in the first sentence of this paragraph. A similar argument shows that $\left(x_{n-1}, x_{n}\right) \in M^{\prime 2}$ for some $\langle\delta, \theta\rangle$-trace $M^{\prime}$ and $p_{a}\left(M^{\prime 2}\right) \nsubseteq \delta$ and $p_{b}\left(M^{\prime 2}\right) \nsubseteq \delta$. Without loss of generality, we may assume that $M \subseteq U$. We cannot have $M^{\prime} \subseteq U$ also, since $u \in M \subseteq U, v \in M^{\prime},(u, v) \in \theta$ but $\left.(u, v) \notin \theta\right|_{U}$ or else we would be in Case 1. However, since $p_{a}\left(M^{2}\right) \nsubseteq \delta$ and $p_{a}\left(M^{\prime 2}\right) \nsubseteq \delta$ we get $M \simeq p_{a}(M)$ and $M^{\prime} \simeq p_{a}\left(M^{\prime}\right)$. Further, since $p_{a}(A) \subseteq U$ and $p_{a}(u) \delta p_{a}(v)$ we must have $p_{a}(M)=p_{a}\left(M^{\prime}\right)$. Similarly, since $p_{b}\left(M^{2}\right) \nsubseteq \delta, p_{b}\left(M^{\prime 2}\right) \nsubseteq \delta$ and $p_{b}(u) \theta p_{b}(v)$ we have $p_{b}(M)=p_{b}\left(M^{\prime}\right)$. Before summarizing all that we have described about Case 2, notice that $p_{x}(y)$ can be replaced by an iterate $r_{x}(y)$ satisfying $r_{x}\left(r_{x}(y)\right)=r_{x}(y)$ for all $x, y \in A$ without affecting any of our conclusions so far. On $U$ we have $r_{a}(y)=r_{b}(y)=y$ since $p_{a}(y)$ and $p_{b}(y)$ are permutations of $U$, so $r_{a}\left(M^{\prime}\right)=M=r_{b}\left(M^{\prime}\right)$. Further, $\left(r_{a}(u), r_{a}(v)\right) \in \delta,\left(r_{b}(u), r_{b}(v)\right) \in \theta-\delta$ and $r_{a}(u)=u=$ $r_{b}(u)$, implying that we have $\left(r_{a}(v), r_{b}(v)\right) \in \theta-\delta$.

To summarize Case 2 , if $C(\beta, \theta ; \delta)$ fails and we are not in Case 1 , then we can find $\langle\delta, \theta\rangle$ traces $M$ and $M^{\prime}$ contained in the same $\theta$-class with $v \in M^{\prime}, r(x, y) \in \operatorname{Pol}_{2} \mathbf{A}$ and $(a, b) \in \beta$ such that $r_{x}\left(r_{x}(y)\right)=r_{x}(y)$ and $\left(r_{a}(v), r_{b}(v)\right) \in \theta-\delta$ while $r_{a}(y)$ and $r_{b}(y)$ are polynomial isomorphisms of $M^{\prime}$ onto $M$. We need to show this too is impossible if $[\theta, \beta] \leq \delta$ or $[\beta, \theta] \leq \delta$.

The next two claims will show that each of the cases that we've reduced to leads to a contradiction.

Claim 2 If $U$ is a $\langle\delta, \theta\rangle$-minimal set, $(a, b) \in \beta, r(x, y) \in \operatorname{Pol}_{2} \mathbf{A}$ satisfies $r(A, A) \subseteq U$, $r_{x}\left(r_{x}(y)\right)=r_{x}(y)$ for all $x, y \in A$ and $\left.r_{a}\left(\left.\theta\right|_{U}\right) \subseteq \delta\right|_{U}$ while $r_{b}(y)=y$ on $U$; then $[\theta, \beta] \not \leq \delta$ and $[\beta, \theta] \not \leq \delta$.

Proof of Claim 2: For all $y \in U$ we have

$$
r_{a}\left(r_{a}(y)\right)=r_{a}(y) \beta r_{b}(y)=y .
$$


In particular, $\left(y, r_{a}(y)\right) \in \beta=(\beta]^{1}$. However, by changing the first occurrence of $a$ to $b$ in $r(a, y)=r(a, r(a, y))$ it follows that

$$
y=r(b, y)[\beta, \beta] r(b, r(a, y))=r(a, y),
$$

so in fact we have $(y, r(a, y)) \in(\beta]^{2}$ for all $y \in U$. We can iterate this argument as many times as we like to prove that $(y, r(a, y)) \in(\beta]^{k}$ for all $y \in U$ and all values of $k$. Choosing a $k$ so that $\left.(\beta]^{k}\right|_{U} \subseteq B^{2} \cup T^{2}$ we see that both the body and tail of $U$ are closed under $r_{a}(y)$. Choose $u \in r_{a}(B) \subset B$. If $N$ is the trace of $U$ which contains $u$, then since $\left.r_{a}\left(\left.\theta\right|_{U}\right) \subseteq \delta\right|_{U}$ and $r_{a}(u)=u$ we get $r_{a}(N) \subseteq u /\left.\delta\right|_{N}$. Pick $v \in N$ such that $(u, v) \in \theta-\delta$. Necessarily, $v \notin r_{a}(B)$, so set $w=r_{a}(v) \delta r_{a}(u)=u$. Since $v \theta-\delta u \delta w$ and we have

$$
r(a, v)=w=r(a, w)
$$

while

$$
r(b, v)=v \theta-\delta w=r(b, w),
$$

we deduce that $[\beta, \theta] \not \leq \delta$. On the other hand,

$$
r(a, w)=w=r(b, w)
$$

while

$$
r(a, v)=w \theta-\delta v=r(b, v)
$$

so $[\theta, \beta] \not \leq \delta$. This proves Claim 2. In fact it proves the stronger fact that under the hypotheses of Claim 2 there is a pair $(v, w) \in \theta-\delta$ such that $\left[\beta, \operatorname{Cg}^{\mathrm{A}}(v, w)\right]=\operatorname{Cg}^{\mathrm{A}}(v, w)=$ $\left[\mathrm{Cg}^{\mathrm{A}}(v, w), \beta\right]$.

We may assume that we are in Case 2. We take care of this case with the following claim.

Claim 3 Let $M$ and $M^{\prime}$ be $\langle\delta, \theta\rangle$-traces contained in the same $\theta$-class with $v \in M^{\prime}$. Suppose that $r(x, y) \in \mathrm{Pol}_{2} \mathbf{A},(a, b) \in \beta, r_{x}\left(r_{x}(y)\right)=r_{x}(y)$ and $\left(r_{a}(v), r_{b}(v)\right) \in \theta-\delta$ while $r_{a}(y)$ and $r_{b}(y)$ are polynomial isomorphisms of $M^{\prime}$ onto $M$. Then both $[\theta, \beta] \not \leq \delta$ and $[\beta, \theta] \not \leq \delta$.

Proof of Claim 3: Let $u=r_{a}(v) \in M$. Then

$$
r(a, u)=u=r(b, u)
$$

while

$$
r(a, v)=u \theta-\delta r(b, v),
$$

so $[\theta, \beta] \not \leq \delta$. Further,

$$
r(a, u)=u=r(a, v)
$$

while

$$
r(b, u)=u \theta-\delta r(b, v),
$$

so $[\beta, \theta] \not \leq \delta$. This proves Claim 3 .

To briefly summarize, we have shown that if $\left.(\beta]^{k}\right|_{U} \subseteq B^{2} \cup T^{2}$ holds for some $k$ and $C(\beta, \theta ; \delta)$ fails, then we can reduce to one of two special cases. Claims 2 and 3 show that if $[\theta, \beta] \leq \delta$ or $[\beta, \theta] \leq \delta$, then these cases cannot occur. Therefore, the theorem is proved.

We combine the results of Lemmas 3.1, 3.2 and 3.3 into a purely order-theoretic statement about the commutator and the centralizer relation. 
Theorem 3.4 If $\mathbf{A}$ is a finite algebra, $I[\delta, \theta]$ is a tight interval in $\mathbf{C o n} \mathbf{A}$ and $(\beta]^{k} \leq \theta$ for some $k$ and some $\beta \in$ Con $\mathbf{A}$, then

$$
[\theta, \beta] \leq \delta \rightarrow[\beta, \theta] \leq \delta \leftrightarrow C(\beta, \theta ; \delta)
$$

Proof: This follows immediately from Lemmas 3.1, 3.2 and 3.3. The hypothesis that $I[\delta, \theta]$ is a tight interval in Con $\mathbf{A}$ guarantees that $\langle\delta, \theta\rangle$ is tame (by Theorem 2.11 of [4]). Since the body and tail of any $\langle\delta, \theta\rangle$-minimal set are each unions of $\left.\theta\right|_{U}$-classes, $\left.\theta\right|_{U} \subseteq B^{2} \cup T^{2}$. Hence the hypothesis that $(\beta]^{k} \leq \theta$ for some $k$ is strong enough to guarantee that $\left.(\beta]^{k}\right|_{U} \subseteq$ $B^{2} \cup T^{2}$ for some $k$.

Armed with Lemmas 3.2 and 3.3 we can easily prove that right nilpotent congruences on a finite algebra are left nilpotent.

Theorem 3.5 Let $\mathbf{A}$ be a finite algebra. The right nilpotent congruences on $\mathbf{A}$ are also left nilpotent.

Proof: Assume otherwise that $\mathbf{A}$ has a congruence $\beta$ such that $[\beta)^{n}=0$, while $(\beta]^{k}=0$ for no value of $k$. The sequence

$$
(\beta]^{1} \geq(\beta]^{2} \geq \cdots \geq(\beta]^{k} \cdots
$$

is eventually constant and greater than 0 . Let $\theta=(\beta]^{l}$ be this constant value. Then $0<\theta \leq \beta,[\theta, \beta]<\theta$ but $[\beta, \theta]=\theta$.

Choose $\delta$ so that $[\theta, \beta] \leq \delta \prec \theta ;\langle\delta, \theta\rangle$ is tame. Since $[\theta, \theta] \leq[\theta, \beta] \leq \delta$ we must have $\operatorname{typ}(\delta, \theta) \in\{\mathbf{1}, \mathbf{2}\}$. If $U$ is a $\langle\delta, \theta\rangle$-minimal set, then we have $\left.(\beta]^{l}\right|_{U}=\left.\theta\right|_{U} \subseteq B^{2} \cup T^{2}$. Using either Lemma 3.2 or Lemma 3.3, depending on the type of $\langle\delta, \theta\rangle$, we find that $[\theta, \beta] \leq \delta$ implies that $[\beta, \theta] \leq \delta$ which certainly contradicts our earlier conclusion that $[\theta, \beta] \leq \delta$ while $[\beta, \theta]=\theta>\delta$. The assumption that $(\beta]^{l}=(\beta]^{l+1}>0$ must be false.

In the following corollary we will say that a congruence on a locally finite algebra is locally right (left) nilpotent if its restriction to any finite subalgebra is right (left) nilpotent.

Corollary 3.6 Every locally right nilpotent congruence on a locally finite algebra is locally left nilpotent.

An unusual aspect of the proof of Theorem 3.5 is that it says nothing about the the relationship between the right nilpotency class and the left nilpotency class of a right nilpotent congruence. We suspect that if a congruence on a finite algebra is $k$-step right nilpotent, then it is also $k$-step left nilpotent.

Problem 1 Show that if $\beta$ is a right nilpotent congruence on a finite algebra and $\alpha \leq \beta$, then $[\beta, \alpha] \leq[\alpha, \beta]$.

If the statement of Problem 1 is true, then it implies that the left nilpotency class of a right nilpotent congruence is less than or equal to its right nilpotency class. But how much less can it be? 
Problem 2 Find all pairs of integers $(l, r)$ such that there exists a finite algebra with a congruence which is $r$-step right nilpotent, $l$-step left nilpotent but not $(r-1)$-step right nilpotent nor $(l-1)$-step left nilpotent.

Let's say that a congruence on a finite algebra is $\infty$-step right (left) nilpotent if it is not right (left) nilpotent. Then any congruence on a finite algebra can be assigned a pair $(l, r)$ as in Problem 2 where $r, l \in \omega \cup\{\infty\}$. Let $\mathcal{N}$ be the set of all pairs that arise. Here are 5 things that we know about the pairs in $\mathcal{N}$.

(i) If $(l, r) \in \mathcal{N}$, then $l=1$ if and only if $r=1$. (Saying that either $l=1$ or $r=1$ is equivalent to saying that the congruence assigned $(l, r)$ is abelian.)

(ii) $(\infty, k) \notin \mathcal{N}$ for any $k \in \omega$. (By Theorem 3.5.)

(iii) $(k, k) \in \mathcal{N}$ for all $k \in \omega \cup\{\infty\}$. (There exist finite groups of any prescribed nilpotency class and groups have symmetric commutator.)

(iv) If we order $\omega \cup\{\infty\}$ by extending the usual order on $\omega$ to include $k<\infty$ for all $k \in \omega$, then $\mathcal{N}$ is a join subsemilattice of $(\omega \cup\{\infty\}) \times(\omega \cup\{\infty\})$. (To see this, assume that $\mathbf{A}$ is a finite algebra with a congruence $\beta$ which is assigned the pair $(l, r)$. Let $\mathbf{A}^{\prime}$ be a finite algebra with a congruence $\beta^{\prime}$ which is assigned $\left(l^{\prime}, r^{\prime}\right)$. Let $\mathbf{A}^{\prime \prime}=\mathbf{A} \times \mathbf{A}^{\prime} \in \mathcal{V}(\mathbf{A}) \otimes \mathcal{V}\left(\mathbf{A}^{\prime}\right)$ and let $\beta^{\prime \prime}=\beta \times \beta^{\prime}$. Then $\beta^{\prime \prime}$ is assigned $\left(l^{\prime \prime}, r^{\prime \prime}\right)=\left(\max \left(l, l^{\prime}\right), \max \left(r, r^{\prime}\right)\right)$.)

$(v)(k, \infty) \in \mathcal{N}$ for all $k \geq 2$. (By (iii) and (iv) it suffices to show that $(2, \infty) \in \mathcal{N}$. This is done in the following example.)

Example 1 This is an example of a 4-element algebra $\mathbf{A}$ on the set $A=\{u, v, w, a\}$ with a unary operation, $t(x)$, and a binary operation, $p(x, y)$. The unary operation is given by $t(u)$ $=v, t(v)=w, t(w)=u$ and $t(a)=a$. The $p$-table is:

\begin{tabular}{|c||c|c|c|c|}
\hline $\mathrm{p}$ & $\mathrm{u}$ & $\mathrm{v}$ & $\mathrm{w}$ & $\mathrm{a}$ \\
\hline \hline $\mathrm{u}$ & $\mathrm{u}$ & $\mathrm{v}$ & $\mathrm{w}$ & $\mathrm{a}$ \\
\hline $\mathrm{v}$ & $\mathrm{u}$ & $\mathrm{V}$ & $\mathrm{w}$ & $\mathrm{a}$ \\
\hline $\mathrm{w}$ & $\mathrm{u}$ & $\mathrm{V}$ & $\mathrm{w}$ & $\mathrm{a}$ \\
\hline $\mathrm{a}$ & $\mathrm{u}$ & $\mathrm{w}$ & $\mathrm{v}$ & $\mathrm{a}$ \\
\hline
\end{tabular}

A has only one proper non-trivial congruence, $\alpha$, which is determined by the partition $\{\{u, v, w\},\{a\}\}$ (henceforth we use the abbreviated notation: uvw/a). $\mathbf{A} / \alpha$ is term equivalent to the 2-element set and so is abelian. Therefore $[1,1] \leq \alpha$ in Con A. However,

$$
p(u, u)=p(a, u) \quad \text { and } \quad p(u, w) \neq p(a, w),
$$

so $[\alpha, 1]>0$. It follows that $\alpha=[\alpha, 1]=[1,1]=[1)^{k}$ for all $k$. On the other hand, we claim that $0=[1, \alpha]=(1]^{3}$. To prove this, let $\mathbf{B}$ be the subalgebra of $\mathbf{A}^{2}$ whose universe is $\alpha$. Proving that $C(1, \alpha ; 0)$ holds in $\mathbf{A}$ is equivalent to proving that the diagonal of $\mathbf{A}^{2}, \Delta=$ $\{(u, u),(v, v),(w, w),(a, a)\}$, is a block of a congruence on $\mathbf{B}$.

Let $\beta$ be the equivalence relation on $B$ defined by $\langle(r, s),(x, y)\rangle \in \beta$ if and only if

$$
(r, s) \in \Delta \longleftrightarrow(x, y) \in \Delta
$$


holds. The diagonal and its complement in $B$ are the only $\beta$-blocks, so we will be done if we can prove that $\beta$ is a congruence. Since $t$ is a unary permutation of $A$, it is also a permutation of $B$ which leaves $\Delta$ invariant. It must leave $B-\Delta$ invariant also, so $\beta$ is compatible with $t$. Now we ask the reader to verify that for all $(r, s),(x, y) \in B(=\alpha)$ we have

$$
p(r, x)=p(s, y) \longleftrightarrow x=y .
$$

This is equivalent to saying that in $\mathbf{B}$ we have

$$
p((r, s),(x, y)) \in \Delta \longleftrightarrow(x, y) \in \Delta .
$$

The compatibility of $\beta$ with $p$ follows immediately from this. This finishes that proof that $(1]^{3}=0$ even though $[1)^{k}=\alpha>0$ for all $k>1$.

We will find in the next section that to construct left nilpotent algebras which are not right nilpotent it is necessary to introduce non-cyclic subtypes. Therefore, it is worth pointing out that the algebra in Example 1 can be easily modified so that it is still left nilpotent, not right nilpotent, $\langle 0, \alpha\rangle$ is the only non-cyclic prime quotient and $\operatorname{typ}(0, \alpha)=\mathbf{1}_{(\mathbf{G}, \mathbf{H})}$ where the only restriction on $(\mathbf{G}, \mathbf{H})$ is that it be a non-cyclic subtype of a prime quotient. Here is how to do it.

Example 2 Let $N$ be any non-cyclic simple G-set over the group $G$. Let $H$ be the subgroup of $G$ which fixes some $u \in N$. Since $N$ is a non-cyclic G-set, $H$ contains an element, $h$, which is not the identity permutation of $U$. Let $g_{0}, \ldots, g_{n-1} \in G$ be a set of elements such that $\left\{h, g_{0}, \ldots, g_{n-1}\right\}$ generates $G$. Let $a$ be any symbol not in $N \cup G$. We will define an algebra A with one binary operation, $p(x, y)$, and $n$ unary operations on the set $A=N \cup\{a\}$.

For each $g_{i}$ chosen above, define a unary operation $t_{i}(x)$ on $A$ by $t_{i}(a)=a$ and, for $x \in N$, $t_{i}(x)=g_{i}(x)$. The $p$ operation can be described as follows. If $x \neq a$ or $x=a=y$, then $p(x, y)=y$. If $x=a$ and $y \neq a$, then $p(x, y)=h(y)$.

The algebra in Example 1 was constructed in exactly the way just described. The same arguments used there apply here and show that:

(i) There is exactly one non-trivial congruence on $\mathbf{A}$ and it is determined by the partition $N / a$.

(ii) $[1,1]=\alpha=[\alpha, 1]=[1)^{k}$ for all $k$.

(iii) $(1]^{3}=0$.

Further, it is easy to verify that $A$ is a $\langle 0, \alpha\rangle$-minimal set whose only trace is $N$. The non-constant unary polynomials of $\left.\mathbf{A}\right|_{N}$ are generated by $\left\{p(a, x), t_{0}(x), \ldots, t_{n-1}(x)\right\}$ and from this it follows that $\left.\mathbf{A}\right|_{N}$ is polynomially equivalent to the G-set $\langle N ; G\rangle$.

Examples 1 and 2 prove that left nilpotence need not imply right nilpotence for finite algebras. For infinite algebras left and right nilpotence are independent of each other. To show that left nilpotence need not imply right nilpotence for infinite algebras, let $\mathbf{S}$ be an infinite algebra of similarity type $\langle 2,1\rangle$ which is term equivalent to a set. If $\mathbf{A}$ is the algebra of Example 1, then $\mathbf{A} \times \mathbf{S}$ is 2-step left nilpotent and not right nilpotent. To prove that right nilpotence need not imply left nilpotence for infinite algebras we give the following counterexample. 
Example 3 Our counterexample is countably infinite and has similarity type $\langle 2,1,1,1\rangle$. The algebra, which we denote by $\mathbf{A}$, will have the set $A=Z \cup\{\infty\}=\{\cdots,-2,-1,0,1,2, \cdots\} \cup$ $\{\infty\}$ for its universe. $Z$ is also the universe for the ring of integers and we will freely refer to the ring operations of $\mathbf{Z}$ in the construction of this example. The unary operations of $\mathbf{A}$ are $r(x), s(x)$ and $t(x)$. We set $r(\infty)=s(\infty)=t(\infty)=\infty$ while for $x \neq \infty$ we set $r(x)=$ $x+1, s(x)=x-1$ and

$$
t(x)= \begin{cases}x & \text { if } x \leq 0 \\ x+1 & \text { if } x>0 \text { and } x \text { is odd } \\ x-1 & \text { if } x>0 \text { and } x \text { is even. }\end{cases}
$$

The binary operation symbol is $p$ and we define

$$
p(x, y)= \begin{cases}3 y & \text { if } y \neq \infty \\ 3 x+1 & \text { if } x \neq \infty=y \\ 2 & \text { if } x=y=\infty\end{cases}
$$

We now argue that $[[1,1], 1]=0$, but $(1]^{k}=[1,1]>0$ for all $k$.

The operations $r, s$ and $t$ are permutations of $A$. If $G$ is the group of permutations of of $A$ generated by these operations, then $A$ is the union of exactly two $G$-orbits: $Z$ and $\{\infty\}$. We claim that $G$ acts primitively and transitively on $Z$. Clearly the subgroup of $G$ generated by $r$ and $s$ already acts transitively on $Z$. To show that $G$ acts primitively, let $R$ be a non-trivial equivalence relation on $Z$ which is preserved by $G$. Select $(x, y) \in R$ so that $x-y>0$ and this difference is as small as possible. Using $r$ and $s$ we can arrange it so that $y=0$, so assume that $(x, y)=(x, 0)$ was our original pair. Now $(t(x), t(y))=(t(x), 0) \in R$ and therefore $(x, t(x)),(t(x), x) \in R$. Since $|x-t(x)|=1$, we must have had $x=1$ above. Thus, $(x, y)=(1,0) \in R$. Using $r$ and $s$ we get $(n, n+1) \in R$ for all $n \in Z$, so $R=Z \times Z$; i.e., $G$ acts primitively on $Z$.

Assume that $\alpha$ is a congruence on $\mathbf{A}$. If $(n, \infty) \in \alpha$, then $(r(n), r(\infty))=(n+1, \infty) \in \alpha$ and so $(n, n+1) \in \alpha . \mathrm{Pol}_{1} \mathbf{A}$ contains $G$, so $Z \times Z \subseteq \alpha$. Hence $\alpha=1$ if the congruence class $\infty / \alpha$ is not equal to $\{\infty\}$. In other words, if $\alpha$ is a proper congruence, then $\alpha=$ $\left.\alpha\right|_{Z} \cup\{(\infty, \infty)\}$. But $\left.\alpha\right|_{Z}=0_{Z}$ or $1_{Z}$ since $G \subseteq \mathrm{Pol}_{1} \mathbf{A}$. Therefore, the congruences of $\mathbf{A}$ are just $0_{\mathbf{A}}, \alpha=Z \times Z \cup\{(\infty, \infty)\}$ and $1_{\mathbf{A}}$. We will prove that $[1,1]=\alpha,[\alpha, 1]=0$ and $[1, \alpha]=\alpha$ to show that $\mathbf{A}$ is right nilpotent, but not left nilpotent.

First,

$$
p(0,0)=0=p(1,0)
$$

but

$$
p(0, \infty)=1 \neq 4=p(1, \infty)
$$

so, since $(0, \infty) \in 1$ and $(0,1) \in \alpha, C(1, \alpha ; 0)$ fails. It follows that $[1,1] \geq[1, \alpha]>0$. On the other hand, $\mathbf{A} / \alpha$ is term equivalent to a 2-element pointed set which is abelian. This implies that $C(1,1 ; \alpha)$ holds, whence $\alpha \geq[1,1] \geq[1, \alpha] \geq \alpha$. We deduce that $[1,1]=[1, \alpha]=\alpha$. We now need to show that $[\alpha, 1]=0$ to finish. This requires showing that there is a congruence on $\mathbf{A}^{2}$ which has $\Delta_{Z}=\left\{(z, z) \in A^{2} \mid z \in Z\right\}$ and $\Delta_{\infty}=\{(\infty, \infty)\}$ as congruence blocks. Let $\Delta=\Delta_{Z} \cup \Delta_{\infty}$ and let $\beta$ be the equivalence relation on $A^{2}$ defined by $\langle(r, s),(x, y)\rangle \in \beta$ if and only if $(r, x),(s, y) \in \alpha$ and

$$
(r, s) \in \Delta \longleftrightarrow(x, y) \in \Delta .
$$


The blocks of $\beta$ are $\Delta_{\infty}, \Delta_{Z}, Z \times Z-\Delta_{Z},\{\infty\} \times Z$, and $Z \times\{\infty\}$. To see that $\beta$ is a congruence on $\mathbf{A}^{2}$ we must show that it is invariant under $r, s, t$ and $p$. Arguing as we did in Example 1, the fact that $r, s$ and $t$ are permutations of $A$ which fix $\infty$ is enough to prove that $\beta$ is invariant under these operations. Now, looking back to the way $p$ is defined, one can see that for all $(u, v),(x, y) \in A^{2}$ we have $p((u, v),(x, y)) \in Z \times Z$ so

$$
p((u, v),(x, y)) \in \Delta_{Z} \leftrightarrow p((u, v),(x, y)) \notin Z \times Z-\Delta_{Z} .
$$

These equivalent conditions are also equivalent to saying that either

(i) $(x, y) \in \Delta_{Z}$ or else

(ii) $(x, y) \in \Delta_{\infty}$ and $(u, v) \in \Delta$.

Now suppose that $\left\langle\left(u_{1}, v_{1}\right),\left(u_{2}, v_{2}\right)\right\rangle,\left\langle\left(x_{1}, y_{1}\right),\left(x_{2}, y_{2}\right)\right\rangle \in \beta$. If $p\left(\left(u_{1}, v_{1}\right),\left(x_{1}, y_{1}\right)\right) \in \Delta_{Z}$ and $(i)\left(x_{1}, y_{1}\right) \in \Delta_{Z}$, then $\left(x_{2}, y_{2}\right) \in \Delta_{Z}$ so $p\left(\left(u_{2}, v_{2}\right),\left(x_{2}, y_{2}\right)\right) \in \Delta_{Z}$. On the other hand, if $p\left(\left(u_{1}, v_{1}\right),\left(x_{1}, y_{1}\right)\right) \in \Delta_{Z}$ and $\left(x_{1}, y_{1}\right) \notin \Delta_{Z}$, we must have $(i i)\left(x_{1}, y_{1}\right) \in \Delta_{\infty}$ and $\left(u_{1}, v_{1}\right) \in \Delta$. In this case, $\left(x_{2}, y_{2}\right) \in \Delta_{\infty}$ and $\left(u_{2}, v_{2}\right) \in \Delta$. Hence, $p\left(\left(u_{2}, v_{2}\right),\left(x_{2}, y_{2}\right)\right) \in \Delta_{Z}$ holds. In either case, if $\left\langle\left(u_{1}, v_{1}\right),\left(u_{2}, v_{2}\right)\right\rangle,\left\langle\left(x_{1}, y_{1}\right),\left(x_{2}, y_{2}\right)\right\rangle \in \beta$ we have

$$
p\left(\left(u_{1}, v_{1}\right),\left(x_{1}, y_{1}\right)\right) \in \Delta_{Z} \longrightarrow p\left(\left(u_{2}, v_{2}\right),\left(x_{2}, y_{2}\right)\right) \in \Delta_{Z}
$$

By symmetry the reverse implication holds, too. So in fact

$$
p\left(\left(u_{1}, v_{1}\right),\left(x_{1}, y_{1}\right)\right) \in \Delta_{Z} \longleftrightarrow p\left(\left(u_{2}, v_{2}\right),\left(x_{2}, y_{2}\right)\right) \in \Delta_{Z} .
$$

Since $p\left(A^{2}, A^{2}\right) \subseteq Z^{2}$, we also get

$$
p\left(\left(u_{1}, v_{1}\right),\left(x_{1}, y_{1}\right)\right) \in Z^{2}-\Delta_{Z} \longleftrightarrow p\left(\left(u_{2}, v_{2}\right),\left(x_{2}, y_{2}\right)\right) \in Z^{2}-\Delta_{Z} .
$$

The last two bi-implications prove that if $\left\langle\left(u_{1}, v_{1}\right),\left(u_{2}, v_{2}\right)\right\rangle,\left\langle\left(x_{1}, y_{1}\right),\left(x_{2}, y_{2}\right)\right\rangle \in \beta$, then $\left\langle p\left(\left(u_{1}, v_{1}\right),\left(x_{1}, y_{1}\right)\right), p\left(\left(u_{2}, v_{2}\right),\left(x_{2}, y_{2}\right)\right)\right\rangle \in \beta$. We conclude that $\beta$ is a congruence and therefore $[\alpha, 1]=0$.

We end this section with a further application of Lemmas 3.2 and 3.3. In 1983, David Hobby proved that any finite algebra whose congruence lattice is isomorphic to the lattice $\mathbf{M}_{\mathbf{n}}, n \geq 3$, is abelian. ( $\mathbf{M}_{\mathbf{n}}$ is the lattice of height 2 with $n$ atoms.) This result, which is false for infinite algebras, was an early birth pang for the theory developed in [4]: "The Structure of Finite Algebras: Tame Congruence Theory" by Hobby and Ralph McKenzie. The proud parents of this new theory ended their volume with a list of hard questions, most of which are still unsolved. The first to be settled was a variation on Hobby's discovery. It was Problem 4 of [4] which asked essentially: If $\mathbf{A}$ is a finite algebra and $\mathbf{M}_{\mathbf{n}}, n \geq 3$, is isomorphic to a 0,1-sublattice of Con $\mathbf{A}$, then must $\mathbf{A}$ be abelian? It is a consequence of tame congruence theory that any finite algebra $\mathbf{A}$ whose congruence lattice has $\mathbf{M}_{\mathbf{n}}, n \geq 3$, as a 0,1 -sublattice must be at least solvable. Further, it can be shown that if $\mathbf{1} \notin \operatorname{typ}\{\mathbf{A}\}, \mathbf{A}$ must even be abelian. But with [7], Ross Willard showed how to construct finite nonabelian algebras whose congruence lattice had $\mathbf{M}_{\mathbf{n}}$ as a 0,1 -sublattice. In this section we complement 
Willard's negative result with a positive one: If the congruence lattice of a finite algebra $\mathbf{A}$ has $\mathbf{M}_{\mathbf{n}}, n \geq 3$, as a 0,1 -sublattice, then $\mathbf{H}(\mathbf{A})$ is left nilpotent.

Our arguments work for some lattices other than just $\mathbf{M}_{\mathbf{n}}$. Let $\mathcal{K}$ denote the class of all non-trivial finite lattices which satisfy

$$
\bigvee\{\beta \mid \bigvee\{\gamma \mid \gamma \wedge \beta=0\}=1\}=1
$$

$\mathcal{K}$ contains each $\mathbf{M}_{\mathbf{n}}$ for $n \geq 3$. Further, if a finite lattice $\mathbf{L}$ contains a member of $\mathcal{K}$ as a 0,1 -sublattice, then $\mathbf{L}$ itself belongs to $\mathcal{K}$. That is, $\mathcal{K}$ is closed under the formation of finite "0,1-extensions". However, $\mathcal{K}$ is more than just the class of finite 0,1 -extensions of $\mathbf{M}_{\mathbf{3}}$. For example, $\mathcal{K}$ contains every finite lattice which has a spanning $n$-frame, $n>1$, and therefore all subspace lattices of finite projective geometries are in $\mathcal{K}$. For even dimensional geometries these lattices have no 0,1-sublattices isomorphic to any $\mathbf{M}_{\mathbf{n}}$. We will prove that if $\mathbf{A}$ is finite and Con $\mathbf{A} \in \mathcal{K}$, then $\mathbf{H}(\mathbf{A})$ is left nilpotent. This simultaneously proves that if $\mathbf{L} \in \mathcal{K}$ and $\mathbf{L}$ is a 0,1 -sublattice of Con $\mathbf{A}$, then $\mathbf{H}(\mathbf{A})$ is left nilpotent.

Theorem 3.7 If $\mathbf{A}$ is a finite algebra and

$$
\bigvee\{\beta \in \operatorname{Con} \mathbf{A} \mid \bigvee\{\gamma \mid \gamma \wedge \beta=0\}=1\}=1
$$

then $\mathbf{H}(\mathbf{A})$ is left nilpotent.

Proof: Let $S=\{\beta \in$ Con $\mathbf{A} \mid \bigvee\{\gamma \mid \gamma \wedge \beta=0\}=1\}$. For each $\beta \in S$ let $S_{\beta}=$ $\{\gamma \in$ Con $\mathbf{A} \mid \gamma \wedge \beta=0\}$. Our assumption on Con $\mathbf{A}$ is that $\bigvee S=1$ and, for every $\beta \in S$, $\bigvee S_{\beta}=1$. For all $\beta \in S$ and all $\gamma \in S_{\beta}$ we have $\gamma \wedge \beta=0$, so $C(\gamma, \beta ; 0)$ holds by Theorem $2.1(v)$. Hence, $C\left(\vee S_{\beta}, \beta ; 0\right)$ or $C(1, \beta ; 0)$ holds for each $\beta \in S$. This proves that $[1, \beta]=0$ for each $\beta \in S$.

Now choose an arbitrary $\delta \prec \theta$ in Con A. For each $\beta \in S$ we have $(\beta]^{2}=[\beta, \beta] \leq[1, \beta]$ $=0$ and $[\theta, \beta] \leq[1, \beta]=0 \leq \delta$. Theorem 3.4 applies to show that $C(\beta, \theta ; \delta)$ holds for all $\beta \in S$. Therefore, $C(\bigvee S, \theta ; \delta)$ or $C(1, \theta ; \delta)$ holds for all $\delta \prec \theta$ in Con $\mathbf{A}$. This is equivalent to saying that $\mathbf{H}(\mathrm{A})$ is left nilpotent.

\section{Coherence and Regularity}

The results of the first half of this section are presented to show that left nilpotence is well-behaved for coherent algebras in the sense that homomorphic images of left nilpotent, coherent algebras are again left nilpotent. We will find that, although coherence is a somewhat complicated notion, many interesting sorts of algebras are coherent. In Lemma 4.9 we see that E-minimal algebras are coherent. Corollary 4.4 and Theorem 3.7 prove that certain congruence lattices force coherence. For example, any finite algebra whose congruence lattice satisfies

$$
\bigvee\{\beta \mid \bigvee\{\gamma \mid \gamma \wedge \beta=0\}=1\}=1
$$

is coherent. It is fairly easy to prove directly that any abelian algebra is coherent, too. Or, one can derive it from either Theorem 4.5 or the combination of Lemma 4.13 and Theorem 4.20 . 
The second half of this section deals with the concept of regularity. Regularity is stronger than coherence and consequently the assumption that an algebra is regular is strong enough to imply that left nilpotence behaves well with respect to homomorphisms. Further, it is just strong enough to imply that the notions of right and left nilpotence agree. We will show that finite abelian algebras are regular and deduce from this that every homomorphic image of a finite abelian algebra is left and right nilpotent.

Definition 4.1 Let $\langle\delta, \theta\rangle$ be a tame quotient of the finite algebra $\mathbf{A}$ and let $\mathrm{T}=\mathrm{T}_{\mathbf{A}}(\delta, \theta)$ be the set of $\langle\delta, \theta\rangle$-traces of $\mathbf{A}$. We say that $\langle\delta, \theta\rangle$ is $\beta$-coherent if the following implication holds:

$$
\left(\&_{N \in \mathrm{T}} C\left(\beta, N^{2} ; \delta\right)\right) \rightarrow C(\beta, \theta ; \delta) .
$$

If every prime quotient of $\mathbf{A}$ is 1-coherent we say that $\mathbf{A}$ is coherent.

Lemma 4.2 If $\langle\delta, \theta\rangle$ is a tame quotient of the finite algebra $\mathbf{A}$ and $\beta$ is a congruence on $\mathbf{A}$, then $\langle\delta, \theta\rangle$ fails to be $\beta$-coherent if and only if $\operatorname{typ}(\delta, \theta)=\mathbf{1}$ and

(i) $C\left(\beta, N^{2} ; \delta\right)$ holds for every $\langle\delta, \theta\rangle$-trace $N$,

(ii) there exists $p(x, \bar{y}) \in \mathrm{Pol}_{n+1} \mathbf{A}$ which satisfies $p(p(x, \bar{y}), \bar{y})=p(x, \bar{y})\left(\stackrel{\text { def }}{=} p_{\bar{y}}(x)\right)$,

(iii) there exist pairs $\left(a_{i}, b_{i}\right) \in \beta$ for $i<n$,

(iv) there exist $\langle\delta, \theta\rangle$-traces $M$ and $M^{\prime}$ contained in the same $\theta$-class such that

$(v) p_{\bar{a}}(x)$ and $p_{\bar{b}}(x)$ are polynomial isomorphisms of $M^{\prime}$ onto $M$ and

(vi) $\left(p_{\bar{a}}(v), p_{\bar{b}}(v)\right) \notin \delta$ for some $v \in M^{\prime}$.

Proof: If $\operatorname{typ}(\delta, \theta) \neq \mathbf{1}$, then all $\langle\delta, \theta\rangle$-traces are polynomially isomorphic. Hence for types other than $\mathbf{1}$, coherence simply means

$$
C\left(\beta, N^{2} ; \delta\right) \rightarrow C(\beta, \theta ; \delta)
$$

for some (any) trace $N$. We will now show that this implication holds whenever $\operatorname{typ}(\delta, \theta) \neq$ 1.

Assume first that $\operatorname{typ}(\delta, \theta) \in\{\mathbf{3}, \mathbf{4}, \mathbf{5}\}$, that $C\left(\beta, N^{2} ; \delta\right)$ for some trace $N \subseteq U$ where $U$ is a $\langle\delta, \theta\rangle$-minimal set and that $\neg C(\beta, \theta ; \delta)$. Because $\operatorname{typ}(\delta, \theta) \in\{\mathbf{3}, \mathbf{4}, \mathbf{5}\}$, we have

$$
\left.\left.\left.C(\beta, \theta ; \delta) \leftrightarrow \beta \vee \delta \nsupseteq \theta \leftrightarrow \beta\right|_{U} \vee \delta\right|_{U} \nsucceq \theta\right|_{U} .
$$

Since we are assuming that $C(\beta, \theta ; \delta)$ fails, we conclude that $\left.\left.\beta\right|_{U} \vee \delta\right|_{U} \geq\left.\theta\right|_{U}$. As is described in Lemma 4.15 of [4], $N=I \cup O=\{1\} \cup O$. Choose $u \in O$ and assume for a moment that there is an $a \in U-\{1\}$ such that $(1, a) \in \beta$. If $p(x, y)$ is the pseudo-meet operation of $U$, then

$$
p(a, 1)=a \delta p(a, u)
$$

but

$$
p(1,1)=1 \theta-\delta u=p(1, u)
$$


This is a failure of $C\left(\beta, N^{2} ; \delta\right)$ which is contrary to our assumptions. Hence $1 /\left.\beta\right|_{U}=\{1\}=$ $1 /\left.\delta\right|_{U}$. But our earlier conclusion that $\left.\left.\beta\right|_{U} \vee \delta\right|_{U} \geq\left.\theta\right|_{U}$ now shows that

$$
\{1\}=1 /\left(\left.\left.\beta\right|_{U} \vee \delta\right|_{U}\right) \supseteq 1 /\left.\theta\right|_{U}=N
$$

which is false. It follows that $C\left(\beta, N^{2} ; \delta\right)$ and $\neg C(\beta, \theta ; \delta)$ are mutually exclusive conditions when $\operatorname{typ}(\delta, \theta) \in\{\mathbf{3}, \mathbf{4}, \mathbf{5}\}$ and so

$$
C\left(\beta, N^{2} ; \delta\right) \rightarrow C(\beta, \theta ; \delta)
$$

Assume now that $\operatorname{typ}(\delta, \theta)=\mathbf{2}$, that $C\left(\beta, N^{2} ; \delta\right)$ holds for some trace $N \subseteq U$ where $U$ is a $\langle\delta, \theta\rangle$-minimal set and that $\neg C(\beta, \theta ; \delta)$. If $\left.\beta\right|_{U} \nsubseteq B^{2} \cup T^{2}$, then choose $a \in T$ and $u \in B$ such that $(a, u) \in \beta$. If $C\left(\beta, N^{2} ; \delta\right)$ holds for some trace $N$, then it holds for all traces since they are polynomially isomorphic. We may assume that $N$ is the trace of $U$ containing $u$. Choose $v \in N$ with $(u, v) \notin \delta$. Then, if $d(x, y, z)$ is the pseudo-malcev polynomial of $U$, we have

$$
d(a, u, v) \delta a=d(a, v, v)
$$

and

$$
d(u, u, v)=v \theta-\delta u=d(u, v, v)
$$

which is a failure of $C\left(\beta, N^{2} ; \delta\right)$, a contradiction. We conclude that $\left.\beta\right|_{U} \subseteq B^{2} \cup T^{2}$. By Lemma 3.2, we have $C(\beta, \theta ; \delta) \leftrightarrow C(\theta, \beta ; \delta)$. From our remarks in Section 2 and the fact that $\theta=\operatorname{Cg}\left(N^{2} \cup \delta\right)$, we get that $C(\theta, \beta ; \delta) \leftrightarrow C\left(N^{2}, \beta ; \delta\right)$. Hence we are in a situation where $C\left(N^{2}, \beta ; \delta\right)$ fails. This means we can find a polynomial $p(x, \bar{y})$ and elements $(u, v) \in N^{2}$, $\left(a_{i}, b_{i}\right) \in \beta$ such that

$$
p(u, \bar{a}) \delta p(u, \bar{b})
$$

while

$$
p(v, \bar{a}) \theta-\delta p(v, \bar{b}) .
$$

We may assume that $p\left(A, A^{n}\right) \subseteq U$ and, since all four of the elements just mentioned are in a $\theta_{U}$-class which is not a $\left.\delta\right|_{U}$-class, that all four of these elements belong to $N$. As in Lemma 3.2 , if we let $p^{\prime}(x, \bar{y})=d(p(x, \bar{y}), p(x, \bar{a}), p(v, \bar{a}))$ where $d$ is the pseudo-malcev polynomial of $U$, then we get

$$
p^{\prime}(u, \bar{a})=p^{\prime}(v, \bar{a})
$$

while

$$
p^{\prime}(u, \bar{b}) \theta-\delta p^{\prime}(v, \bar{b})
$$

Changing as to $b$ s one at a time, we find that there is an $i$ such that

$$
p^{\prime}\left(u, b_{0}, \ldots, b_{i-1}, a_{i}, \ldots, a_{n-1}\right) \delta p^{\prime}\left(v, b_{0}, \ldots, b_{i-1}, a_{i}, \ldots, a_{n-1}\right)
$$

while

$$
p^{\prime}\left(u, b_{0}, \ldots, b_{i}, a_{i+1}, \ldots, a_{n-1}\right) \theta-\delta p^{\prime}\left(v, b_{0}, \ldots, b_{i}, a_{i+1}, \ldots, a_{n-1}\right) .
$$

Let $p^{\prime \prime}(x, y)=p^{\prime}\left(x, b_{0}, \ldots, b_{i-1}, y, a_{i+1}, \ldots, a_{n-1}\right)$. With this polynomial we have

$$
p^{\prime \prime}\left(u, a_{i}\right) \delta p^{\prime \prime}\left(v, a_{i}\right)
$$


while

$$
p^{\prime \prime}\left(u, b_{i}\right) \theta-\delta p^{\prime \prime}\left(v, b_{i}\right)
$$

which is a failure of $C\left(\beta, N^{2} ; \delta\right)$. This is contrary to our assumptions, so

$$
C\left(\beta, N^{2} ; \delta\right) \rightarrow C(\beta, \theta ; \delta)
$$

for any $\langle\delta, \theta\rangle$-trace $N$ when $\operatorname{typ}(\delta, \theta)=\mathbf{2}$.

Now we know that if $\langle\delta, \theta\rangle$ is not $\beta$-coherent, then $\operatorname{typ}(\delta, \theta)=\mathbf{1}$. Further, since $\langle\delta, \theta\rangle$ is not $\beta$-coherent, we know that $C\left(\beta, N^{2} ; \delta\right)$ for all $N \in \mathrm{T}_{\mathbf{A}}(\delta, \theta)$ while $\neg C(\beta, \theta ; \delta)$ holds. If we look back to the proof of Lemma 3.3 we find that after the proof of Claim 1 we broke the argument into 2 cases. These cases were precisely the cases: $(1) C(\beta, \theta ; \delta)$ fails because $\neg C\left(\beta, N^{2} ; \delta\right)$ for some $\langle\delta, \theta\rangle$-trace $N$ and $(2) C\left(\beta, N^{2} ; \delta\right)$ for all $\langle\delta, \theta\rangle$-traces $N$, but $C(\beta, \theta ; \delta)$ fails anyway. That is, a type 1 tame quotient fails to be $\beta$-coherent if and only if it is a quotient of the kind described in Case 2 of the proof of Lemma 3.3. In the proof of Lemma 3.3 we proved that such quotients are just those for which the conditions $(i)-(v i)$ of this lemma hold. This finishes the proof.

The proof of this lemma shows that a tame quotient is $\beta$-coherent if and only if it is not a type 1 quotient of the sort decribed in Case 2 of the proof Lemma 3.3. We leave it to the reader to verify that if $\langle\delta, \theta\rangle$ is a $\beta$-coherent tame quotient of the finite algebra $\mathbf{A}$ and $\alpha \leq \beta \wedge \delta$, then $\langle\delta / \alpha, \theta / \alpha\rangle$ is a $\beta / \alpha$-coherent tame quotient of the algebra $\mathbf{A} / \alpha$.

Theorem 4.3 Assume that $\mathbf{A}$ is a finite algebra and every prime quotient of $\mathbf{A}$ is $\beta$ coherent. Then the following conditions are equivalent:

(i) $\beta$ is left nilpotent.

(ii) Ranges of idempotent $\beta$-twins have the same cardinality.

$(\text { ii })^{\prime}$ If $(a, b) \in \beta$ and $e(x, y) \in \mathrm{Pol}_{2} \mathbf{A}$ satisfies $e(e(x, y), y)=e(x, y)$, then the ranges of $e(x, a)$ and $e(x, b)$ have the same cardinality.

(iii) $C(\beta, \theta ; \delta)$ holds for all $\delta \prec \theta$ in Con $\mathbf{A}$.

Proof: Assume that $\beta$ is left nilpotent, but that $p_{\bar{a}}(x)$ and $p_{\bar{b}}(x)$ are idempotent $\beta$ twins and $\left|p_{\bar{a}}(A)\right|<\left|p_{\bar{b}}(A)\right|$. If we replace $p_{\bar{y}}(x)$ by $q_{\bar{y}}(x)=p_{\bar{b}}\left(p_{\bar{y}}(x)\right)$ and iterate until the polynomial $r_{\bar{y}}(x)=q_{\bar{y}}\left(\cdots q_{\bar{y}}(x) \cdots\right)$ satisfies $r_{\bar{y}}\left(r_{\bar{y}}(x)\right)=r_{\bar{y}}(x)$, we find that there are idempotent $\beta$-twins, $r_{\bar{a}}(x)$ and $r_{\bar{b}}(x)$, for which $r_{\bar{a}}(A) \subset r_{\bar{b}}(A)=p_{\bar{b}}(A)$. This implies that $r_{\bar{b}}\left(r_{\bar{a}}(x)\right)=r_{\bar{a}}(x)$. Choose $u \in r_{\bar{b}}(A)-r_{\bar{a}}(A)$. Since $r_{\bar{a}}(u) \beta r_{\bar{b}}(u)=u\left(\right.$ or $\left.\left(r_{\bar{a}}(u), u\right) \in(\beta]^{1}\right)$, $\left(a_{i}, b_{i}\right) \in \beta$ and

$$
r(r(u, \bar{a}), \bar{a})=r(u, \bar{a})
$$

we get

$$
r_{\bar{a}}(u)=r(r(u, \bar{a}), \bar{b})(\beta]^{2} r(u, \bar{b})=u .
$$

Repeating this argument proves that $\left(r_{\bar{a}}(u), u\right) \in(\beta]^{k}$ for all $k$. $\beta$ is left nilpotent, so we must have $r_{\bar{a}}(u)=u$. But this is impossible, since we chose $u \notin r_{\bar{a}}(A)$. 
Clearly $(i i)^{\prime}$ is a special case of $(i i)$. In fact, these properties are equivalent. To see that $(i i)^{\prime}$ implies $(i i)$, assume that $(i i)^{\prime}$ holds, that $e(x, \bar{y}) \in \operatorname{Pol}_{n+1} \mathbf{A},\left(a_{i}, b_{i}\right) \in \beta$ for $i<n$, and that $e(x, \bar{a}), e(x, \bar{b}) \in E(\mathbf{A})$. We must show that $e(x, \bar{a})$ and $e(x, \bar{b})$ have ranges of the same cardinality. Let $e^{\prime}(x, \bar{y})$ be a first-variable iterate of $e(x, \bar{y})$ which satisfies $e^{\prime}\left(e^{\prime}(x, \bar{y}), \bar{y}\right)=$ $e^{\prime}(x, \bar{y})$. Then $e^{\prime}(x, \bar{a})=e(x, \bar{a})$ and $e^{\prime}(x, \bar{b})=e(x, \bar{b})$. It suffices to show that $e^{\prime}(x, \bar{a})$ and $e^{\prime}(x, \bar{b})$ have ranges of the same cardinality. Now define

$$
e_{i}(x, y)=e^{\prime}\left(x, b_{0}, \ldots, b_{i-1}, y, a_{i+1}, \ldots, a_{n-1}\right) .
$$

From the choice of $e^{\prime}$ we have $e_{i}\left(e_{i}(x, y), y\right)=e_{i}(x, y)$. Since $\left(a_{i}, b_{i}\right) \in \beta$ and property $(i i)^{\prime}$ holds, we get that $e_{i}\left(x, a_{i}\right)$ and $e_{i}\left(x, b_{i}\right)$ have ranges of the same cardinality. But $e_{i}\left(x, b_{i}\right)=$ $e_{i+1}\left(x, a_{i+1}\right)$. Hence $e_{0}\left(x, a_{0}\right)$ has a range of the same cardinality as $e_{0}\left(x, b_{0}\right)=e_{1}\left(x, a_{1}\right)$ which has a range of the same cardinality as $e_{1}\left(x, b_{1}\right)=e_{2}\left(x, a_{2}\right)$, etc. We conclude that $e_{0}\left(x, a_{0}\right)\left(=e^{\prime}(x, \bar{a})=e(x, \bar{a})\right)$ has range of the same cardinality as $e_{n-1}\left(x, b_{n-1}\right)\left(=e^{\prime}(x, \bar{b})\right.$ $=e(x, \bar{b}))$.

For $($ ii $) \rightarrow($ iii $)$ assume that $C(\beta, \theta ; \delta)$ fails for some $\delta \prec \theta$. If we choose $\theta$ maximal for this failure we get that $(\beta, 1]^{k} \leq \theta$ for some $k$, so $(\beta]^{k+1} \leq \theta$ for the same $k$. Let $U$ be a $\langle\delta, \theta\rangle$-minimal set and let $B$ and $T$ denote the body and tail of $U$. By our choices, $\left.\left.(\beta]^{k+1}\right|_{U} \subseteq \theta\right|_{U} \subseteq B^{2} \cup T^{2}$ for some $k$. From here the argument that $(i i) \rightarrow(i i i)$, or more accurately $\neg(i i i) \rightarrow \neg(i i)$, depends upon the type of $\langle\delta, \theta\rangle$. First we will assume that $\operatorname{typ}(\delta, \theta) \in\{\mathbf{3}, \mathbf{4}, \mathbf{5}\}$ and prove that $(i i)$ fails. Looking back to the proof of Lemma 3.1, we find that since $C(\beta, \theta ; \delta)$ fails we must have $\beta \vee \delta \geq \theta$. Let $p(x, y)$ be the pseudo-meet operation of $U$. We have $p(A, A) \subseteq U, p(x, p(x, y))=p(x, y), U$ has an element 1 such that $p(1, x)=x$ on $U, 1 /\left.\delta\right|_{U}=\{1\}$ and $1 /\left.\theta\right|_{U} \neq\{1\}$. (See Lemma 4.15 of [4] for all of these facts.) We cannot have $1 /\left.\beta\right|_{U}=\{1\}$ since $\left.\left.\beta\right|_{U} \vee \delta\right|_{U} \geq\left.\theta\right|_{U}$, so choose $a \in 1 /\left.\beta\right|_{U}-\{1\}$. Now $p(1, x)$ and $p(a, x)$ are idempotent $\beta$-twins with $p(1, A)=U$ while $p(a, A) \subseteq U$ and $1 \notin p(a, A)$. Hence, $|p(a, A)|<|p(1, A)|$ and $(i i)$ fails.

Now suppose that $\operatorname{typ}(\delta, \theta)=\mathbf{2}$. We explained in the last paragraph why $\left.(\beta]^{k+1}\right|_{U} \subseteq$ $B^{2} \cup T^{2}$. Hence, $C(\theta, \beta ; \delta)$ fails, too, by Lemma 3.2. From the first paragraph of the proof of Lemma 3.2 we see that the failure of $C(\theta, \beta ; \delta)$ means that there is a $p^{\prime}(x, \bar{y}) \in \operatorname{Pol}_{n+1} \mathbf{A}$, $\left.(u, v) \in \theta\right|_{U}-\left.\delta\right|_{U}$ and $\bar{a}, \bar{b} \in A^{n}$ such that $p\left(a, A^{n}\right) \subseteq U$ and

$$
p^{\prime}(u, \bar{a})=p^{\prime}(v, \bar{a})
$$

and

$$
p^{\prime}(u, \bar{b}) \theta-\delta p^{\prime}(v, \bar{b}) .
$$

Hence $p^{\prime}{ }_{\bar{a}}(x)$ and $p^{\prime} \bar{b}(x)$ are $\beta$-twins where the second is a permutation of $U$, but the first is not. If $r_{\bar{y}}(x)$ is an iterate of $p_{\bar{y}}^{\prime}(x)$ which is idempotent for $\bar{y} \in\{\bar{a}, \bar{b}\}$, then $r_{\bar{a}}(x)$ and $r_{\bar{b}}(x)$ are idempotent $\beta$-twins with $r_{\bar{a}}(A)$ a proper subset of $r_{\bar{b}}(A)=U$. Again $(i i)$ fails.

Finally assume that $\operatorname{typ}(\delta, \theta)=\mathbf{1}$. Since $\left.(\beta]^{k+1}\right|_{U} \subseteq B^{2} \cup T^{2}$, all the arguments from the proof of Lemma 3.3 hold. Specifically, there are two ways that $C(\beta, \theta ; \delta)$ could fail. They are described as Case 1 and Case 2 in the proof of Lemma 3.3. Case 2 cannot occur if $\langle\delta, \theta\rangle$ is $\beta$-coherent, so we only need to consider Case 1 . In this case, we have $r(x, y) \in \mathrm{Pol}_{2} \mathbf{A}$ satisfying $r(A, A) \subseteq U$ and $r_{x}\left(r_{x}(y)\right)=r_{x}(y)$ for all $x, y \in A$ and $(a, b) \in \beta$ such that $\left.r_{a}\left(\left.\theta\right|_{U}\right) \subseteq \delta\right|_{U}$ while $r_{b}(y)=y$ on $U . r_{a}(x)$ and $r_{b}(x)$ are idempotent $\beta$-twins where $r_{a}(A)$ is a proper subset of $r_{b}(A)=U$. This finishes the proof that (ii) implies $(i i i)$. 
If $C(\beta, \theta ; \delta)$ holds for all $\delta \prec \theta$ in Con $\mathbf{A}$ and $(\beta]^{k} \neq 0$, then setting $\theta=(\beta]^{k}$ we get

$$
(\beta]^{k+1}=\left[\beta,(\beta]^{k}\right] \leq \bigwedge\left\{\delta \mid \delta \prec(\beta]^{k}\right\}<(\beta]^{k}
$$

for any value of $k$. The left nilpotence of $\beta$ follows from this, so (iii) implies $(i)$. This proves the theorem.

In Theorem 4.3 we can remove the hypothesis that every prime quotient of $\mathbf{A}$ is $\beta$ coherent and still prove that $(i i i) \rightarrow(i) \rightarrow(i i) \leftrightarrow(i i)^{\prime}$. Alternately, if we strengthen the hypothesis that every type $\mathbf{1}$ prime quotient of $\mathbf{A}$ is $\beta$-coherent to the hypothesis that every type 1 tame quotient is $\beta$-coherent, then the four conditions of Theorem 4.3 are equivalent to $(\text { iii })^{\prime} C(\beta, \theta ; \delta)$ holds for all tame quotients $\langle\delta, \theta\rangle$. With slight changes in the proof of Theorem 4.3 one can prove that if $\langle\delta, \theta\rangle$ is tame and $\beta$-coherent and $\left.(\beta]^{k}\right|_{U} \subseteq B^{2} \cup T^{2}$ for some $k$ and some $\langle\delta, \theta\rangle$-minimal set $U$ with body $B$ and tail $T$, then the conditions

(i) $(\beta, \theta]^{l} \leq \delta$ for some $l$.

(ii) there do not exist idempotent $\beta$-twins $r_{\bar{a}}(x)$ and $r_{\bar{b}}(x)$ such that $r_{\bar{a}}(A) \subset U=r_{\bar{b}}(A)$

(iii) $C(\beta, \theta ; \delta)$ holds

are equivalent.

There is an interesting way to view the condition described in Theorem 4.3 (ii). To any finite algebra $\mathbf{A}$ we can associate the finite semigroup $\mathbf{P}(\mathbf{A})=\left\langle\operatorname{Pol}_{1} \mathbf{A} ; \circ\right\rangle$. For each congruence $\beta \in$ Con $\mathbf{A}$ the $\beta$-twin relation defines a congruence $\hat{\beta} \in \operatorname{Con} \mathbf{P}(\mathbf{A})$. If a pair of idempotent $\beta$-twins have ranges of different cardinalities, then there is a pair $\left(r_{\bar{a}}, r_{\bar{b}}\right) \in \hat{\beta}$ such that $r_{\bar{a}}(A) \subset r_{\bar{b}}(A)$. Let $e=r_{\bar{a}}(x)$ and $f=r_{\bar{b}}(x)$. Now $e \circ e=e=f \circ e$ and $f \circ f=$ $f$. If $e \circ f=e$, then such a pair comprises a 2-element subsemilattice of $\mathbf{P}(\mathbf{A})$. If $e \circ f=$ $g \neq e$, then $g=e \circ f$ is an idempotent $\beta$-twin of $f \circ f=f$ and $g$ has the same range as $e$. Replacing $e$ by $g$ we get that $(f, g) \in \hat{\beta}-0$ and we find (after a short computation) that $f$ and $g$ comprise a 2-element subsemilattice of $\mathbf{P}(\mathbf{A})$. Hence the condition in Theorem 4.3 (ii) can be rephrased as, " $\hat{\beta}$ restricts trivially to any subsemilattice of $\mathbf{P}(\mathbf{A})$."

Corollary 4.4 (Compare with Corollary 4.16) If $\mathbf{A}$ is a finite algebra, then $\mathbf{H}(\mathbf{A})$ is left nilpotent if and only if $\mathbf{A}$ is coherent and left nilpotent.

Proof: $\mathbf{H}(\mathbf{A})$ is left nilpotent if and only if $C(1, \theta ; \delta)$ holds whenever $\delta \prec \theta$ in Con $\mathbf{A}$. We will prove a result stronger than the statement of the corollary: $C(\beta, \theta ; \delta)$ holds whenever $\delta \prec \theta$ in Con $\mathbf{A}$ if and only if $\beta$ is left nilpotent and every prime quotient of $\mathbf{A}$ is $\beta$-coherent. When $\beta=1$ this is the statement of the corollary.

Theorem 4.3 proves that if $\beta$ is left nilpotent and every prime quotient of $\mathbf{A}$ is $\beta$-coherent, then $C(\beta, \theta ; \delta)$ holds for all $\delta \prec \theta$ in Con $\mathbf{A}$. It also proves that, conversely, if $C(\beta, \theta ; \delta)$ holds for all $\delta \prec \theta$ in Con $\mathbf{A}$, then $\beta$ is left nilpotent. What remains to show is that if $C(\beta, \theta ; \delta)$ holds for all $\delta \prec \theta$ in Con $\mathbf{A}$, then every (type $\mathbf{1}$ ) prime quotient of $\mathbf{A}$ is $\beta$-coherent. We leave it to the reader to look back at the statement and proof of Claim 3 of Lemma 3.3. He will find that this claim proves that either of the conditions $[\beta, \theta] \leq \delta$ or $[\theta, \beta] \leq \delta$ imply that 
$\langle\delta, \theta\rangle$ is $\beta$-coherent if $\langle\delta, \theta\rangle$ is tame. (Further, the hypothesis $\left.(\beta]^{k}\right|_{U} \subseteq B^{2} \cup T^{2}$ of Lemma 3.3 is not used in the proof of Claim 3.) Thus,

$$
C(\beta, \theta ; \delta) \rightarrow[\beta, \theta] \leq \delta \rightarrow\langle\delta, \theta\rangle \text { is } \beta \text {-coherent. }
$$

This finishes the proof.

It seems worthwhile to single out one of the comments made in this proof.

Theorem 4.5 If $\mathbf{A}$ is finite and has congruences $\beta, \delta, \theta$ and $\langle\delta, \theta\rangle$ is tame, then either of the conditions $[\theta, \beta] \leq \delta$ or $[\beta, \theta] \leq \delta$ implies that $\langle\delta, \theta\rangle$ is $\beta$-coherent.

The result of Lemma 4.2 is practically a recipe for constructing non-coherent algebras. However, Corollary 4.4 suggests the questions: "Are there left nilpotent, finite algebras which are not coherent?" and, "Are there right nilpotent, coherent, finite algebras for which $\mathbf{H}(\mathbf{A})$ is not right nilpotent?" Let's see that the answer to both questions is yes.

Example 4 First we look at a 14-element algebra $\mathbf{A}$ of similarity type $\langle 2,1\rangle$ on the universe $A=\left\{u, \bar{u}, v, \bar{v}, w, \bar{w}, u^{\prime}, \bar{u}^{\prime}, v^{\prime}, \bar{v}^{\prime}, w^{\prime}, \bar{w}^{\prime}, a, \bar{a}\right\}$ which is left nilpotent but not coherent. By Corollary 4.4 this algebra has a homomorphic image which is not left nilpotent. The binary operation symbol is $q$ and the $q$-table is:

\begin{tabular}{|c|c|c|c|c|c|c|c|c|c|c|c|c|c|c|}
\hline$q$ & $\mathrm{u}$ & $\overline{\mathrm{u}}$ & $\mathrm{V}$ & $v$ & $\mathrm{~W}$ & $\overline{\mathrm{W}}$ & $u^{\prime}$ & $\overline{\mathrm{u}} '$ & $\mathrm{v}^{\prime}$ & $\overline{\overline{\mathrm{v}}^{\prime}}$ & $\mathrm{w}^{\prime}$ & $\overline{\mathrm{w}}^{\prime}$ & $a$ & $\overline{\mathrm{a}}$ \\
\hline $\mathrm{u}$ & $\mathrm{u}$ & $\overline{\mathrm{u}}$ & $\mathrm{V}$ & $\overline{\mathrm{V}}$ & $\mathrm{W}$ & $\overline{\mathrm{W}}$ & $\mathrm{u}$ & $\overline{\mathrm{u}}$ & $\mathrm{V}$ & & $\mathrm{W}$ & $\overline{\mathrm{W}}$ & a & $\overline{\mathrm{a}}$ \\
\hline$\overline{\mathrm{u}}$ & $\mathrm{u}$ & $\overline{\mathrm{u}}$ & $\mathrm{V}$ & $\overline{\mathrm{V}}$ & W & $\overline{\mathrm{W}}$ & $\mathrm{u}$ & $\overline{\mathrm{u}}$ & $\mathrm{V}$ & $\overline{\mathrm{V}}$ & $\mathrm{W}$ & $\overline{\mathrm{W}}$ & $\mathrm{a}$ & $\overline{\mathrm{a}}$ \\
\hline $\mathrm{V}$ & $\mathrm{u}$ & $\overline{\mathrm{u}}$ & $\mathrm{V}$ & $\overline{\mathrm{V}}$ & $\mathrm{W}$ & $\overline{\mathrm{W}}$ & $\mathrm{u}$ & $\overline{\mathrm{u}}$ & $\mathrm{V}$ & $\overline{\mathrm{V}}$ & $\mathrm{W}$ & $\overline{\mathrm{W}}$ & $\mathrm{a}$ & $\overline{\mathrm{a}}$ \\
\hline$\overline{\mathrm{V}}$ & $\mathrm{u}$ & $\overline{\mathrm{u}}$ & $\mathrm{V}$ & $\overline{\mathrm{V}}$ & $\mathrm{W}$ & $\overline{\mathrm{W}}$ & $\mathrm{u}$ & $\overline{\mathrm{u}}$ & $\mathrm{V}$ & $\overline{\mathrm{V}}$ & $\mathrm{W}$ & $\overline{\mathrm{W}}$ & $\mathrm{a}$ & $\overline{\mathrm{a}}$ \\
\hline $\mathrm{W}$ & $\mathrm{u}$ & $\overline{\mathrm{u}}$ & $\mathrm{V}$ & $\overline{\mathrm{V}}$ & $\mathrm{W}$ & $\overline{\mathrm{W}}$ & $\mathrm{u}$ & $\overline{\mathrm{u}}$ & $\mathrm{V}$ & $\overline{\mathrm{V}}$ & $\mathrm{W}$ & $\overline{\mathrm{W}}$ & $\mathrm{a}$ & $\overline{\mathrm{a}}$ \\
\hline$\overline{\overline{\mathrm{W}}}$ & $\mathrm{u}$ & $\overline{\mathrm{u}}$ & $\mathrm{V}$ & $\overline{\mathrm{V}}$ & $\mathrm{W}$ & $\overline{\mathrm{W}}$ & $\mathrm{u}$ & $\overline{\mathrm{u}}$ & $\mathrm{V}$ & $\overline{\mathrm{V}}$ & $\mathrm{W}$ & $\overline{\mathrm{W}}$ & $\mathrm{a}$ & $\overline{\mathrm{a}}$ \\
\hline$u^{\prime}$ & $\mathrm{u}$ & $\overline{\mathrm{u}}$ & $\mathrm{V}$ & $\overline{\mathrm{V}}$ & $\mathrm{W}$ & $\overline{\mathrm{W}}$ & $\mathrm{u}$ & $\overline{\mathrm{u}}$ & $\mathrm{V}$ & $\overline{\mathrm{V}}$ & $\mathrm{W}$ & $\overline{\mathrm{W}}$ & $\mathrm{a}$ & $\overline{\mathrm{a}}$ \\
\hline$\overline{\mathrm{u}} '$ & $\mathrm{u}$ & $\overline{\mathrm{u}}$ & $\mathrm{V}$ & $\overline{\mathrm{V}}$ & $\mathrm{w}$ & $\overline{\mathrm{W}}$ & $\mathrm{u}$ & $\overline{\mathrm{u}}$ & $\mathrm{V}$ & $\overline{\mathrm{V}}$ & $\mathrm{W}$ & $\overline{\mathrm{W}}$ & $\mathrm{a}$ & $\overline{\mathrm{a}}$ \\
\hline$v^{\prime}$ & $\mathrm{u}$ & $\overline{\mathrm{u}}$ & $\mathrm{V}$ & $\overline{\mathrm{V}}$ & $\mathrm{W}$ & $\overline{\mathrm{W}}$ & $\mathrm{u}$ & $\overline{\mathrm{u}}$ & $\mathrm{V}$ & $\overline{\mathrm{V}}$ & $\mathrm{W}$ & $\overline{\mathrm{W}}$ & $\mathrm{a}$ & $\overline{\mathrm{a}}$ \\
\hline$\overline{\overline{\mathrm{v}}}$ & $\mathrm{u}$ & $\overline{\mathrm{u}}$ & $\mathrm{V}$ & $\overline{\mathrm{V}}$ & $\mathrm{W}$ & $\overline{\mathrm{W}}$ & $\mathrm{u}$ & $\overline{\mathrm{u}}$ & $\mathrm{V}$ & $\overline{\overline{\mathrm{V}}}$ & $\mathrm{W}$ & $\overline{\mathrm{W}}$ & $a$ & $\overline{\mathrm{a}}$ \\
\hline w' & $\mathrm{u}$ & $\overline{\mathrm{u}}$ & $\mathrm{V}$ & $\overline{\mathrm{V}}$ & $\mathrm{W}$ & $\overline{\mathrm{W}}$ & $\mathrm{u}$ & $\overline{\mathrm{u}}$ & $\mathrm{V}$ & $\overline{\mathrm{V}}$ & $\mathrm{W}$ & $\overline{\mathrm{W}}$ & $\mathrm{a}$ & $\overline{\mathrm{a}}$ \\
\hline$\overline{\mathrm{W}}^{\prime}$ & $\mathrm{u}$ & $\overline{\mathrm{u}}$ & $\mathrm{V}$ & $\overline{\mathrm{v}}$ & $\mathrm{W}$ & $\overline{\mathrm{W}}$ & $\mathrm{u}$ & $\overline{\mathrm{u}}$ & $\mathrm{V}$ & $\overline{\overline{\mathrm{V}}}$ & $\mathrm{W}$ & $\overline{\mathrm{W}}$ & $\mathrm{a}$ & $\overline{\mathrm{a}}$ \\
\hline $\mathrm{a}$ & $\overline{\mathrm{u}}$ & $\mathrm{u}$ & $\overline{\mathrm{V}}$ & $\mathrm{V}$ & $\overline{\mathrm{W}}$ & $\mathrm{W}$ & $\overline{\mathrm{V}}$ & $\mathrm{V}$ & $\overline{\mathrm{u}}$ & $\mathrm{u}$ & $\overline{\mathrm{W}}$ & $\mathrm{W}$ & $\overline{\mathrm{a}}$ & $\mathrm{a}$ \\
\hline$\overline{\mathrm{a}}$ & $\overline{\mathrm{u}}$ & $\mathrm{u}$ & $\overline{\mathrm{V}}$ & $\mathrm{V}$ & $\overline{\mathrm{W}}$ & $\mathrm{W}$ & $\overline{\mathrm{V}}$ & $\mathrm{V}$ & $\overline{\mathrm{u}}$ & $\mathrm{u}$ & $\overline{\mathrm{W}}$ & W & $\overline{\mathrm{a}}$ & $\mathrm{a}$ \\
\hline
\end{tabular}

The unary operation symbol is $t$ and the $t$-table is:

\begin{tabular}{|c|c|c|c|c|c|c|c|c|c|c|c|c|c|c|}
\hline $\mathrm{t}$ & $\mathrm{u}$ & $\overline{\mathrm{u}}$ & $\mathrm{V}$ & $\overline{\mathrm{V}}$ & $\mathrm{W}$ & $\overline{\mathrm{W}}$ & $u^{\prime}$ & $\overline{\mathrm{u}^{\prime}}$ & $\mathrm{v}^{\prime}$ & $\overline{\mathrm{v}}^{\prime}$ & $\mathrm{w}^{\prime}$ & $\overline{\mathrm{w}}^{\prime}$ & $\mathrm{a}$ & $\overline{\mathrm{a}}$ \\
\hline & $\mathrm{v}^{\prime}$ & $\overline{\mathrm{v}^{\prime}}$ & $\mathrm{w}^{\prime}$ & $\overline{\mathrm{W}}^{\prime}$ & $u^{\prime}$ & $\overline{\mathrm{u}}$ & $\mathrm{v}^{\prime}$ & $\overline{\mathrm{V}}$ & $\mathrm{w}^{\prime}$ & $\overline{\mathrm{W}}^{\prime}$ & $\mathrm{u}^{\prime}$ & $\overline{\mathrm{u}}^{\prime}$ & $\mathrm{a}$ & $\overline{\mathrm{a}}$ \\
\hline
\end{tabular}

We will name certain congruences of $\mathbf{A}$ and give the corresponding partitions of $A$ :

$$
\theta=\operatorname{Cg}^{\mathrm{A}}\left(\left(u, u^{\prime}\right),(u, \bar{u})\right): u \bar{u} v \bar{v} w \bar{w} u^{\prime} \bar{u}^{\prime} v^{\prime} \bar{v}^{\prime} w^{\prime} \bar{w}^{\prime} / a / \bar{a}
$$




$$
\begin{gathered}
\delta=\operatorname{Cg}^{\mathrm{A}}\left(\left(w, w^{\prime}\right),(w, \bar{w})\right): u \bar{u} / v \bar{v} / w \bar{w} w^{\prime} \bar{w}^{\prime} / u^{\prime} \bar{u}^{\prime} / v^{\prime} \bar{v}^{\prime} / a / \bar{a} \\
\psi=\operatorname{Cg}^{\mathrm{A}}\left(\left(u, u^{\prime}\right),\left(\bar{u}, \bar{u}^{\prime}\right)\right): u v w u^{\prime} v^{\prime} w^{\prime} / \bar{u} \bar{v} \bar{w} \bar{u}^{\prime} \bar{v}^{\prime} \bar{w}^{\prime} / a / \bar{a} .
\end{gathered}
$$

It may be verified by hand that $C(1,1 ; \psi)$ and $C(1, \psi ; 0)$ hold. We explain briefly how to do this.

To show that $C(1,1 ; \psi)$ holds, one only needs to verify that the 4-element algebra $\mathbf{B}=$ $\mathbf{A} / \psi$ is abelian. The operation $t$ is interpreted as the identity function on $B$ and the $q$-table for $\mathbf{B}$ is

\begin{tabular}{|c||c|c|c|c|}
\hline $\mathrm{q}$ & $\mathrm{x}$ & $\overline{\mathrm{x}}$ & $\mathrm{a}$ & $\overline{\mathrm{a}}$ \\
\hline \hline $\mathrm{x}$ & $\mathrm{x}$ & $\overline{\mathrm{x}}$ & $\mathrm{a}$ & $\overline{\mathrm{a}}$ \\
\hline$\overline{\mathrm{x}}$ & $\mathrm{x}$ & $\overline{\mathrm{x}}$ & $\mathrm{a}$ & $\overline{\mathrm{a}}$ \\
\hline $\mathrm{a}$ & $\overline{\mathrm{x}}$ & $\mathrm{x}$ & $\overline{\mathrm{a}}$ & $\mathrm{a}$ \\
\hline$\overline{\mathrm{a}}$ & $\overline{\mathrm{x}}$ & $\mathrm{x}$ & $\overline{\mathrm{a}}$ & $\mathrm{a}$ \\
\hline
\end{tabular}

To show that $\mathbf{B}$ is abelian it suffices to exhibit a congruence on $\mathbf{B}^{2}$ which has the diagonal as a congruence block. One such congruence is the one determined by the partition of $B^{2}$ given by:

$(x, x)(\bar{x}, \bar{x})(a, a)(\bar{a}, \bar{a}) /(x, \bar{x})(\bar{x}, x)(a, \bar{a})(\bar{a}, a) /(a, \bar{x})(\bar{a}, x) /(x, \bar{a})(\bar{x}, a) /(a, x)(\bar{a}, \bar{x}) /(x, a)(\bar{x}, \bar{a})$.

We leave it to the reader to verify that this partition determines a congruence on $\mathbf{B}^{2}$.

To prove that $C(1, \psi ; 0)$ one needs to show that, for $\mathbf{C}$ equal to the subalgebra of $\mathbf{A}^{2}$ whose universe is $\psi$, the diagonal $\Delta$ is a block of some congruence. One such congruence is the set $\beta=$

$$
\left\{\langle(x, y),(z, w)\rangle \in C^{2} \mid(x, y)=(z, w) ; x=y \text { and } z=w ;(x, y)=(\bar{z}, \bar{w}) \text {; or } \quad(z, w)=(\bar{x}, \bar{y})\right\} \text {. }
$$

We leave it to the reader to verify that $\beta$ is a congruence on $\mathbf{C}$. For a hint on how to do this, first show that the function $f: \mathbf{A} \rightarrow \mathbf{A}$ which interchanges any $x$ with $\bar{x}$ is an automorphism of $\mathbf{A}$ which satisfies $f(q(x, y))=q(x, f(y))$ and $q(f(x), y))=q(x, y)$. It follows easily from these facts that $\beta$ is compatible with $q$ and $t$.

From $C(1,1 ; \psi)$ and $C(1, \psi ; 0)$ it follows that $\mathbf{A}$ is at most 2-step left nilpotent. On the other hand,

$$
q(u, q(u, u))=u=q\left(u, q\left(u, u^{\prime}\right)\right)
$$

while

$$
q(a, q(a, u))=u \theta-\delta v=q\left(a, q\left(a, u^{\prime}\right)\right) .
$$

Hence $C(1, \theta ; \delta)$ fails. Since $\delta \prec \theta,[1, \theta / \delta]=\theta / \delta$ in Con $\mathbf{A} / \delta$ and so $\mathbf{A} / \delta$ is a homomorphic image of $\mathbf{A}$ which is not left nilpotent. A cannot be coherent or we contradict Corollary 4.4.

One may appeal to Lemma 4.2 to prove that $\langle\delta, \theta\rangle$ is not 1-coherent, and therefore that A is not coherent. For this, the sets $M=\{u, \bar{u}, v, \bar{v}, w, \bar{w}\}$ and $M^{\prime}=\left\{u^{\prime}, \bar{u}^{\prime}, v^{\prime}, \bar{v}^{\prime}, w^{\prime}, \bar{w}^{\prime}\right\}$ are $\langle\delta, \theta\rangle$-traces contained in the same $\theta$-class. If we set $p(x, y)=q(y, q(y, x))$ we find that $p_{y}\left(p_{y}(x)\right)=p_{y}(x)$ and that $p_{a}(x)$ and $p_{u}(x)$ are twin polynomial isomorphisms of $M^{\prime}$ onto $M$. Finally, $\left(p_{u}\left(u^{\prime}\right), p_{a}\left(u^{\prime}\right)\right)=(u, v) \in \theta-\delta$. Using these choices for $M, M^{\prime}$ and $p_{y}(x)$ one can verify that $\langle\delta, \theta\rangle$ is not 1 -coherent. 
Example 5 In this example we show that a finite algebra $\mathbf{A}$ may be coherent and right nilpotent and still have a homomorphic image that is not right nilpotent. Of course, $\mathbf{H}(\mathbf{A})$ must be left nilpotent by Theorem 3.5 and Corollary 4.4. This algebra has universe $A=$ $\left\{u, \bar{u}, v, \bar{v}, w, \bar{w}, u^{\prime}, \bar{u}^{\prime}, v^{\prime}, \bar{v}^{\prime}, w^{\prime}, \bar{w}^{\prime}, a\right\}$ and has similarity type $\langle 2,1,1\rangle$. The binary operation symbol is $q$ and the $q$-table is:

\begin{tabular}{|c|c|c|c|c|c|c|c|c|c|c|c|c|c|}
\hline$q$ & $\mathrm{u}$ & $\overline{\mathrm{u}}$ & $\mathrm{V}$ & $\mathrm{V}$ & $\mathrm{W}$ & $\overline{\mathrm{W}}$ & $\mathrm{u}^{\prime}$ & $\overline{\mathrm{u}}^{\prime}$ & $\mathrm{V}^{\prime}$ & $\overline{\mathrm{v}^{\prime}}$ & $\mathrm{w}^{\prime}$ & $\overline{\overline{\mathrm{W}}^{\prime}}$ & $a$ \\
\hline $\mathrm{u}$ & $\mathrm{u}$ & $\overline{\mathrm{u}}$ & $\mathrm{V}$ & $\overline{\mathrm{V}}$ & $\mathrm{W}$ & $\overline{\mathrm{W}}$ & $\mathrm{u}^{\prime}$ & $\overline{\mathrm{u}}^{\prime}$ & $\mathrm{V}^{\prime}$ & $\overline{\mathrm{V}^{\prime}}$ & $\mathrm{w}^{\prime}$ & $\overline{\mathrm{w}}^{\prime}$ & $\mathrm{u}$ \\
\hline$\overline{\mathrm{u}}$ & $\bar{u}$ & $\overline{\mathrm{u}}$ & $\mathrm{v}$ & $\overline{\mathrm{V}}$ & $\mathrm{W}$ & $\overline{\mathrm{W}}$ & $\mathrm{u}^{\prime}$ & $\overline{\mathrm{u}}^{\prime}$ & $\mathrm{V}^{\prime}$ & $\overline{\mathrm{V}}^{\prime}$ & $\mathrm{w}^{\prime}$ & $\overline{\mathrm{w}}^{\prime}$ & $\mathrm{u}$ \\
\hline $\mathrm{V}$ & $\mathrm{u}$ & $\overline{\mathrm{u}}$ & $\mathrm{V}$ & $\overline{\mathrm{V}}$ & $\mathrm{W}$ & $\overline{\mathrm{W}}$ & $u^{\prime}$ & $\overline{\mathrm{u}}^{\prime}$ & $\mathrm{v}^{\prime}$ & $\overline{\mathrm{v}}^{\prime}$ & $\mathrm{w}^{\prime}$ & $\overline{\mathrm{w}}^{\prime}$ & $\mathrm{u}$ \\
\hline$\overline{\mathrm{V}}$ & $\mathrm{u}$ & $\overline{\mathrm{u}}$ & $\mathrm{V}$ & $\overline{\mathrm{V}}$ & $\mathrm{W}$ & $\overline{\mathrm{W}}$ & $\overline{u^{\prime}}$ & $\overline{\mathrm{u}^{\prime}}$ & $\mathrm{v}^{\prime}$ & $\overline{\overline{\mathrm{V}}^{\prime}}$ & $\mathrm{w}^{\prime}$ & $\overline{\mathrm{w}}^{\prime}$ & $\mathrm{u}$ \\
\hline $\mathrm{W}$ & $\mathrm{u}$ & $\overline{\mathrm{u}}$ & $\mathrm{V}$ & $\overline{\mathrm{V}}$ & $\mathrm{W}$ & $\overline{\mathrm{W}}$ & $u^{\prime}$ & $\overline{\mathrm{u}}^{\prime}$ & $\mathrm{v}^{\prime}$ & $\overline{\mathrm{v}}^{\prime}$ & $\mathrm{w}^{\prime}$ & $\overline{\mathrm{w}}^{\prime}$ & $\mathrm{u}$ \\
\hline$\overline{\mathrm{W}}$ & $\mathrm{u}$ & $\overline{\mathrm{u}}$ & $\mathrm{V}$ & $\overline{\mathrm{V}}$ & $\mathrm{W}$ & $\overline{\mathrm{W}}$ & $\mathrm{u}^{\prime}$ & $\overline{\mathrm{u}^{\prime}}$ & $\mathrm{V}^{\prime}$ & $\overline{\overline{\mathrm{V}}}$ & $\mathrm{w}^{\prime}$ & $\overline{\overline{\mathrm{W}}^{\prime}}$ & $\mathrm{u}$ \\
\hline $\mathrm{u}^{\prime}$ & $\mathrm{u}$ & $\overline{\mathrm{u}}$ & $\mathrm{V}$ & $\overline{\mathrm{V}}$ & $\overline{\mathrm{W}}$ & $\overline{\mathrm{W}}$ & $\mathrm{u}^{\prime}$ & $\overline{\mathrm{u}^{\prime}}$ & $\mathrm{V}^{\prime}$ & $\overline{\overline{\mathrm{v}}^{\prime}}$ & $\mathrm{W}^{\prime}$ & $\overline{\mathrm{w}}^{\prime}$ & $\overline{\mathrm{u}}$ \\
\hline$\overline{\mathrm{u}}$ & $\mathrm{u}$ & $\overline{\mathrm{u}}$ & $\mathrm{V}$ & $\overline{\mathrm{V}}$ & $\mathrm{W}$ & $\overline{\mathrm{W}}$ & $\mathrm{u}^{\prime}$ & $\overline{\mathrm{u}}^{\prime}$ & $\mathrm{V}^{\prime}$ & $\overline{\overline{\mathrm{V}}^{\prime}}$ & $\mathrm{w}^{\prime}$ & $\overline{\mathrm{W}}^{\prime}$ & $\overline{\mathrm{u}}$ \\
\hline $\mathrm{v}^{\prime}$ & $\mathrm{u}$ & $\overline{\mathrm{u}}$ & $\mathrm{V}$ & $\overline{\mathrm{V}}$ & $\mathrm{W}$ & $\overline{\mathrm{W}}$ & $u^{\prime}$ & $\overline{\mathrm{u}}^{\prime}$ & $\mathrm{V}^{\prime}$ & $\overline{\overline{\mathrm{v}}^{\prime}}$ & $\mathrm{w}^{\prime}$ & $\overline{\mathrm{w}}^{\prime}$ & $\overline{\mathrm{u}}$ \\
\hline$\overline{\mathrm{v}}$ & $\mathrm{u}$ & $\overline{\mathrm{u}}$ & $\mathrm{V}$ & $\overline{\mathrm{V}}$ & $\mathrm{W}$ & $\overline{\mathrm{W}}$ & $\mathrm{u}^{\prime}$ & $\overline{\mathrm{u}}^{\prime}$ & $\mathrm{V}^{\prime}$ & $\overline{\mathrm{v}^{\prime}}$ & $\mathrm{w}^{\prime}$ & $\overline{\mathrm{w}}^{\prime}$ & $\overline{\mathrm{u}}$ \\
\hline $\mathrm{w}^{\prime}$ & $\mathrm{u}$ & $\overline{\mathrm{u}}$ & $\mathrm{V}$ & $\overline{\mathrm{V}}$ & $\mathrm{W}$ & $\overline{\mathrm{W}}$ & $\mathrm{u}^{\prime}$ & $\overline{\mathrm{u}^{\prime}}$ & $\mathrm{V}^{\prime}$ & $\overline{\mathrm{v}}^{\prime}$ & $\mathrm{w}^{\prime}$ & $\overline{\mathrm{w}}^{\prime}$ & $\overline{\mathrm{u}}$ \\
\hline$\overline{\overline{\mathrm{w}}}$ & $\mathrm{u}$ & $\overline{\mathrm{u}}$ & $\mathrm{V}$ & $\overline{\bar{V}}$ & $\mathrm{~W}$ & $\overline{\overline{\mathrm{W}}}$ & $\mathrm{u}^{\prime}$ & $\overline{\bar{u}^{\prime}}$ & $\mathrm{v}^{\prime}$ & $\overline{\overline{\mathrm{V}}^{\prime}}$ & $\mathrm{w}^{\prime}$ & $\overline{\mathrm{W}}^{\prime}$ & $\overline{\mathrm{u}}$ \\
\hline $\mathrm{a}$ & $\overline{\mathrm{u}}$ & $\mathrm{u}$ & $\overline{\mathrm{W}}$ & $\mathrm{W}$ & $\overline{\overline{\mathrm{V}}}$ & $\mathrm{V}$ & $\overline{\mathrm{u}^{\prime}}$ & $\mathrm{u}^{\prime}$ & $\overline{\mathrm{w}}^{\prime}$ & $\overline{\mathrm{w}^{\prime}}$ & $\overline{\mathrm{v}}^{\prime}$ & $\mathrm{v}^{\prime}$ & $\mathrm{v}^{\prime}$ \\
\hline
\end{tabular}

The unary operation symbols are $s$ and $t$ and the $s$-table is:

\begin{tabular}{|c|c|c|c|c|c|c|c|c|c|c|c|c|c|}
\hline $\mathrm{S}$ & $\mathrm{u}$ & $\overline{\mathrm{u}}$ & $\mathrm{V}$ & $\overline{\mathrm{V}}$ & $\mathrm{W}$ & $\overline{\mathrm{W}}$ & $\mathrm{u}^{\prime}$ & $\overline{\mathrm{u}^{\prime}}$ & $\mathrm{v}^{\prime}$ & $\overline{\mathrm{v}}^{\prime}$ & $\mathrm{w}^{\prime}$ & $\overline{\mathrm{w}}^{\prime}$ & a \\
\hline & $u^{\prime}$ & $\overline{\mathrm{u}}$ & $\mathrm{v}^{\prime}$ & $\overline{\mathrm{v}}$ & $\mathrm{w}^{\prime}$ & $\overline{\mathrm{w}}^{\prime}$ & $\mathrm{u}$ & $\overline{\mathrm{u}}$ & $\mathrm{V}$ & $\overline{\mathrm{V}}$ & $\mathrm{W}$ & $\overline{\mathrm{W}}$ & $\mathrm{a}$ \\
\hline
\end{tabular}

The $t$-table is:

\begin{tabular}{|c|c|c|c|c|c|c|c|c|c|c|c|c|c|}
\hline $\mathrm{t}$ & $\mathrm{u}$ & $\overline{\mathrm{u}}$ & $\mathrm{V}$ & $\overline{\mathrm{V}}$ & $\mathrm{W}$ & $\overline{\mathrm{W}}$ & $\mathrm{u}^{\prime}$ & $\overline{\mathrm{u}}^{\prime}$ & $\mathrm{V}^{\prime}$ & $\overline{\mathrm{V}}^{\prime}$ & $\mathrm{w}^{\prime}$ & $\overline{\overline{\mathrm{w}}^{\prime}}$ & a \\
\hline & $\mathrm{V}$ & $\overline{\mathrm{V}}$ & $\mathrm{W}$ & $\overline{\mathrm{W}}$ & $\mathrm{u}$ & $\overline{\mathrm{u}}$ & $\mathrm{v}^{\prime}$ & $\overline{\mathrm{V}}^{\prime}$ & $\mathrm{w}^{\prime}$ & $\overline{\mathrm{w}}^{\prime}$ & $\mathrm{u}^{\prime}$ & $\overline{\mathrm{u}}^{\prime}$ & $\mathrm{a}$ \\
\hline
\end{tabular}

The non-trivial proper congruences of $\mathbf{A}$ are:

$$
\begin{gathered}
\alpha=\operatorname{Cg}^{\mathrm{A}}\left(u, v^{\prime}\right): u \bar{u} v \bar{v} w \bar{w} u^{\prime} \bar{u}^{\prime} v^{\prime} \bar{v}^{\prime} w^{\prime} \bar{w}^{\prime} / a \\
\beta=\operatorname{Cg}^{\mathrm{A}}\left(u, u^{\prime}\right): u \bar{u} u^{\prime} \bar{u}^{\prime} / v \bar{v} v^{\prime} \bar{v}^{\prime} / w \bar{w} w^{\prime} \bar{w}^{\prime} / a \\
\gamma=\operatorname{Cg}^{\mathrm{A}}(u, \bar{v}): u v w \bar{u} \bar{v} \bar{w} / u^{\prime} v^{\prime} w^{\prime} \bar{u}^{\prime} \bar{v}^{\prime} \bar{w}^{\prime} / a \\
\epsilon=\operatorname{Cg}^{\mathrm{A}}(u, \bar{u}): u \bar{u} / v \bar{v} / w \bar{w} / u^{\prime} \bar{u}^{\prime} / v^{\prime} \bar{v}^{\prime} / w^{\prime} \bar{w}^{\prime} / a \\
\zeta=\operatorname{Cg}^{\mathrm{A}}(u, v): u v w / \bar{u} \bar{v} \bar{w} / u^{\prime} v^{\prime} w^{\prime} / \bar{u}^{\prime} \bar{v}^{\prime} \bar{w}^{\prime} / a
\end{gathered}
$$

The full congruence lattice of $\mathbf{A}$ is shown in Figure 1. It can be verified by the techniques we've used in the other examples that $C(\theta, 1 ; \delta)$ holds when $\langle\delta, \theta\rangle=\langle\alpha, 1\rangle,\langle\zeta, \alpha\rangle$ or $\langle 0, \zeta\rangle$ while $C(\alpha, 1 ; \beta)$ fails. It follows that $\mathbf{A}$ is at most 3 -step right nilpotent and $\mathbf{A} / \beta$ is not right nilpotent. 


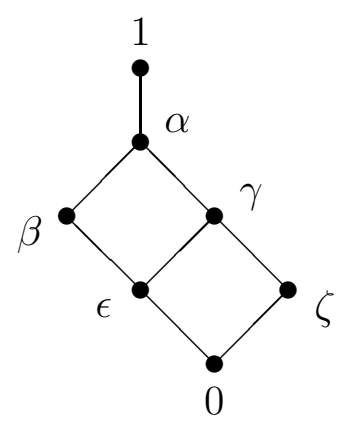

Figure 1: Con A

It is rather tedious to prove that $\mathbf{A}$ is coherent using the definition or Lemma 4.2 . However, there is a short argument which establishes this fact for our algebra. If $\delta \leq \theta$ are congruences on $\mathbf{A}$, we write $\# \theta / \delta$ to denote the supremum of the cardinalities of the sets $\{x / \delta \mid x \in y / \theta\}$ for $y \in A$. Now one can easily calculate from our listing of the congruences on $\mathbf{A}$ that $\# \theta / \delta \leq 3$ whenever $\delta \prec \theta$ in Con $\mathbf{A}$. However, it can be shown that if $\langle\delta, \theta\rangle$ is a type 1 prime (or tame) quotient which is not 1 -coherent, then $\# \theta / \delta \geq 4$. The coherency of A follows from these two observations.

To prove that $\# \theta / \delta \geq 4$ under the assumption that $\langle\delta, \theta\rangle$ is a type $\mathbf{1}$ prime quotient which is not 1-coherent we need only prove it when $\delta=0$. The $\langle 0, \theta\rangle$-traces $M$ and $M^{\prime}$ of Lemma 4.2 must be incomparable under set inclusion because they are polynomially isomorphic and $p_{\bar{a}}(x)$ and $p_{\bar{b}}(x)$ agree on $M$, but they do not agree on $M^{\prime}$. These traces are $\theta$-related, so if $\# \theta / 0 \leq 3$ we would have

$$
3 \geq \# \theta / 0 \geq\left|M \cup M^{\prime}\right| \geq|M|+1 .
$$

Since $|M|>1$, this forces $|M|=2$. The induced algebra $\left.\mathbf{A}\right|_{M}$ must be polynomially equivalent to a 2-element faithful G-set, so $\langle 0, \theta\rangle$ must have cyclic subtype. Such quotients are 1-regular as we explained at the end of Section 2. Now Lemma 4.13 below proves that 1-regular tame quotients are 1-coherent, so we've reached a contradiction. Thus, any finite algebra with $\# \theta / \delta \leq 3$ whenever $\delta \prec \theta$ is coherent.

Definition 4.6 If $\mathbf{A}$ is a finite algebra and $\alpha \in \operatorname{Con} \mathbf{A}$, then write $\mathbf{n i l}^{\mathrm{l}}(\alpha)$ for the largest $\beta \in$ Con $\mathbf{A}$ such that $(\beta / \alpha]^{k}=0$ in Con $\mathbf{A} / \alpha$ for some $k$, if there is a largest such $\beta$. nil ${ }^{1}(\alpha)$ will be called the left nil radical of $\alpha$. Dually, the left nil coradical, nil ${ }_{1}(\alpha)$ is the least $\beta$ such that $(\alpha / \beta]^{k}=0$ in Con $\mathbf{A} / \beta$ for some $k$. The right nil radical and right nil coradical of $\alpha$ are defined similarly and denoted $\mathbf{n i l}^{\mathrm{r}}(\alpha)$ and $\mathbf{n i l}_{\mathrm{r}}(\alpha)$.

Theorem 4.7 Let $\mathbf{A}$ be a coherent finite algebra. The relation $\rho$ on $\mathbf{C o n} \mathbf{A}$ defined by

$$
(\alpha, \beta) \in \rho \longleftrightarrow(\alpha \vee \beta]^{k} \leq \alpha \wedge \beta \text { for some } k
$$

is a tolerance. The corresponding polarity is $\left\langle\operatorname{nil}_{l}(\alpha), \operatorname{nil}^{l}(\alpha)\right\rangle$. In particular, when $\mathbf{A}$ is a coherent finite algebra nil ${ }^{l}(\alpha)$ and $\operatorname{nil}_{l}(\alpha)$ exist for every $\alpha \in$ Con $\mathbf{A}$. As self-maps of Con $\mathbf{A}$, $\mathrm{nil}^{l}(\alpha)$ is an increasing $\wedge$-endomorphism and $\operatorname{nil}_{l}(\alpha)$ is a decreasing $\vee$-endomorphism. 
Proof: We will only prove that $\rho$ is a tolerance on Con $\mathbf{A}$. It is clear from the definitions and the proof of Corollary 4.4 that nil ${ }^{l}(\alpha)$ is the largest $\beta$ such that $(\alpha, \beta) \in \rho$ and $\operatorname{nil}_{l}(\alpha)$ is the smallest $\beta$ such that $(\alpha, \beta) \in \rho$. From here, basic facts about polarities and tolerances (e.g., Lemma 1.2 of [4]) establish all the claims of the theorem.

As defined, $\rho$ is clearly a reflexive and symmetric binary relation on Con $\mathbf{A}$. To show that $\rho$ is compatible with $\wedge$ we must show that if $(\alpha, \beta) \in \rho$ and $\gamma \in$ Con $\mathbf{A}$, then $(\alpha \wedge \gamma, \beta \wedge \gamma) \in \rho$. Since $(\alpha, \beta) \in \rho$, there is some $k$ such that $(\alpha \vee \beta]^{k} \leq \alpha \wedge \beta$. For this same value of $k$ we have

$$
((\alpha \wedge \gamma) \vee(\beta \wedge \gamma)]^{k} \leq(\alpha \vee \beta]^{k} \leq \alpha \wedge \beta
$$

while clearly $((\alpha \wedge \gamma) \vee(\beta \wedge \gamma)]^{k} \leq \gamma$. Thus,

$$
((\alpha \wedge \gamma) \vee(\beta \wedge \gamma)]^{k} \leq \alpha \wedge \beta \wedge \gamma=(\alpha \wedge \gamma) \wedge(\beta \wedge \gamma)
$$

This proves that $(\alpha \wedge \gamma, \beta \wedge \gamma) \in \rho$.

Now we must prove that $\rho$ is compatible with $\vee$ assuming that $\mathbf{A}$ is coherent. Choose $(\alpha, \beta) \in \rho$ and $\gamma \in$ Con $\mathbf{A}$. We know that $(\alpha \vee \beta]^{k} \leq \alpha \wedge \beta$ for some $k$. We must prove that for some $l$ we have

$$
((\alpha \vee \gamma) \vee(\beta \vee \gamma)]^{l} \leq(\alpha \vee \gamma) \wedge(\beta \vee \gamma)
$$

We may replace $\gamma$ by $(\alpha \wedge \beta) \vee \gamma$ without affecting what we must prove, so assume that $\gamma \geq \alpha \wedge \beta$. Now factor by $\alpha \wedge \beta$. This does not affect our hypotheses nor what we must prove, but it does allow us to assume that $\alpha \vee \beta$ is left nilpotent. From Theorem 4.3 $(i) \rightarrow($ iii $)$ we get that $C(\alpha \vee \beta, \theta ; \delta)$ holds whenever $\delta \prec \theta$ in Con $\mathbf{A}$. On the other hand, by Theorem $2.1(v)$ we get that $C(\gamma, \theta ; \delta)$ holds whenever $\gamma \leq \delta \prec \theta$. By Theorem 2.1 (ii) we get $C(\alpha \vee \beta \vee \gamma, \theta ; \delta)$ whenever $\gamma \leq \delta \prec \theta$. Hence there is an $l$ such that

$$
((\alpha \vee \gamma) \vee(\beta \vee \gamma)]^{l}=(\alpha \vee \beta \vee \gamma]^{l} \leq \gamma \leq(\alpha \vee \gamma) \wedge(\beta \vee \gamma)
$$

Thus $(\alpha \vee \gamma, \beta \vee \gamma) \in \rho$ and $\rho$ is a tolerance.

From Theorem 4.7 we see that certain congruence lattices force left nilpotence for coherent algebras. If $\mathbf{A}$ is coherent, Con $\mathbf{A}$ has more than 2 elements and every strictly increasing $\wedge$-endomorphism of Con $\mathbf{A}$ is constant (or equivalently, if every strictly decreasing $\mathbf{V}$-endomorphism is constant), then $\mathbf{A}$ is left nilpotent. The reason is, if Con $\mathbf{A}$ has more than 2 elements and every strictly increasing $\wedge$-endomorphism of Con $\mathbf{A}$ is constant, then Con $\mathbf{A}$ has no prime ideal. But it follows from the theory of solvability outlined in Chapter 7 of [4] and the fact that a finite $\wedge$-semidistributive lattice has a prime ideal that congruence lattices of non-solvable algebras have a prime ideal. Thus $\mathbf{A}$ is at least solvable. Since $\mathbf{A}$ is solvable, $\operatorname{nil}^{l}(x)$ is a strictly increasing $\wedge$-endomorphism of Con $\mathbf{A}$ by Theorem 4.7. Our hypothesis forces $\operatorname{nil}^{l}(0)=1$ and so $\mathbf{A}$ is left nilpotent.

Definition 4.8 (Defn. 2.14 of [4]) A finite algebra is E-minimal if it is non-trivial and every idempotent unary polynomial is constant or the identity.

Another way to say this is that $\mathbf{A}$ is E-minimal if every $p(x) \in \operatorname{Pol}_{1} \mathbf{A}$ is a permutation or has a constant iterate, $q(x)=p(p(\cdots p(x) \cdots))$. We will prove that any solvable E-minimal algebra is left nilpotent although not necessarily right nilpotent. This result includes Lemma 4.36 of [4] which proves that every E-minimal algebra of type $\mathbf{2}$ is nilpotent. (Left and right nilpotency coincide for algebras of type-set $\{\mathbf{2}\}$.) 
Lemma 4.9 If $\mathbf{A}$ is $\langle\delta, \theta\rangle$-minimal, then $\langle\delta, \theta\rangle$ is $\beta$-coherent for every $\beta$. In particular, E-minimal algebras are coherent.

Proof: Referring to the definition of $\beta$-coherence, we see that if $\langle\delta, \theta\rangle$ is not $\beta$-coherent, then the traces $M$ and $M^{\prime}$ cannot be equal since $p_{\bar{a}}(x)=p_{\bar{b}}(x)$ on $M$ while $\left(p_{\bar{a}}(v), p_{\bar{b}}(v)\right) \notin \delta$ for at least one $v \in M^{\prime}$. But if $\mathbf{A}$ is $\langle\delta, \theta\rangle$-minimal, then each $\theta$-class either equals a $\delta$-class or equals a $\langle\delta, \theta\rangle$-trace. It is impossible to find two distinct $\langle\delta, \theta\rangle$-traces in the same $\theta$-class, so $\langle\delta, \theta\rangle$ is $\beta$-coherent for every $\beta$. E-minimal algebras are $\langle\delta, \theta\rangle$-minimal for all $\langle\delta, \theta\rangle$ where $\delta \prec \theta$ by Lemma 4.28 of [4], so every prime quotient of an $E$-minimal algebra is 1-coherent. Hence $E$-minimal algebras are coherent.

Lemma 4.10 Let $\mathbf{A}$ be a solvable E-minimal algebra. If $p(x, \bar{y})$ is an $(n+1)$-ary polynomial of $\mathbf{A}$ and $\bar{a}, \bar{b} \in A^{n}$, then $p(x, \bar{a})$ is a permutation of $A$ if and only if $p(x, \bar{b})$ is.

Proof: Assume that $p(x, \bar{b})$ is a permutation and that $p(x, \bar{a})$ is not. We may iterate $p(x, \bar{y})$ in its first variable and obtain a polynomial $q(x, \bar{y})$ with the properties that $q(x, \bar{b})=$ $x$ while $q(x, \bar{a})$ is constant. Now

$$
q(q(x, \bar{a}), \bar{a})=q(x, \bar{a})
$$

so by changing the last occurrence of $\bar{a}$ to $\bar{b}$ we get

$$
q(x, \bar{a})=q(q(x, \bar{a}), \bar{b})[1,1] q(x, \bar{b})=x .
$$

This holds for all $x$, so

$$
\mathrm{Cg}^{A}(\{(q(x, \bar{a}), x) \mid x \in A\}) \leq[1,1] .
$$

However, the congruence on the left-hand side of this inequality is $1(=A \times A)$ since $q(x, \bar{a})$ is constant. Of course, $[1,1]=1$ is impossible in a non-trivial solvable algebra, so we're done.

The result of Lemma 4.10 implies that non-trivial finite direct powers of a solvable Eminimal algebra are again E-minimal (and have the same type). From the definition of E-minimality one can easily show that any non-trivial homomorphic image or subalgebra of an E-minimal algebra is E-minimal and has the same type. Thus, if $\mathbf{A}$ is a solvable Eminimal algebra, then every non-trivial finite $\mathbf{B} \in \mathbf{V}(\mathbf{A})$ is E-minimal and has the same type. Among other things, this means that an E-minimal algebra of type $\mathbf{2}$ generates a variety with type-set $\{\mathbf{2}\}$. Locally finite varieties with type-set $\{\mathbf{2}\}$ are congruence permutable, so any E-minimal algebra of type $\mathbf{2}$ has a Mal'cev term. A different argument for this pleasant result is outlined in Exercise 13.10 (4) of [4].

Corollary 4.11 A solvable E-minimal algebra is left nilpotent.

Proof: From Lemma 4.9 we know that E-minimal algebras are coherent. To prove that solvable E-minimal algebras are left nilpotent it suffices to verify that Theorem 4.3 ( $i i)$ holds. We must show that ranges of idempotent 1-twins have the same cardinality. But since the idempotent unary polynomials of an E-minimal algebra are constant or the identity, this just 
means that we must show that idempotent 1-twins of a solvable E-minimal algebra are both constant or both the identity. This is proved by Lemma 4.10.

The algebras of Examples 1 and 2 are E-minimal of type 1. These examples serve to show that E-minimal algebras of type $\mathbf{1}$ need not be right nilpotent.

Now we proceed to investigate regular tame quotients in finite algebras. We will find that if $\mathbf{H}(\mathbf{A})$ is left nilpotent and $\mathbf{A}$ is regular, then $\mathbf{H}(\mathbf{A})$ is right nilpotent. The converse follows easily from Theorem 3.5 and the definition of regularity which we reproduce now.

Definition 4.12 Let $\mathbf{A}$ be a finite algebra with a tame quotient $\langle\delta, \theta\rangle$ of type $\mathbf{1}$. If $N$ is a $\langle\delta, \theta\rangle$-trace, let $H_{N, \beta}$ be the group of unary polynomials of $\left.\mathbf{A}\right|_{N}$ consisting of those $\beta$-twins of the identity which are permutations of $N$. If, for all $\langle\delta, \theta\rangle$-traces $N, H_{N, \beta}$ acts regularly on $N$ modulo $\delta$ (meaning that if for some $u \in N$ and $p(x) \in H_{N, \beta}$ we have $p(u) \delta u$, then $p(x) \delta x$ on $N)$ we say that $\langle\delta, \theta\rangle$ is $\beta$-regular. If all type $\mathbf{1}$ prime quotients of $\mathbf{A}$ are 1-regular, we say that $\mathbf{A}$ is regular.

We leave it to the reader to show that if $\alpha \leq \beta \wedge \delta$ and $\langle\delta, \theta\rangle$ is a $\beta$-regular tame quotient of $\mathbf{A}$, then $\langle\delta / \alpha, \theta / \alpha\rangle$ is a $\beta / \alpha$-regular tame quotient of $\mathbf{A} / \alpha$. In particular, homomorphic images of regular algebras are regular.

Lemma 4.13 If $\langle\delta, \theta\rangle$ is a $\beta$-regular tame quotient of type $\mathbf{1}$ of the finite algebra $\mathbf{A}$, then $\langle\delta, \theta\rangle$ is $\beta$-coherent.

Proof: Assume that $\langle\delta, \theta\rangle$ is not $\beta$-coherent. Then $(i) C\left(\beta, N^{2} ; \delta\right)$ holds for every $\langle\delta, \theta\rangle$ trace $N$ and for some $n$ we can find

(ii) $p(x, \bar{y}) \in \mathrm{Pol}_{n+1} \mathbf{A}$ which satisfies $p_{\bar{y}}\left(p_{\bar{y}}(x)\right)=p_{\bar{y}}(x)$

(iii) $\left(a_{i}, b_{i}\right) \in \beta$ for $i<n$,

(iv) $M$ and $M^{\prime}:\langle\delta, \theta\rangle$-traces contained in the same $\theta$-class such that

$(v) p_{\bar{a}}(x)$ and $p_{\bar{b}}(x)$ are polynomial isomorphisms of $M^{\prime}$ onto $M$ and

(vi) $\left(p_{\bar{a}}(v), p_{\bar{b}}(v)\right) \in \theta-\delta$ for some $v \in M^{\prime}$.

Without loss of generality we may assume that

(vii) $p_{\bar{a}}(A)=U=p_{\bar{b}}(A)$ where $U$ is a $\langle\delta, \theta\rangle$-minimal set containing $M$.

To see that we may assume (vii), suppose that $(i)-(v i)$ hold and choose some $U \in \mathrm{M}_{\mathbf{A}}(\delta, \theta)$ such that

$$
M \subseteq U \subseteq p_{\bar{a}}(A) .
$$

To see that there is such a $U$, simply choose any $U^{\prime} \in \mathrm{M}_{\mathbf{A}}(\delta, \theta)$ such that $M \subseteq U^{\prime}$. Then set $U$ $=p_{\bar{a}}\left(U^{\prime}\right)$. The fact that $p_{\bar{a}}(M)=M$ implies that $U \in \mathrm{M}_{\mathbf{A}}(\delta, \theta)$ while clearly $M \subseteq U \subseteq p_{\bar{a}}(A)$. Now replace $p_{\bar{y}}(x)$ with $e p_{\bar{y}}(x)$ where $e(x)$ an idempotent unary polynomial whose range is $U$. All of the conditions $(i)-(v i i)$ will be satisfied with $e p_{\bar{y}}(x)$ in place of $p_{\bar{y}}(x)$ except possibly that $e p_{\bar{y}}(x)$ may not be idempotent when $\bar{y} \neq \bar{a}$. However, any iterate of $e p_{\bar{a}}(x)$ which is idempotent for all $\bar{y} \in A^{n}$ can be used to replace $p_{\bar{y}}(x)$ and $(i)-(v i i)$ will hold. 
Choose $u \in M$. Condition ( $v i$ ) says that there is at least one element, $v$, which is $\theta$-related to $u$ and which satisfies

$$
p_{\bar{a}}(v) \theta-\delta p_{\bar{b}}(v) .
$$

The $\theta$-class containing $M$ and $M^{\prime}$ is connected modulo $\delta$ by $\langle\delta, \theta\rangle$-traces, so for any $v^{\prime} \in u / \theta$ we can find a chain $u=y_{0}, \ldots, y_{m}=v^{\prime}$ where each $\left(y_{i}, y_{i+1}\right) \in \delta$ or $\left(y_{i}, y_{i+1}\right) \in N^{2}-\delta$ for some $\langle\delta, \theta\rangle$-trace $N$. Choose such a $v^{\prime}$ so that

$$
p_{\bar{a}}\left(v^{\prime}\right) \theta-\delta p_{\bar{b}}\left(v^{\prime}\right)
$$

and so that there is no element $w \in u / \theta$ satisfying $\left(p_{\bar{a}}(w), p_{\bar{b}}(w)\right) \in \theta-\delta$ which is connected to $u$ by a shorter chain of the same kind. Necessarily, $\left(p_{\bar{a}}\left(y_{i}\right), p_{\bar{b}}\left(y_{i}\right)\right) \in \delta$ for all $i<m$.

We cannot have both $\left(p_{\bar{a}}\left(y_{m-1}\right), p_{\bar{a}}\left(y_{m}\right)\right) \in \delta$ and $\left(p_{\bar{b}}\left(y_{m-1}\right), p_{\bar{b}}\left(y_{m}\right)\right) \in \delta$, for then

$$
p_{\bar{a}}\left(y_{m}\right) \delta p_{\bar{a}}\left(y_{m-1}\right) \delta p_{\bar{b}}\left(y_{m-1}\right) \delta p_{\bar{b}}\left(y_{m}\right)
$$

which contradicts the fact that $\left(p_{\bar{a}}\left(y_{m}\right), p_{\bar{b}}\left(y_{m}\right)\right) \in \theta-\delta$. So assume that $\left(p_{\bar{b}}\left(y_{m-1}\right), p_{\bar{b}}\left(y_{m}\right)\right) \notin$ $\delta$. In particular, $\left(y_{m-1}, y_{m}\right) \notin \delta$ and the last "link" in our chain from $u$ to $v^{\prime}=y_{m}$ comes from a $\langle\delta, \theta\rangle$-trace which we name $T$. Our assumption that $\left(p_{\bar{b}}\left(y_{m-1}\right), p_{\bar{b}}\left(y_{m}\right)\right) \notin \delta$ means that $p_{\bar{b}}\left(T^{2}\right) \nsubseteq \varnothing \delta$. Necessarily, $p_{\bar{b}}(T)$ is a $\langle\delta, \theta\rangle$-trace contained in $\left.p_{\bar{b}}(T / \theta)\right|_{U}=\left.p_{\bar{b}}(u / \theta)\right|_{U}=$ $\left.(u / \theta)\right|_{U}=M$. Therefore, $p_{\bar{b}}(T)=M$. A similar argument proves that $p_{\bar{a}}(T) \subseteq M$. We claim that $p_{\bar{a}}(T)=M$.

To show that $p_{\bar{a}}(T)=M$, we assume otherwise. Let $g(x) \in \mathrm{Pol}_{1} \mathbf{A}$ be a polynomial isomorphism from $M$ to $T$ which is the inverse to $p_{\bar{b}}(x)$. If $q_{\bar{y}}(x)=g p_{\bar{y}}(x)$, then $q_{\bar{a}}(x), q_{\bar{b}}(x)$ are $\beta$-twin members of $\operatorname{Pol}_{1}\left(\left.\mathbf{A}\right|_{T}\right)$ and $q_{\bar{b}}(x)$ is the identity on $T$ while $q_{\bar{a}}(x)$ is not a permutation of $T$. Therefore, $\left.q_{\bar{a}}\left(T^{2}\right) \subseteq \delta\right|_{T}$ and we can find $(s, t) \in T^{2}-\delta$ such that

$$
q_{\bar{a}}(s)=q_{\bar{a}}(t)
$$

while

$$
q_{\bar{b}}(s)=s \theta-\delta t=q_{\bar{b}}(t) .
$$

This is a failure of $C\left(\beta, T^{2} ; \delta\right)$ which contradicts $(i)$.

Hence, $p_{\bar{a}}(T)=M$ and all of the conditions $(i)-(v i i)$ hold with $T$ in place of $M^{\prime}$ except that now we also have that $T$ contains elements $y_{m-1}$ and $y_{m}$ such that $\left(p_{\bar{a}}\left(y_{m-1}\right), p_{\bar{b}}\left(y_{m-1}\right)\right) \in$ $\delta$ while $\left(p_{\bar{a}}\left(y_{m}\right), p_{\bar{b}}\left(y_{m}\right)\right) \in \theta-\delta$. As in the previous paragraph, let $g(x) \in \mathrm{Pol}_{1} \mathbf{A}$ be a polynomial isomorphism from $M$ to $T$ which is the inverse to $p_{\bar{b}}(x): T \rightarrow M$. Then $q_{\bar{b}}(x)=$ $g p_{\bar{b}}(x)=\mathrm{id}_{T}(x)$ while $q_{\bar{a}}(x)=g p_{\bar{a}}(x) \neq \mathrm{id}_{T}(x)$. In fact, $q_{\bar{a}}\left(y_{m-1}\right) \delta q_{\bar{b}}\left(y_{m-1}\right)=y_{m-1}$ while $q_{\bar{a}}\left(y_{m}\right) \theta-\delta q_{\bar{b}}\left(y_{m}\right)=y_{m}$. This shows that there is a $\langle\delta, \theta\rangle$-trace $T$ where the algebra $\left.\mathbf{A}\right|_{T}$ has a $\beta$-twin of the identity, $q_{\bar{a}}(x)$, for which $q_{\bar{a}}\left(y_{m-1}\right) \delta y_{m-1}$ but $q_{\bar{a}}\left(y_{m}\right) \quad \delta y_{m}$. Hence $\langle\delta, \theta\rangle$ is not $\beta$-regular.

The converse of Lemma 4.13 is false. The algebras in Examples 1 and 2 have a 1-coherent prime quotient $\langle 0, \alpha\rangle$ which is not 1-regular.

Lemma 4.14 If $\operatorname{typ}(\delta, \theta)=1$ and $\langle\delta, \theta\rangle$ is $\beta$-regular, then for the conditions listed below $(i) \rightarrow(i i) \rightarrow($ iii $) \leftrightarrow($ iv $)$ holds. 
(i) $C(\beta, \theta ; \delta)$.

(ii) $[\beta, \theta] \leq \delta$.

(iii) $C(\theta, \beta ; \delta)$.

(iv) $[\theta, \beta] \leq \delta$.

If $\left.(\beta]^{k}\right|_{U} \subseteq B^{2} \cup T^{2}$ for some $k$, then all conditions are equivalent.

Proof: The second statement of the lemma follows from the first statement and Lemma 3.3. For the first part of the lemma we only have two implications to prove. We need to show that either $[\beta, \theta] \leq \delta$ or $[\theta, \beta] \leq \delta$ implies $C(\theta, \beta ; \delta)$. We begin by assuming that $C(\theta, \beta ; \delta)$ fails. Recall that $C\left(N^{2}, \beta ; \delta\right)$ also fails for some $\langle\delta, \theta\rangle$-trace. (Otherwise, if $C\left(N^{2}, \beta ; \delta\right)$ holds for all $\langle\delta, \theta\rangle$-traces, then $C(\alpha, \beta ; \delta)$ would hold where $\alpha$ is the congruence generated by $\delta$ and the squares of all $\langle\delta, \theta\rangle$-traces. But this $\alpha$ is just $\theta$.) There must be a polynomial $p(x, \bar{y}) \in \operatorname{Pol}_{n+1} \mathbf{A}$, a $\langle\delta, \theta\rangle$-minimal set $U$ which we may assume contains $N,(u, v) \in N^{2}$ and $\left(a_{i}, b_{i}\right) \in \beta$ where

$$
p(u, \bar{a}) \delta p(u, \bar{b})
$$

but

$$
p(v, \bar{a}) \theta-\delta p(v, \bar{b}) .
$$

We may assume that $p\left(A, A^{n}\right) \subseteq U$. If $(p(u, \bar{a}), p(v, \bar{a})) \in \delta$ and $(p(u, \bar{b}), p(v, \bar{b})) \in \delta$ then we would have

$$
p(v, \bar{a}) \delta p(u, \bar{a}) \delta p(u, \bar{b}) \delta p(v, \bar{b})
$$

which is false. We may assume that $(p(u, \bar{b}), p(v, \bar{b})) \in \theta-\delta$. Hence $\left.p_{\bar{b}}\left(\left.\theta\right|_{U}\right) \nsubseteq \delta\right|_{U}$ and $p_{\bar{b}}(x)$ is a permutation of $U$. By replacing $p_{\bar{y}}(x)$ by $p_{\bar{b}}{ }^{-1}\left(p_{\bar{y}}(x)\right)$ we may assume that $p_{\bar{b}}(x)=x$ on $U$. (Here and later, if $X \subseteq A$ and $g(x) \in \operatorname{Pol}_{1}\left(\left.\mathbf{A}\right|_{X}\right)$ is a permutation of $X$, we will use the notation " $g^{-1}(x)$ " to denote some iterate of $g(x)$ which acts as an inverse to $g(x)$ on $X$.) If $w \in N=v /\left.\theta\right|_{U}$, then

$$
\left.\left.p_{\bar{a}}(w) \theta\right|_{U} p_{\bar{a}}(v) \theta\right|_{U} p_{\bar{b}}(v)=v \in v /\left.\theta\right|_{U}=N,
$$

so $p_{\bar{a}}(N) \subseteq N$. If $p_{\bar{a}}(x)$ were a permutation of $N$, then $\langle\delta, \theta\rangle$ would not be $\beta$-regular. For $p_{\bar{a}}(x) \in \operatorname{Pol}_{1}\left(\left.\mathbf{A}\right|_{N}\right)$ and $p_{\bar{a}}(x)$ is a $\beta$-twin of the identity satisfying $p_{\bar{a}}(u) \delta u$, but $v \in N$ and $p_{\bar{a}}(v) \theta-\delta v$. Hence we must have $\left.p_{\bar{a}}\left(\left.\theta\right|_{U}\right) \subseteq \delta\right|_{U}$. In fact, $p_{\bar{a}}(N) \subseteq p_{\bar{a}}(u) /\left.\delta\right|_{N}=u /\left.\delta\right|_{N}$.

Let $r_{\bar{y}}(x)$ be an idempotent iterate of $p_{\bar{y}}(x)$. Of course, $r_{\bar{b}}(x)=x$ on $U$ and $\left.r_{\bar{a}}\left(\left.\theta\right|_{U}\right) \subseteq \delta\right|_{U}$ and $N$ is closed under both of these polynomials. Further, $v \in N-r_{\bar{a}}(N)$, so if we set $z=r_{\bar{a}}(v)$ we have

$$
r(v, \bar{a})=r_{\bar{a}}(v)=z=r_{\bar{a}}(z)=r(z, \bar{a})
$$

while

$$
r(v, \bar{b})=r_{\bar{b}}(v)=v \neq z=r_{\bar{b}}(z)=r(z, \bar{b}) .
$$

This witnesses the fact that $[\beta, \theta] \not \leq \delta$ and moreover the fact that $\left[\beta, \mathrm{Cg}^{\mathrm{A}}(z, w)\right]=\mathrm{Cg}^{\mathrm{A}}(z, w)$ for some $(v, w) \in \theta-\delta$. Thus, we even have $(\beta, \theta]^{k} \not \leq \delta$ for all $k$. Also,

$$
r(z, \bar{a})=r_{\bar{a}}(z)=z=r_{\bar{b}}(z)=r(z, \bar{b})
$$


while

$$
r(v, \bar{a})=r_{\bar{a}}(v)=z \neq v=r_{\bar{b}}(v)=r(v, \bar{b}),
$$

so $[\theta, \beta] \not \leq \delta$. As before, we have $\left[\mathrm{Cg}^{\mathrm{A}}(z, w), \beta\right]=\mathrm{Cg}^{\mathrm{A}}(z, w)$, so $[\theta, \beta)^{k} \not \leq \delta$ for all $k$.

In conclusion, we have proved more than just $[\beta, \theta] \leq \delta$ or $[\theta, \beta] \leq \delta$ imply $C(\theta, \beta ; \delta)$ when $\langle\delta, \theta\rangle$ is $\beta$-regular. We have shown that if $C(\theta, \beta ; \delta)$ fails when $\langle\delta, \theta\rangle$ is $\beta$-regular, then $\left[\beta, \mathrm{Cg}^{\mathrm{A}}(z, w)\right]=\mathrm{Cg}^{\mathrm{A}}(z, w)=\left[\mathrm{Cg}^{\mathrm{A}}(z, w), \beta\right]$ for some $(z, w) \in \theta-\delta$.

Theorem 4.15 Let $\mathbf{A}$ be a finite algebra with a left nilpotent congruence $\beta$. Suppose that whenever $\delta \prec \theta \leq \beta$ and $\operatorname{typ}(\delta, \theta)=\mathbf{1}$, the quotient $\langle\delta, \theta\rangle$ is $\beta$-regular. Then $\beta$ is right nilpotent.

Proof: This is just like the proof of Theorem 3.5.

Though not proved in in Lemma 4.14, it is clear that any failure of $\beta$-regularity for $\langle\delta, \theta\rangle$ is a special failure of $C(\theta, \beta ; \delta)$. Hence it is true in fact that

$$
C(\theta, \beta ; \delta) \longleftrightarrow[\theta, \beta] \leq \delta \text { and }\langle\delta, \theta\rangle \text { is } \beta \text {-regular. }
$$

In particular, if $\mathbf{H}(\mathbf{A})$ is right nilpotent, then $C(\theta, 1 ; \delta)$ holds for all $\delta \prec \theta$ in Con $\mathbf{A}$. Thus, $\mathbf{A}$ is right nilpotent and regular. Conversely, if $\mathbf{A}$ is right nilpotent and regular, then $\mathbf{A}$ is left nilpotent by Theorem 3.5 and coherent by Lemma 4.13. Hence $\mathbf{H}(\mathbf{A})$ is left nilpotent by Corollary 4.4. But from this and the regularity of $\mathbf{A}$, Theorem 4.15 proves that $\mathbf{H}(\mathbf{A})$ is right nilpotent. We record this as a corollary to Theorem 4.15.

Corollary 4.16 If $\mathbf{A}$ is a finite algebra, then $\mathbf{H}(\mathbf{A})$ is right nilpotent if and only if $\mathbf{A}$ is regular and right nilpotent.

Corollary 4.17 Suppose that a finite algebra $\mathbf{A}$ is regular. Then the right and left nil (co)radicals of any congruence exist and they coincide. In particular, $\mathbf{A}$ is right or left nilpotent if and only if $\mathbf{H}(\mathbf{A})$ is right and left nilpotent.

In [1] it is shown that in any locally finite, congruence semimodular variety the type $\mathbf{1}$ quotients in any finite algebra are actually type $\mathbf{0}$ and therefore any finite algebra in a congruence semimodular variety is regular. From this and Corollary 4.17 we get the following corollary.

Corollary 4.18 If $\mathcal{V}$ is a locally finite, congruence semimodular variety and $\mathbf{A} \in \mathcal{V}$, then a congruence on $\mathbf{A}$ is locally right nilpotent if and only if it is locally left nilpotent.

In 1988, a tame congruence theory workshop was held in Budapest which generated many new questions about finite algebras. The reference [5] is a survey of that workshop. The following problem is posed there:

Problem 3.8 [5] Characterize those algebras that are homomorphic images of finite abelian algebras. 
Every algebra is a homomorphic image of an absolutely free algebra over a sufficiently large generating set and such algebras are abelian. However, except in the case when the similarity type is empty and the generating set is finite, these algebras are infinite. The finiteness assumption in Problem 3.8 introduces mysterious combinatorial aspects that we do not yet fully understand.

The only properties of homomorphic images of finite abelian algebras that I knew in 1988 were that they are finite and solvable. It seemed, to me at least, that a plausible answer to Problem 3.8 was: An algebra is a homomorphic image of a finite abelian algebra iff it is finite and solvable. This turns out to be false. We will prove that if $\mathbf{B}$ is a homomorphic image of a finite abelian algebra $\mathbf{A}$, then $\mathbf{H}(\mathbf{B})$ must be left and right nilpotent. The proof is accomplished by showing that if $\mathbf{A}$ is a finite abelian algebra, then $\mathbf{A}$ is regular. By Lemma 4.13, $\mathbf{A}$ is coherent, so Corollary 4.4 proves that $\mathbf{H}(\mathbf{A})$ is left nilpotent. $\mathbf{H}(\mathbf{A})$ consists of regular algebras, since $\mathbf{A}$ is regular, so Theorem 4.15 proves that $\mathbf{H}(\mathbf{A})$ is right nilpotent, too.

Before embarking on the proof that every finite abelian algebra is regular we must first establish a fact about groups which is needed in the proof. For this lemma $[x, y]=x^{-1} y^{-1} x y$ denotes the commutator of group theory.

Lemma 4.19 Let $G$ be a group, $H<G$ be a maximal subgroup and

$$
M=\bigcap_{\gamma \in G} \gamma^{-1} H \gamma
$$

If $\sigma \in H-M$, then there is a conjugate element $\sigma^{\tau}=\tau^{-1} \sigma \tau$ such that $\left[\sigma, \sigma^{\tau}\right] \notin M$.

Proof: Assume that $\sigma \in H$ and that for every $\tau \in G,\left[\sigma, \sigma^{\tau}\right] \in M$. We need to show that $\sigma \in M$. Let $N$ be the normal subgroup of $G$ generated by $M \cup\{\sigma\}$. Our assumption that $\forall \tau \in G\left(\left[\sigma, \sigma^{\tau}\right] \in M\right)$ implies that $N / M$ is abelian. We will argue that $N \cap H$ is normal in $G$. Then

$$
M \cup\{\sigma\} \subseteq N \cap H=\bigcap_{\gamma \in G} \gamma^{-1}(N \cap H) \gamma \subseteq \bigcap_{\gamma \in G} \gamma^{-1} H \gamma=M
$$

and $\sigma \in M$. Thus, by showing $(N \cap H) \triangleleft G$ we finish the proof.

If $N \subseteq H$, then $N=N \cap H$ and so $(N \cap H) \triangleleft G$. If $N \nsubseteq H$; then, since $H$ is a maximal subgroup of $G, G=N \vee H$. Therefore, it suffices to prove that $(N \cap H) \triangleleft H$ and $(N \cap H) \triangleleft N$. The former follows from the fact that $N \triangleleft G$ while the latter follows from the fact that $N / M$ is abelian and $M<(N \cap H)<N$.

Theorem 4.20 Assume that $\langle\delta, \theta\rangle$ is a type $\mathbf{1}$ prime quotient of the finite algebra $\mathbf{A}, N$ is $a\langle\delta, \theta\rangle$-trace and $\beta \in$ Con $\mathbf{A}$. If

$$
[\beta, \beta] \wedge \theta \leq \delta,
$$

then $\langle\delta, \theta\rangle$ is $\beta$-regular. Consequently, any finite abelian algebra is regular.

Proof: Assume $\langle\delta, \theta\rangle$ is of type $\mathbf{1}$, but not $\beta$-regular. Then there is a $\langle\delta, \theta\rangle$-minimal set $U$ containing a trace $N$ where the unary polynomial permutations of $\left.\mathbf{A}\right|_{N}$ which are $\beta$-twins of the identity do not act regularly modulo $\delta$ on $N$. This means that there is some $n$, a polynomial $p(x, \bar{y}) \in \operatorname{Pol}_{n+1} \mathbf{A}$ and $\bar{a}, \bar{b} \in A^{n},\left(a_{i}, b_{i}\right) \in \beta$ such that 
(i) $p\left(A, A^{n}\right) \subseteq U$,

(ii) $p_{\bar{a}}(x)$ and $p_{\bar{b}}(x)$ are permutations of $N$ with $p_{\bar{a}}(x)=x$ on $N$, and

(iii) there exist $u, v \in N$ such that $p_{\bar{b}}(u) \delta u$ and $p_{\bar{b}}(v) \theta-\delta v$.

Let $G$ denote the group of all unary polynomial permutations of $\left.\mathbf{A}\right|_{N}$ and let $H$ denote the subgroup of all $g(x) \in G$ for which $g(u) \delta u$. Since the elements of $G$ are polynomials of $\left.\mathbf{A}\right|_{N}$ and $\left.\delta\right|_{N}$ is a congruence of this algebra, $G$ acts naturally on $N /\left.\delta\right|_{N}$. This action is primitive and transitive when $\delta \prec \theta$ and $\operatorname{subtyp}(\delta, \theta) \neq \mathbf{0}$ which happens to be the case for us since $\langle\delta, \theta\rangle$ is a prime quotient which is not $\beta$-regular. As $H$ is precisely the stabilizer of $u /\left.\delta\right|_{N}$ under the action of $G$ on $N /\left.\delta\right|_{N}$, we conclude that $H$ is a maximal subgroup of $G$.

By the definition of $H$ and the fact that $p_{\bar{b}}(u) \delta u$ we have $p_{\bar{b}}(x) \in H$. Because $G$ acts transitively on $N /\left.\delta\right|_{N}$ it is possible to find a $\rho(x) \in G$ such that $\rho(u) \delta v$. We are given that $\left(p_{\bar{a}}(v), p_{\bar{b}}(v)\right)=\left(v, p_{\bar{b}}(v)\right) \in \theta-\delta$, so $\left(\rho^{-1} p_{\bar{a}}(v), \rho^{-1} p_{\bar{b}}(v)\right) \in \theta-\delta$ and therefore

$$
\rho^{-1}\left(p_{\bar{b}}(\rho(u))\right) \theta-\delta \rho^{-1}\left(p_{\bar{a}}(\rho(u))\right)=\rho^{-1}(\rho(u))=u .
$$

This means that $\rho^{-1} p_{\bar{b}} \rho(x) \notin H$ or equivalently that $p_{\bar{b}}(x) \notin \rho H \rho^{-1}$. Defining

$$
M=\bigcap_{\gamma \in G} \gamma^{-1} H \gamma=\{\lambda(x) \in G \mid \lambda(x) \delta x \text { on } N\},
$$

we have just shown that $p_{\bar{b}}(x) \in H-M$. Lemma 4.19 proves that there is a $\tau(x) \in G$ such that the unary polynomial $\left[p_{\bar{b}}, p_{\bar{b}}\right](x) \notin M . M$ consists of those elements $\lambda(x) \in G$ satisfying $\lambda(x) \delta x$ on $N$, so there exists a $w \in N$ such that $\left[p_{\bar{b}}, p_{\bar{b}}^{\tau}\right](w) \theta-\delta w$.

Let $r(\bar{y}, \bar{z}) \in \operatorname{Pol}_{2 n} \mathbf{A}$ be the polynomial $\left[p_{\bar{y}}, p_{\bar{z}}^{\tau}\right](w)$. The fact that $p_{\bar{a}}(x)=x$ on $N$ along with $r(\bar{b}, \bar{b})=\left[p_{\bar{b}}, p_{\bar{b}}^{\tau}\right](w) \theta-\delta w$ gives

$$
r(\bar{a}, \bar{a})=w=r(\bar{a}, \bar{b})
$$

while

$$
r(\bar{b}, \bar{a})=w \theta-\delta r(\bar{b}, \bar{b})
$$

These two equations show that

$$
(r(\bar{b}, \bar{a}), r(\bar{b}, \bar{b})) \in([\beta, \beta] \wedge \theta)-\delta
$$

which proves the first assertion of the theorem. The second assertion follows from the first by taking $\beta=1$.

The result of Theorem 4.20 is false if we relax the assumption that $\langle\delta, \theta\rangle$ is a prime quotient to the hypothesis that it is a tame quotient. In the notation of Definition 4.12 and Theorem 4.20: if the group $H_{N, \beta}$ of $\beta$-twins of the identity of $\left.\mathbf{A}\right|_{N} /\left.\delta\right|_{N}$ which are permutations of $N$ acts trivially or transitively on $N /\left.\delta\right|_{N}$, then one can show that this group must also act regularly. But if $\delta \nprec \theta$, one can construct examples where $H_{N, \beta}$ fails to act trivially, transitively or regularly on $N /\left.\delta\right|_{N}$. 
Corollary 4.21 Every homomorphic image of a finite abelian algebra is left and right nilpotent. Any locally finite variety generated by abelian algebras is locally left and locally right nilpotent.

Proof: Every finite abelian algebra is regular by Theorem 4.20. Any homomorphic image is left nilpotent by Corollary 4.4 and Theorem 4.5. Any homomorphic image must also be right nilpotent by Lemma 4.14. If a locally finite variety is generated by abelian algebras, then the finitely generated free algebras are finite and abelian, so the finite algebras in the variety are homomorphic images of finite abelian algebras. Thus the second claim of the corollary follows from the first.

Finite abelian algebras are regular and nilpotent. The property of being finite, regular and nilpotent is preserved under the formation of homomorphic images. Is it plausible that the class of algebras which are finite, regular and nilpotent is precisely the class of homomorphic images of finite abelian algebras? If we consider only those finite algebras that have no prime quotient of type $\mathbf{2}$, the answer to this may be yes; but in general the answer is no. It can be shown, for example, that no finite, nilpotent, nonabelian group is a homomorphic image of any finite abelian algebra. To exhibit another condition that must be satisfied by homomorphic images of finite abelian algebras we introduce the notation $[\alpha, \beta]_{\delta}$ to denote the least congruence $\gamma$ such that $\gamma \geq \delta$ and $C(\alpha, \beta ; \gamma)$ holds. The new condition is the following one:

Theorem 4.22 If $\mathbf{A}$ is a homomorphic image of a finite abelian algebra, then $[1,1]_{\delta} \stackrel{s s}{\sim} \delta$ for all congruences $\delta \in$ Con $\mathbf{A}$.

Proof: It suffices to prove it when $\mathbf{A}$ is abelian since the condition that $\forall \delta \in$ Con $\mathbf{A}$ $\left([1,1]_{\delta} \stackrel{s s}{\sim} \delta\right)$ is inherited by homomorphic images. Therefore, assume that $\mathbf{A}$ is an abelian algebra and that $[1,1]_{\delta} \stackrel{s \&}{\sim} \delta$ for some $\delta$. This means that there must be congruences $\alpha$ and $\beta$ such that $\delta \leq \alpha \prec \beta \leq[1,1]_{\delta}$ with $\operatorname{typ}(\alpha, \beta) \neq 1$. Since $\mathbf{A}$ is abelian we must have $\operatorname{typ}(\alpha, \beta)=\mathbf{2}$. Let $\gamma \geq \alpha$ be a congruence which is maximal with respect to the condition that $\gamma \nsupseteq \beta$. $\gamma$ is strictly meet-irreducible and its unique upper cover is $\theta=\beta \vee \gamma$. The quotients $\langle\alpha, \beta\rangle$ and $\langle\gamma, \theta\rangle$ are perspective, so they have the same type: that type is 2. Temporarily, assume that $[1,1]_{\gamma} \stackrel{s s}{\sim} \gamma$. Then $[1,1]_{\gamma}=\gamma$ since $[1,1]_{\gamma} \geq \gamma$ and the only congruence $\psi \geq \gamma$ which satisfies $\psi \stackrel{s s}{\sim} \gamma$ is $\psi=\gamma$. But $[1,1]_{\gamma}=\gamma$ means that $C(1,1 ; \gamma)$ holds. We also have $C\left(1,1 ;[1,1]_{\delta}\right)$, so by Theorem 2.1 (iii) we get $C\left(1,1 ; \gamma \wedge[1,1]_{\delta}\right)$. The congruence $\gamma \wedge[1,1]_{\delta}$ is $\geq \delta$, so by definition

$$
[1,1]_{\delta} \leq \gamma \wedge[1,1]_{\delta}
$$

and we get $\beta \leq[1,1]_{\delta} \leq \gamma$. We chose $\gamma$ in such a way that $\beta \not \leq \gamma$, so this is false. We

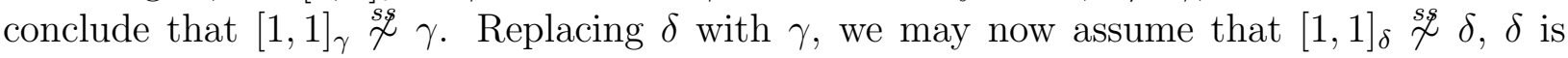
strictly meet-irreducible with unique upper cover $\theta$ and $\operatorname{typ}(\delta, \theta)=\mathbf{2}$.

Let $U$ be a $\langle\delta, \theta\rangle$-minimal set. The tail of $U$ is empty by Lemma 4.27 (iv) of [4] and the fact that $\mathbf{A}$ has no nonabelian prime quotients. Thus $\left.\mathbf{A}\right|_{U}$ is Mal'cev. Now, since we have assumed that $[1,1]_{\delta} \stackrel{s \&}{\not} \delta$, we must have $\neg C(1,1 ; \delta)$. There is an $n$, a polynomial $p(x, \bar{y}) \in \operatorname{Pol}_{n+1} \mathbf{A}, u, v \in A$, and $\bar{a}, \bar{b} \in A^{n}$ such that

$$
p(u, \bar{a}) \delta p(u, \bar{b})
$$


but

$$
c=p(v, \bar{a}) \quad \not p(v, \bar{b})=d .
$$

In particular, from the last displayed line and the fact that $\delta$ is strictly meet-irreducible with cover $\theta$ we get that $\delta \vee \mathrm{Cg}^{\mathrm{A}}(c, d) \geq \theta$. It follows that $\left.\left.\delta\right|_{U} \vee \mathrm{Cg}^{\mathrm{A}}(c, d)\right|_{U} \geq\left.\theta\right|_{U}$. This means that we can find a pair $\left.(r, s) \in \theta\right|_{U}-\left.\delta\right|_{U}$ and a sequence $r=x_{0}, \ldots, x_{n-1}=s$ where, for each $i<n,\left(x_{i}, x_{i+1}\right) \in \delta$ or $\left\{x_{i}, x_{i+1}\right\}=\{g(c), g(d)\}$ where $g(x) \in \operatorname{Pol}_{1} \mathbf{A}$. Let $e(x) \in E(\mathbf{A})$ be an idempotent polynomial whose range is $U$. Then

$$
r=e(r)=e\left(x_{0}\right), \ldots, e\left(x_{n-1}\right)=r(s)=s .
$$

Since $(r, s) \notin \delta$, there is at least one $i$ for which we have $\left\{e\left(x_{i}\right), e\left(x_{i+1}\right)\right\}=\{e g(c), e g(d)\}$ and $(e g(c), e g(d)) \notin \delta$. Thus,

$$
\operatorname{egp}(u, \bar{a}) \delta \operatorname{egp}(u, \bar{b}) \quad \text { and } \quad \operatorname{egp}(v, \bar{a}) \quad \not \operatorname{egp}(v, \bar{b})
$$

and all four of these elements lie in $U$.

If $d(x, y, z)$ is the Mal'cev polynomial of $U$, then define

$$
p^{\prime}(x, \bar{y})=d(\operatorname{egp}(x, \bar{y}), \operatorname{egp}(x, \bar{a}), \operatorname{egp}(v, \bar{a})) .
$$

This is a polynomial of $\mathbf{A}$ for which we have

$$
p^{\prime}(u, \bar{a})=\operatorname{egp}(v, \bar{a})=p^{\prime}(v, \bar{a})
$$

but

$$
p^{\prime}(u, \bar{b}) \delta \operatorname{egp}(v, \bar{a}) \theta-\delta \operatorname{egp}(v, \bar{b})=p^{\prime}(v, \bar{b})
$$

But $\mathbf{A}$ is abelian, so this is impossible. This contradiction shows that the assumption $[1,1]_{\delta} \stackrel{s \&}{\nsim}$ is false and proves the theorem.

The class $\mathcal{C}_{\tau}$ of all finite, nilpotent, regular algebras of a fixed similarity type $\tau$ which satisfy the commutator condition $[1,1]_{\delta} \stackrel{s s}{\sim} \delta$ is closed under the formation of homomorphic images and contains all the finite abelian algebras of type $\tau$, so it is possible that $\mathcal{C}_{\tau}$ is equal to the class of homomorphic images of finite abelian algebras of type $\tau$. Since the class of homomorphic images of finite abelian algebras of type $\tau$ is closed under the formation of subalgebras and finite direct products, any member $\mathbf{A} \in \mathcal{C}_{\tau}$ which is not a homomorphic image of a finite abelian algebra and has minimal cardinality for this property must be subdirectly irreducible. If $\mu$ is the monolith of $\mathbf{A}$, then $\operatorname{typ}(0, \mu) \neq \mathbf{2}$. For, assume that $\operatorname{typ}(0, \mu)=\mathbf{2}$. This means that $\mu \stackrel{s . \mathcal{\sim}}{\sim} 0$. But $\mathbf{A}$ satisfies the commutator condition $[1,1]_{\delta} \stackrel{s s}{\sim} \delta$, so $[1,1]=[1,1]_{0} \stackrel{s s}{\sim} 0$. Therefore $[1,1]=0$ and $\mathbf{A}$ is abelian. This contradicts the fact that $\mathbf{A}$ is not a homomorphic image of a finite abelian algebra. A proof that $\mathcal{C}_{\tau}$ equals the class of homomorphic images of finite abelian algebras of type $\tau$ could be accomplished by showing that $\operatorname{typ}(0, \mu) \neq 1$. We record two variations of the observations made in this paragraph as corollaries to Theorem 4.22.

Corollary 4.23 If $\mathbf{A}$ is a finite subdirectly irreducible algebra with monolith $\mu$ and typ $(0, \mu)$ $=\mathbf{2}$, then $\mathbf{A}$ is a homomorphic image of a finite abelian algebra if and only if $\mathbf{A}$ is abelian. 
Corollary 4.24 If $\mathbf{A}$ is finite and every prime quotient of $\mathbf{A}$ is of type $\mathbf{2}$, then $\mathbf{A}$ is a homomorphic image of a finite abelian algebra if and only if $\mathbf{A}$ is abelian if and only if $\mathbf{H}(\mathbf{A})$ is abelian.

We have reduced Problem 3.8 of [5] to the following problem:

Problem 3 Let $\mathbf{A}$ be a finite subdirectly irreducible algebra with monolith $\mu$ and suppose that $\mathbf{A}$ is regular, nilpotent and that $\operatorname{typ}(0, \mu)=\mathbf{1}$. Suppose also that $\mathbf{A} / \mu$ is a homomorphic image of a finite abelian algebra. Is $\mathbf{A}$ a homomorphic image of a finite abelian algebra? If the answer is "not necessarily," then what additional conditions are needed?

\section{The Weak Term Condition}

The arguments we have seen in the basic lemmas, Lemmas 3.1, 3.2, 3.3 and 4.14, are all of the same form. We argue that, under certain hypotheses, if $\beta$ fails to centralize $\theta$ modulo $\delta$ or $\theta$ fails to centralize $\beta$ modulo $\delta$, then a very special witness to this failure may be exhibited. A witness special enough to show that $[\beta, \theta] \not \leq \delta$ or $[\theta, \beta] \not \leq \delta$. The statement that these special witnesses of $\neg C(\beta, \theta ; \delta)$ and $\neg C(\theta, \beta ; \delta)$ do not exist is a weak kind of term condition. Specifically, suppose that $\mathbf{A}$ is an algebra and $M(\beta, \theta)$ is the set of $2 \times 2$-matrices of the form

$$
\left[\begin{array}{ll}
t\left(\overline{a_{1}}, \overline{b_{1}}\right) & t\left(\overline{a_{1}}, \overline{b_{2}}\right) \\
t\left(\overline{a_{2}}, \overline{b_{1}}\right) & t\left(\overline{a_{2}}, \overline{b_{2}}\right)
\end{array}\right]
$$

where $t$ is a polynomial of $\mathbf{A},\left(a_{1, i}, a_{2, i}\right) \in \beta$ and $\left(b_{1, j}, b_{2, j}\right) \in \theta$. Then we say that $\beta$ centralizes $\theta$ in the sense of the weak term condition if

$$
\left[\begin{array}{ll}
u & u \\
u & v
\end{array}\right] \in M(\beta, \theta) \longrightarrow u=v .
$$

This condition is symmetric in $\beta$ and $\theta$ and weaker than either $C(\beta, \theta ; 0)$ or $C(\theta, \beta ; 0)$. In this paper we have argued that if, say, $\beta$ is left nilpotent and $\langle 0, \theta\rangle$ is tame (and coherent if type $\mathbf{1})$, then $C(\beta, \theta ; 0)$ is equivalent to $C(\theta, \beta ; 0)$ because they are both equivalent to the condition that $\beta$ centralizes $\theta$ in the sense of the weak term condition. In any congruence modular variety $C(\beta, \theta ; 0)$ is equivalent to $C(\theta, \beta ; 0)$ because they are both equivalent to the condition that $\beta$ centralizes $\theta$ in the sense of the weak term condition. The same can be said about any locally finite variety omitting type $\mathbf{1}$ if all type $\mathbf{2}$ minimal sets have empty tails. If $\beta$ centralizes $\theta$ in the sense of the weak term condition but $C(\beta, \theta ; 0)$ fails, we must have a somewhat "bad" algebra. Any algebra with non-symmetric commutator is bad in this sense, so we propose a problem.

Problem 4 Is it true for locally finite varieties that the commutator is symmetric if and only if the term condition agrees with the weak term condition?

Now, call an algebra weakly abelian if the congruence 1 centralizes itself in the sense of the weak term condition. Finite weakly abelian algebras have several familiar properties. 
Theorem 5.1 If $\mathbf{A}$ is a finite weakly abelian algebra, then $\mathbf{A}$ is regular, left and right nilpotent and satisfies the commutator condition $[1,1]_{\delta} \stackrel{s s}{\sim} \delta$.

Proof: If we re-examine the proof of Theorem 4.20 in the case that $\beta=1$ we find that if $\mathbf{A}$ has a prime quotient which is not 1-regular, then we can find $r(\bar{x}, \bar{y}) \in \operatorname{Pol}_{2 n} \mathbf{A}$ and elements $a_{i}, b_{i} \in A, i<n$, such that

$$
\left[\begin{array}{ll}
r(\bar{a}, \bar{a}) & r(\bar{a}, \bar{b}) \\
r(\bar{b}, \bar{a}) & r(\bar{b}, \bar{b})
\end{array}\right]=\left[\begin{array}{ll}
u & u \\
u & v
\end{array}\right] \in M(1,1)
$$

and $u \neq v$. Thus, if $\mathbf{A}$ is weakly abelian, then it is regular. In particular, $\mathbf{A}$ is coherent and we may use the criterion of Theorem 4.3 (ii) to verify that $\mathbf{A}$ is left nilpotent. We must show that ranges of idempotent 1-twins have the same cardinality. As in the first paragraph of the proof of Theorem 4.3, if $\mathbf{A}$ has idempotent 1-twins whose ranges have different cardinality, we may assume that they are $r_{\bar{a}}(x)=r(x, \bar{a})$ and $r_{\bar{b}}(x)=r(x, \bar{b})$ and that $r_{\bar{a}}(A) \subset r_{\bar{b}}(A)$. Choose $v \in r_{\bar{b}}(A)-r_{\bar{a}}(A)$ and let $u=r_{\bar{a}}(v)$. Then $u \neq v$ and

$$
\left[\begin{array}{ll}
r(u, \bar{a}) & r(u, \bar{b}) \\
r(v, \bar{a}) & r(v, \bar{b})
\end{array}\right]=\left[\begin{array}{ll}
u & u \\
u & v
\end{array}\right] \in M(1,1)
$$

so this can't happen. Therefore $\mathbf{A}$ is left nilpotent. Since $\mathbf{A}$ is regular, $\mathbf{H}(\mathbf{A})$ is left and right nilpotent.

Now we must show that $\mathbf{A}$ satisfies $[1,1]_{\delta} \stackrel{s s}{\sim} \delta$ for each $\delta$. As in the proof of Theorem 4.22 , if this fails it will fail for a strictly meet-irreducible $\delta$ with unique upper cover $\theta$ where $\operatorname{typ}(\delta, \theta)=\mathbf{2}$. In fact, as shown in the proof of that theorem, a failure of $[1,1]_{\delta} \stackrel{s s}{\sim} \delta$ implies the existence of an $n$, a polynomial $p^{\prime}(x, \bar{y}) \in \operatorname{Pol}_{n+1} \mathbf{A}, u, v \in A, \bar{a}, \bar{b} \in A^{n}$ and a $\langle\delta, \theta\rangle$ minimal set $U$ such that the following hold: $p^{\prime}\left(A, A^{n}\right) \subseteq U,\left.\mathbf{A}\right|_{U}$ has a Mal'cev polynomial $d(x, y, z)$ and

$$
p^{\prime}(u, \bar{a})=p^{\prime}(v, \bar{a})
$$

while

$$
p^{\prime}(u, \bar{b}) \theta-\delta p^{\prime}(v, \bar{b})
$$

If we let $p^{\prime \prime}(x, \bar{y})=d\left(p^{\prime}(x, \bar{y}), p^{\prime}(u, \bar{y}), p^{\prime}(u, \bar{b})\right)$, then

$$
\left[\begin{array}{ll}
p^{\prime \prime}(u, \bar{a}) & p^{\prime \prime}(u, \bar{b}) \\
p^{\prime \prime}(v, \bar{a}) & p^{\prime \prime}(v, \bar{b})
\end{array}\right]=\left[\begin{array}{ll}
p^{\prime}(u, \bar{b}) & p^{\prime}(u, \bar{b}) \\
p^{\prime}(u, \bar{b}) & p^{\prime}(v, \bar{b})
\end{array}\right] \in M(1,1)
$$

and $\left(p^{\prime}(u, \bar{b}), p^{\prime}(v, \bar{b})\right) \in \theta-\delta$. Thus, if $\mathbf{A}$ is weakly abelian, then $[1,1]_{\delta} \stackrel{s s}{\sim} \delta$ holds.

However, the class of finite weakly abelian algebras is not closed under the formation of homomorphic images. For example,

\begin{tabular}{|c||c|c|c|c|}
\hline $\mathrm{p}$ & $\mathrm{a}$ & $\mathrm{b}$ & $\mathrm{c}$ & $\mathrm{d}$ \\
\hline \hline $\mathrm{a}$ & $\mathrm{a}$ & $\mathrm{a}$ & $\mathrm{a}$ & $\mathrm{b}$ \\
\hline $\mathrm{b}$ & $\mathrm{a}$ & $\mathrm{a}$ & $\mathrm{a}$ & $\mathrm{b}$ \\
\hline $\mathrm{c}$ & $\mathrm{a}$ & $\mathrm{a}$ & $\mathrm{a}$ & $\mathrm{b}$ \\
\hline $\mathrm{d}$ & $\mathrm{b}$ & $\mathrm{b}$ & $\mathrm{b}$ & $\mathrm{c}$ \\
\hline
\end{tabular}


is an operation table for an abelian groupoid $\mathbf{A}$ where $\mathbf{A} / \operatorname{Cg}(a, b)$ is not even weakly abelian. So not every homomorphic image of a finite abelian algebra is weakly abelian. We do not know if the converse holds.

Problem 5 Is every finite weakly abelian algebra a homomorphic image of a finite abelian algebra?

We end with a final problem:

Problem 6 Characterize the finite weakly abelian algebras without referring to the weak term condition.

\section{References}

[1] P. Agliano and K. A. Kearnes, Congruence semimodular varieties II: locally finite varieties, to appear in the proceedings of the Jónsson Symposium, Algebra Universalis.

[2] R. Freese and R. McKenzie, Commutator Theory for Congruence Modular Varieties, LMS Lecture Notes No. 125, 1987.

[3] R. Freese, K. A. Kearnes and J. B. Nation, Congruences on conguence semidistributive algebras, to appear in the proceedings of the Birkhoff Symposium - 1991.

[4] D. Hobby and R. McKenzie, The Structure of Finite Algebras, AMS Contemporary Mathematics Series, 76, 1988.

[5] E.W. Kiss and P. Pröhle, Problems and results in tame congruence theory - A survey of the '88 Budapest Workshop, Preprint No. 60, Mathematical Institute of the Hungarian Academy of Sciences.

[6] R. McKenzie, G. McNulty and W. Taylor, Algebras, Lattices and Varieties, vol. 1, Wadsworth \& Brooks/Cole, 1987.

[7] R.D. Willard, $\mathbf{M}_{\mathbf{n}}$ as a 0,1-sublattice of Con A does not force the term condition, Proceedings of the AMS 104 (1988), 349-356.

1980 Mathematics subject classifications (1985 Revision ): Primary 08A05, 08A30.

Vanderbilt University, Nashville, TN 37235. 